\title{
The Spongtang Massif in Ladakh, NW Himalaya: An Early Cretaceous record of spontaneous, intra-oceanic subduction initiation in the Neotethys
}

\author{
Solomon Buckman ${ }^{\mathrm{a}, *}$, Jonathan C. Aitchison ${ }^{\mathrm{b}}$, Allen P. Nutman ${ }^{\mathrm{a}}$, Vickie C. Bennett ${ }^{\mathrm{c}}$, Wanchese M. Saktura ${ }^{\mathrm{a}}$, \\ Jessica M.J. Walsh ${ }^{\mathrm{a}}$, Sarah Kachovich ${ }^{\mathrm{b}}$, Hiroshi Hidaka ${ }^{\mathrm{d}}$ \\ a GeoQuEST Research Centre, School of Earth E Environmental Sciences, University of Wollongong, Australia \\ b School of Earth and Environmental Sciences, University of Queensland, Australia \\ ${ }^{c}$ Research School of Earth Sciences, Australian National University, Canberra, ACT 0200, Australia \\ d Department of Earth and Planetary Sciences, Nagoya University, Nagoya 464-8601, Japan
}

\section{A R T I C L E I N F O}

\section{Article history:}

Received 29 March 2018

Received in revised form 24 July 2018

Accepted 26 July 2018

Available online 29 July 2018

Handling Editor: M. Santosh

\section{Keywords:}

Himalaya

Spongtang ophiolite

Indus suture

India-Eurasia collision

Ladakh

\begin{abstract}
A B S T R A C T
The Spongtang Massif is a remnant of Neotethyan ocean crust emplaced onto the Indian passive margin along the Indus-Yarlung-Tsangpo Suture in the NW Himalayan region of Ladakh. The age, tectonic evolution and timing of ophiolite obduction are critical to our understanding of the mechanisms via which entire oceans are formed, consumed and partly preserved before the onset of terminal continent-continent collisions. Geochemistry of the gabbro and basaltic units suggest the presence of both MORB-type and primitive arc-related mafic rocks. Zircons extracted from the Spongtang Massif gabbros yield U-Pb (SHRIMP) ages of 136-133 Ma with initial $\varepsilon_{\mathrm{Hf}}$ values of +14 to +16 , indicating Early Cretaceous juvenile, depleted mantle sources devoid of contamination by older continental crust. Previously, Middle Jurassic ( $177 \mathrm{Ma})$ zircon ages were obtained from gabbro and we suggest these represent MORB-type Neotethyan oceanic crust through which a younger intra-oceanic islandarc (Spong arc) developed in response to subduction initiation during the Early Cretaceous ( 136 Ma). Our zircon ages are consistent with Early Cretaceous ages obtained for radiolarian cherts within the Spong Arc complex. Subduction beneath the Spong Arc continued until its collision with the northern Indian continental margin during the early Eocene. We suggest that the Spongtang Massif is equivalent to the nearby Dras island arc terrane. Intraoceanic subduction beneath this system was possibly initiated along NNE-SSW trending transform faults in the Neotethyan Ocean, along which different ages of ocean crust was juxtaposed, thereby development of the Early Cretaceous Spong Arc is superimposed on the older Jurassic Spongtang N-MORB crust. The juvenile $\varepsilon_{\mathrm{Hf}}$ signature indicates the subduction system that spawned the Spong island arc was not related to the coeval TransHimalayan (Ladakh-Gangdese) arc that developed along the southern margin of Eurasia. The age, composition and nature of geological relationships with the underlying Indian rocks indicate the Spong Arc was a juvenile, intra-oceanic terrane that first collided with India before the onset of final continent-continent collision. Therefore, final late Eocene Neotethys closure was between the Kohistan-Ladakh (Eurasian) continental arc and the already inactive Indian + Spongtang margin.
\end{abstract}

(c) 2018 Published by Elsevier B.V. on behalf of International Association for Gondwana Research.

\section{Contents}

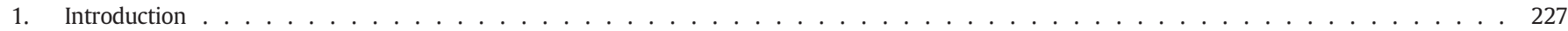

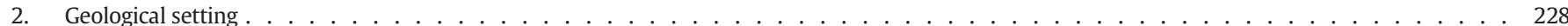

2.1. Spongtang Massif. . . . . . . . . . . . . . . . . . . . . . . . . . . . . . . . 228

2.2. Indian margin -Zanskar Group ． . . . . . . . . . . . . . . . . . . . . . . . . . . . . . . . . . . . 229

2.3. Dras Volcanics and Nindam Formation . . . . . . . . . . . . . . . . . . . . . . . . . . . . . . . . . . . . . . 2229

2.4. Chilling Formation . . . . . . . . . . . . . . . . . . . . . . . . . . . . . 229

\footnotetext{
* Corresponding author.

E-mail address: solomon@uow.edu.au (S. Buckman).
} 
2.5. Ladakh Batholith (Trans-Himalayan Batholith)

Tar Group - Ladakh Fore-arc Basin.

2.7. Post-collisional Indus Group (molasse)． . . . . . . . . . . . . . . . . . . . . . . . . . . . . . . . . . . . . 232

3. Analytical methods . . . . . . . . . . . . . . . . . . . . . . . . . . . . . . . . . 232

$4 . \quad$ Results . . . . . . . . . . . . . . . . . . . . . . . . . . . . . . . . 232

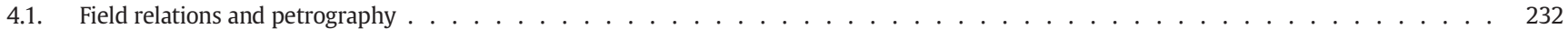

4.1.1. Gabbro . . . . . . . . . . . . . . . . . . . . . . . . . . . . 232

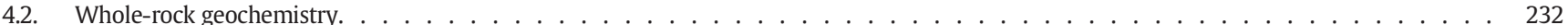

4.3. SHRIMP U-Pb zircon results . . . . . . . . . . . . . . . . . . . . . . . . . . . . . . . . . . . 237

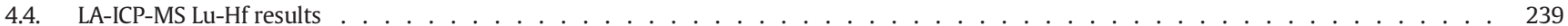

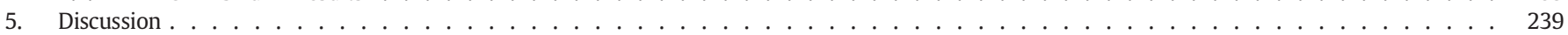

5.1. Geochronology and $\mathrm{Hf}$ isotope signatures. . . . . . . . . . . . . . . . . . . . . . . . . . 239

5.2. Intra-oceanic geodynamic setting of the Spong Arc . . . . . . . . . . . . . . . . . . . . . . . . . . . . . . 241

5.3. Spong and Dras island $\operatorname{arcs} \ldots \ldots \ldots \ldots \ldots \ldots$

5.4. Timing of ophiolite-arc collision with India and closure of the Neotethys . . . . . . . . . . . . . . . . . . . . . . . . . . . 241

5.5. Paleogeography and subduction initiation within Neotethys . . . . . . . . . . . . . . . . . . . . . . . . 243

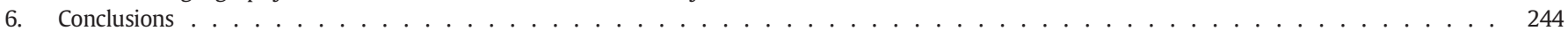

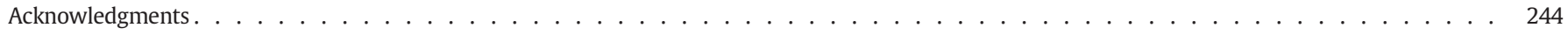

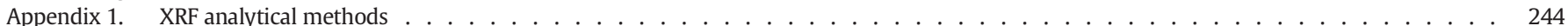

Appendix 2. SHRIMP U-Pb analytical method and data appraisal . . . . . . . . . . . . . . . . . . . . . . . . . . . . . . . . . . . . . . . . . . . 244

Appendix $3 . \quad$ LA-MC-ICP-MS zircon Lu-Hf isotopes . . . . . . . . . . . . . . . . . . . . . . . . . . . . . . . . . . . . . . 244

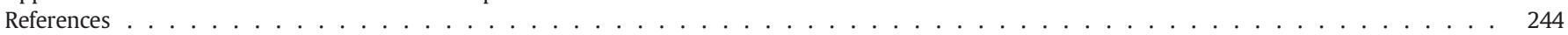

\section{Introduction}

Debates about the age and nature of the Himalayan Orogeny centre on whether the collision was purely a single continent-continent event involving the Eurasian active margin and the Indian passive margin - see discussion by Hu et al. (2016), or involved intra-oceanic ophiolite and island-arc terranes in multiple collisions with either India or Eurasia before the onset of terminal continental collision - see discussions by Aitchison et al. (2007a). Despite the attractive simplicity of a single continental collision model (Najman et al., 2017; Wang et al., 2017), there is mounting evidence that the Indus-Yarlung-Tsangpo Suture (IYTS) extending from Tibet in the east to Pakistan in the west contains preserved fragments of juvenile ophiolite and island arc complexes that developed within a Tethyan intra-oceanic setting far from the influence of any continental crust (Aitchison et al., 2000; Corfield et al., 2001; Hébert et al., 2012; McDermid et al., 2002; Robertson and Degnan, 1994). The IYTS ophiolites typically occur as nappes thrust over the Indian margin or are disrupted into mélange within fault zones against the Eurasian fore-arc basin rocks. Importantly, the contact with Eurasian rocks is always faulted and never stratigraphically conformable (Aitchison et al., 2003) indicating they are allochthonous terranes. Paleomagnetic evidence indicate that the ophiolites formed at equatorial latitudes (Abrajevitch et al., 2005) somewhere between 1000 and $2500 \mathrm{~km}$ south of the active southern margin of Eurasia which lay between 15 and $25^{\circ} \mathrm{N}$ during Early Cretaceous time (Abrajevitch et al., 2005; Klootwijk et al., 1979; Yang et al., 2015).

The Spongtang Massif in the Zanskar mountains of Ladakh is one of the most complete fragments of Neotethyan Ocean crust preserved along the IYTS of the western Indian Himalaya (Fuchs, 1982; Gansser, 1964; Reuber, 1986; Searle, 1986). The Spong island-arc complex intruded and developed on top of this ophiolite (Pedersen et al., 2001) and was initially regarded as equivalent to the Dras Volcanics and sedimentary equivalent Nindam Formation to the north (Fuchs, 1982). Together the ophiolite and island arc represent an important record of intra-oceanic processes operating in the Neotethyan Ocean before India collided with Eurasia to form the Himalaya (Aitchison et al., 2007a; Corfield et al., 2001). The ophiolite-arc complex collided with the northward migrating Indian continental margin at a north-dipping subduction zone resulting in emplacement of an ophiolite-arc thrust sheet onto the shallow marine, passive margin sequence - the Zanskar Supergroup (Reuber et al., 1992). This event is widely interpreted as having occurred before final closure of the Neotethyan Ocean and subsequent onset of final continent-continent collision (Aitchison et al., 2007a; Corfield et al., 2001; Pedersen et al., 2001; Reuber et al., 2015).
However, uncertainties remain regarding the age, paleogeography, tectonic setting and timing of emplacement of the Spongtang Massif. Previous $\mathrm{U}-\mathrm{Pb}$ radiometric ages are limited to $\mathrm{U}-\mathrm{Pb}$ zircon ages of a diorite yielding $177 \pm 1 \mathrm{Ma}$, and an andesite $88 \pm 5 \mathrm{Ma}$, where the latter is associated with the Spong arc sequence overlying the ophiolite. Pedersen et al. (2001) interpreted the older age to represent that of the Neotethyan ocean crust on top of which the Spong arc developed, while the Late Cretaceous andesite age of $\sim 88$ Ma represents the minimum age of subduction initiation to form the Spong island-arc complex. Numerous $\mathrm{K}-\mathrm{Ar}$ dates by Reuber (1989) revealed two clusters of amphibole ages of 140-125 Ma and $170 \mathrm{Ma}$ (Fig. 10) which approximately fits with the zircon ages, and again are consistent with a Jurassic MORB ophiolite age and a subsequent Cretaceous period of island arc igneous activity. Biostratigraphic investigations by Baxter et al. (2010) documented Early Cretaceous radiolarian faunal assemblages from red cherts amongst the Spong Arc volcanic complex, which correlates well with ophiolites along strike at Nidar, Xigaze and Zedong. Colchen et al. (1987) reported Eocene radiolarians from chert blocks in footwall mélange within the thrust zone below the ophiolite that appear to represent the youngest deep marine units of the NW Indian passive margin sequence.

Therefore, the Spongtang Massif is a critical piece of this complex tectonic puzzle. Interpretations of the tectonic setting in which it might have formed include; A) slivers of the Neotethyan Ocean accreted beneath the fore-arc of the Ladakh Arc before it was obducted onto the Indian margin (Steck, 2003); B) fore-arc basement of the Dras Arc, which evolved into the Ladakh-Kohistan Arc before final continental collision involving only a single north-dipping subduction zone (Fuchs, 1982); C) a suprasubduction zone ophiolite that evolved into the Spong Arc at the southernmost of two subduction zones between India and Asia that was partially subducted beneath the Sapi-Shergol accretionary complex of the Dras Arc before colliding with India (Groppo et al., 2016; Mahéo et al., 2006); D) oceanic basement to the intra-oceanic Spong Arc, which developed above the southernmost of three north-dipping subduction zones between India and Eurasia and collided first with India at $70 \mathrm{Ma}$, while the Dras-Kohistan Arc collided with the TransHimalayan Arc between 102 and 75 Ma before it evolved into the continental Ladakh (Trans-Himalayan) Arc prior to final continental collision at $55 \mathrm{Ma}$ (Corfield et al., 2001). We address aspects of the age and origin of the Spongtang Massif using an integrated program of field geology, whole-rock geochemistry and $\mathrm{U}-\mathrm{Pb}$ and $\mathrm{Lu} / \mathrm{Hf}$ analysis of zircons extracted from gabbro and leucogabbro samples, to assess the influence any continental crustal material had during generation of melts during ophiolite-arc formation. 


\section{Geological setting}

\subsection{Spongtang Massif}

The Spongtang Massif crops out as an isolated klippe situated between 4000 and $6000 \mathrm{~m}$ elevation near the village of Photoksar about $30 \mathrm{~km}$ south of the Indus Suture (Fig. 2). This ophiolite has attracted the attention of geologists for over a century (La Touche, 1888; Lydekker, 1880; Lydekker, 1883; MacMahon, 1901) and was the focus of several mapping expeditions in the 1980s, which provided detailed geological maps (Bassoullet et al., 1980; Colchen et al., 1987; Fuchs, 1979, 1982; Honegger et al., 1982; Kelemen and Sonnenfeld, 1983; Reibel and Reuber, 1982; Reuber, 1986; Reuber et al., 1992; Reuber et al., 2015; Reuber et al., 1989).

The Spongtang Massif displays all elements of a formerly complete "Penrose-style" ophiolite stratigraphy, albeit significantly disrupted by faulting. Early studies concentrated on the mantle peridotites and lower crustal cumulates (Reibel and Reuber, 1982) in an attempt to identify fabrics that might indicate stress regimes associated with a spreading ridge (Reuber, 1986). Detailed mapping identified an upper lherzolite unit rich in pyroxene structurally overlying a more depleted harzburgite unit (Reuber et al., 1992). Lower crustal cumulate rocks include wehrlite, pyroxenite and gabbro. They crop out along the eastern side of the Photang valley and west side of Marling chu valley (Fig. 2), where they are intruded by medium-grained basaltic dykes (Corfield et al., 2001; Reuber et al., 1992; Reuber et al., 2015). Minor gabbro cumulates occur within the lower unit on the east side of Photang Kangri and along the Marling Valley section (Reuber et al., 1992). Gabbro also occurs within the dominantly basaltic-andesite volcanic west side of
Photang Kangri and along Photoksar Valley where our samples were collected. Corfield et al. (2001) observed two generations of dykes; 1) coarse-grained pegmatitic gabbroic dykes intruding the harzburgites, and 2) medium-grained basaltic dykes indicating at least two stages of mantle melt generation. Dunite bodies also intrude both peridotite units. Corfield et al. (2001) reported rare plagiogranites within the mantle sequence but were not successful in extracting zircons from these samples. We collected gabbro, leucogabbro and pegmatitic gabbro from the Photong Valley for zircon extraction. Two samples gave low yields of small zircons amenable to dating using the Sensitive HighResolution Ion Microprobe (SHRIMP) at Hiroshima University.

Partial remnants of a once extensive ophiolite thrust sheet over the northern Indian margin are locally preserved along the Indus-YarlungTsangpo Suture Zone. The nearest complete ophiolite sequence to Spongtang Massif is the Nidar ophiolite ( 140 Ma) about $120 \mathrm{~km}$ east (Ahmad et al., 2008; Mahéo et al., 2004; Zyabrev et al., 2008). Further east, the Indus Suture is offset dextrally by the Karakorum Fault but correlatives are found in Tibet as part of the Dazhuqu and Zedong terranes (Aitchison et al., 2000). Individually, these include ophiolites recorded at Jungbwa (Miller et al., 2003), Saga and Sangsang (Bédard et al., 2009), Xigaze (Dubois-Cote et al., 2005; Dupuis et al., 2005; Girardeau et al., 1984; Girardeau et al., 1985a; Nicolas et al., 1981; Wang et al., 1987), Dazhuqu (Girardeau et al., 1985b; Xia et al., 2003), Zedong (Aitchison et al., 2007b; McDermid et al., 2002) and Luobusa (Zhou et al., 1996). Correlatives to the west in Pakistan include Bela (Sarwar, 1992; Zaigham and Mallick, 2000), Muslim Bagh (Moores et al., 1980) and Waziristan ophiolites (Jan et al., 1985). The magmatic ages of these ophiolites vary from Jurassic to Cretaceous (Fig. 1).

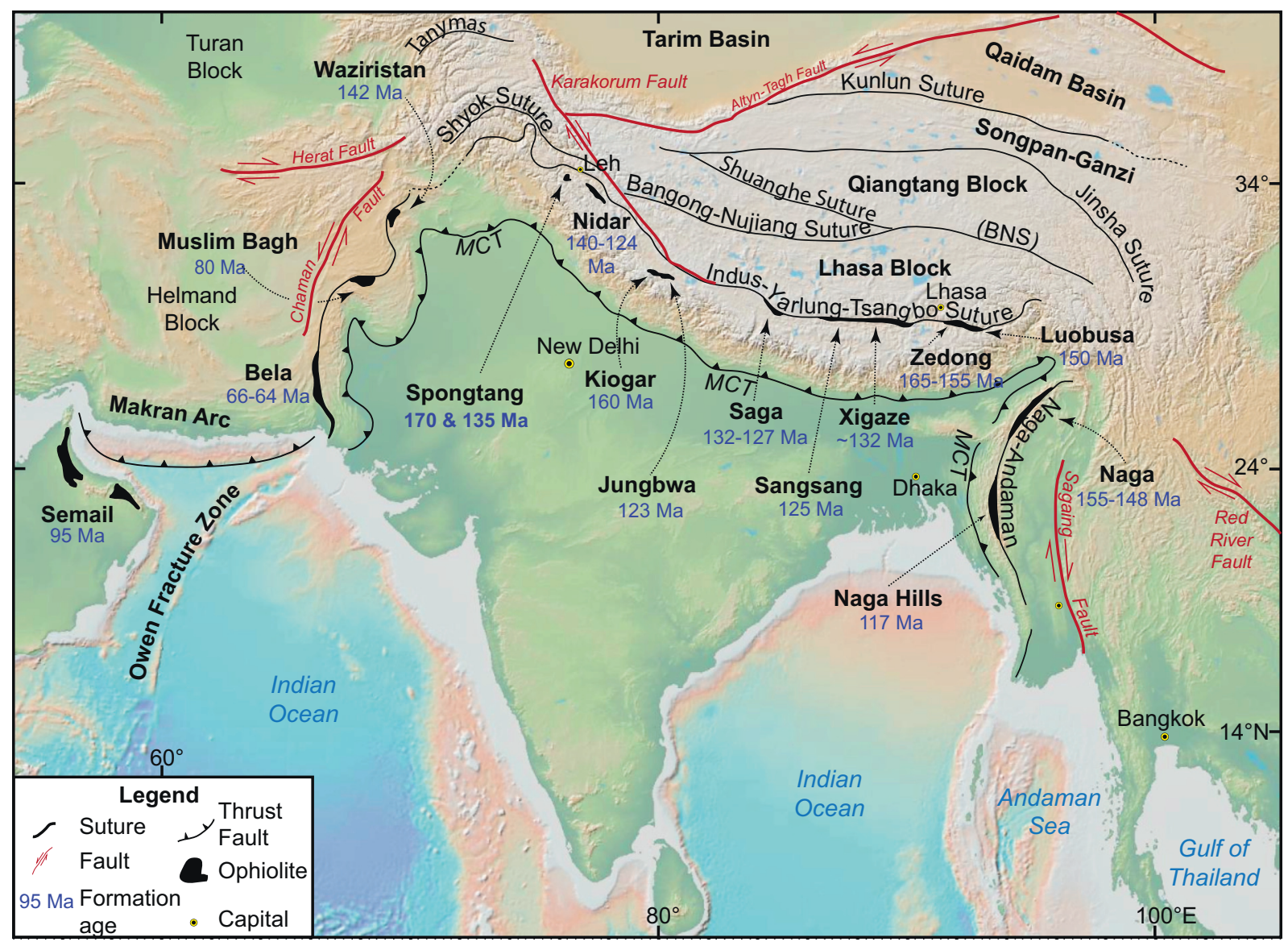

Fig. 1. Regional tectonic setting of the Himalaya showing Tethyan ophiolite occurrences and their age of formation (blue). Basemap sourced from GeoMapApp software (Ryan et al., 2009). 


\subsection{Indian margin - Zanskar Group}

The Permian-Eocene northern Indian passive margin platform sequence or "Zanskar Supergroup" over which the ophiolite klippe is thrust has been described in detail previously (Fuchs, 1982; Gaetani and Garzanti, 1991; Garzanti et al., 1987). Mud-matrix mélange directly underlies the ophiolite klippe (Reuber et al., 1992; Reuber et al., 2015). Collectively, the large and varied blocks within a mud-matrix mélange are associated with the Lamayaru Complex (Brookfield and AndrewsSpeed, 1984). Robertson and Degnan (1993) interpret the Lamayuru Complex as outer shelf to slope deposits of the Indian margin. This margin experienced pulses of extension and drastic collapse of the carbonate platform edge during the mid-Jurassic as a convergent plate boundary approached resulting in submarine channels being filled with a mixture of shallow water limestone olistoliths and deep marine cherts and clastics. While the Lamayaru Complex was initially deposited as a passive margin succession, it displays scaly mud-matrix and block in matrix textures characteristic of overpressuring due to tectonic crustal loading. Mud-matrix mélange is commonly associated with collision complexes involving obduction of an island-arc complex onto a passive continental margin, for example, the Lichi mélange in Taiwan (Huang et al., 2008). Such mélange within the Lamayaru Complex is possibly a correlative of the Yamdrok mélange in Tibet (Liu and Aitchison, 2002).

The youngest marine shales deposited on the Indian passive margin are the Lower Eocene Chulung la Formation and Kong slates which structurally underlie the Spongtang Massif thrust sheet further south at Dibling (Fuchs, 1982; Fuchs and Willems, 1990). Najman et al. (2017) undertook detrital zircon studies of the Kong and Chulung la formations and reported a youngest detrital zircon age of $\sim 53$ Ma thereby establishing the maximum depositional age. Both formations show a strong Mesozoic to Cenozoic distribution (55-70 Ma and 90-100 Ma) and rare Precambrian zircons. Najman et al. (2017) interpret zircon grains older than $\sim 130$ Ma to have been sourced from southern Eurasian margin because of the presence of two $140 \mathrm{Ma}$ grains of positive $\varepsilon \mathrm{Hf}$ value while older Precambrian grains are display mostly negative $\varepsilon_{\mathrm{Hf}}$ values and therefore sourced from (Gondwanan) Lhasa terrane. Zircons $<115$ Ma display mostly positive $\varepsilon_{\mathrm{Hf}}$ values that drop to negative values for the youngest grains which they suggest is consistent with a Kohistan-Ladakh-Gangdese Arc source. However, we suggest that the age and $\varepsilon_{\mathrm{Hf}}$ values of the Kong and Chulung la formations are consistent with derivation from the structurally overlying Spong and Dras island arc immediately overlying and to the north of these units. The intra-oceanic Spong and Dras island arc would have been active until its collision with India during the early Eocene. The trend towards negative $\varepsilon_{\mathrm{Hf}}$ values in younger grains is consistent with subduction of continental margin sediment from India beneath the Dras Arc just prior to arc-continent collision along with an influx of Indian-derived older Precambrian zircons. The two 140 Ma zircons with positive $\varepsilon \mathrm{Hf}$ values are consistent with zircons from the Spongtang Massif presented here.

Reuber et al. (2015) suggested that the youngest sediments of the Indian passive margin sequence must constrain the maximum age of obduction and therefore structural relationships between the lower Eocene Chulung la Formation and Kong Slates that tectonically underlie the Spongtang Massif constrain ophiolite obduction to post- early Eocene. This is consistent with tectonic reconstructions that place northern India at about $10^{\circ} \mathrm{N}$ (Aitchison et al., 2007a; Dewey et al., 1989; Molnar and Tapponnier, 1975) and at least $1000 \mathrm{~km}$ south of Eurasia at $45 \mathrm{Ma}$ when the Ladakh Arc on the southern margin of Eurasia was at $23^{\circ} \mathrm{N}$ (Klootwijk et al., 1979). However, Corfield et al. (1999) proposed that obduction of the Spongtang Massif must have occurred during the Late Cretaceous based on their interpretation that an allochthonous thrust sheet of continental slope deposits is truncated by uppermost Cretaceous to lower Eocene deposits and are therefore post-collisional. They invoke a later phase of thrust faulting following collision of India with Eurasia to explain why the Spongtang Massif is thrust over these lower Eocene deposits.

\subsection{Dras Volcanics and Nindam Formation}

The Dras Volcanics and correlative volcaniclastic rocks of the Nindam Formation crop out extensively to the east of Kargil and represent a poorly understood package of island-arc volcanic rocks and immature volcaniclastic rocks faulted between Eurasian margin derived sediments (Tar and Indus groups) to the north and the Indian margin (Zanskar Group) to the south (Clift et al., 2000; Fuchs, 1982; Reuber, 1989; Robertson and Degnan, 1994). They were originally described and referred to as the Zanskar flysch by Sterne (1979), which is overlain by the Chilling Formation (Fuchs, 1986). Some interpret the Nindam Formation as a continuation of the Indus Molasse sequence (Tar Group) suggesting that together they formed in a fore-arc basin that flanked the Ladakh Arc to the north (Garzanti and Van Haver, 1988; Henderson et al., 2010; Henderson et al., 2011). However, contacts between the two units are faulted and locally marked by extensive mélange development, such as the Mongyu mélange located between Khaltse and Mongyu villages (Fuchs, 1982). Detailed petrographic and lithofacies analysis of the Dras-Nindam units by Robertson and Degnan (1994) indicates that the Nindam Formation differs markedly from the Andean-type fore-arc succession of the Trans-Himalaya (Ladakh Block) or Tar Group immediately to the north of the Mongyu mélange (Fig. 2). Sandstones from the Albian-Aptian Khalsi limestone (Tar Group) north of the Mongyu mélange are dominated by metamorphic quartz and minerals such as hornblende that are rare or absent in the coeval Nindam Formation. The Paleocene Trans-Himalaya fore-arc basin succession overlying the Khalsi Limestone contains thick, channelized conglomerate units containing abundant granite/rhyolite clasts while coeval sections of the Nindam Formation formed in deep marine, hemipelagic environments and are dominated by immature volcaniclastic sedimentary and pyroclastic rocks. On the basis of these fundamental differences, Robertson and Degnan (1994) interpret the Dras Volcanics and Nindam Formation to have formed in an intraoceanic island-arc setting, most likely in a fore-arc basin setting assuming northward subduction. Differences between the Dras and Ladakh arcs are further highlighted by distinct geochemical differences of volcanic rocks of the island arc Dras Volcanics and continental arc Khardung Volcanics respectively as reported by Clift et al. (2002a).

\subsection{Chilling Formation}

The Chilling Formation was first described and named by Sterne (1979) as a sequence of red-purple-green siltstones and mass-flow volcaniclastic conglomerates (Skiu conglomerate) that overlie the Dras-Nindam unit (Zanskar flysch). Clasts within conglomerates of the Chilling Formation consist of ophiolite-derived peridotite, gabbro, basalt, chert and volcaniclastic rocks similar to lithologies within the adjacent Dras ophiolitic mélange. They also include nummulitic limestone and quartzite clasts derived from the Indian Zanskar Group and Lamayaru Complex. Sterne (1979) considered the Chilling Formation to be an outlier equivalent to the Spongtang Massif but Fuchs (1986) renamed it the "Chilling Molasse" and interpreted it as the youngest portion of the Dras unit. Brookfield and Andrews-Speed (1984) were unable to correlate this unit and regarded it as unassigned molasse. Searle et al. (1990b) regarded this unit as equivalent to both the Chogdo and Nindam formations, while others assigned it to the Chogdo and Khalsi formations (Clift et al., 2001, 2002a, 2002b). Henderson et al. (2011) disagreed with previous correlations between the Chilling and Chogdo formations based on the radically different geochemical and detrital zircon signatures of these units. More recently, Baxter et al. (2016) presented chrome spinel evidence from the Chilling Formation and concluded that the ultramafic clasts present were derived from the Spongtang Massif. The Chilling Formation consists entirely of ophiolite 


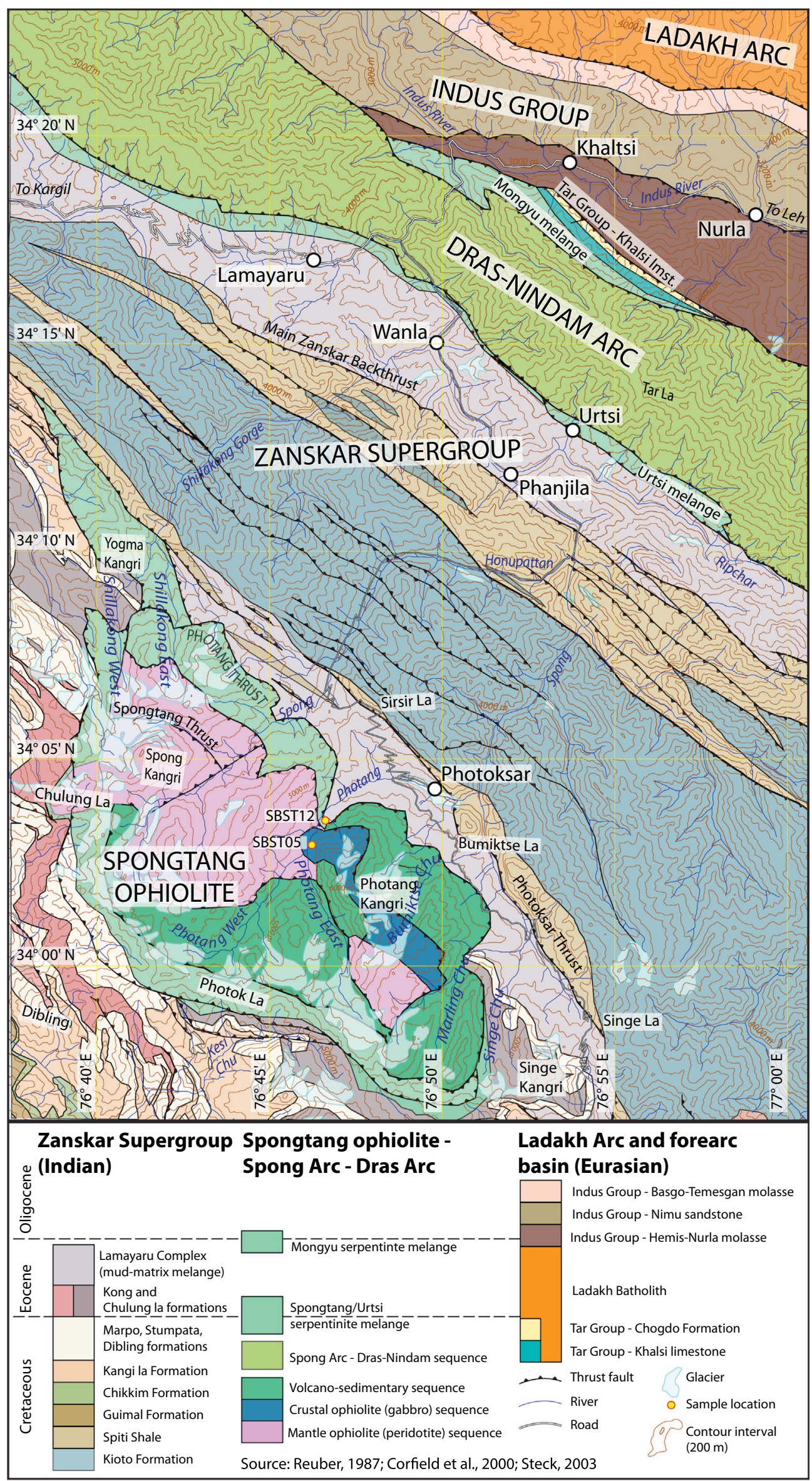


and Indian-derived detritus and this is reflected in detrital zircon populations which show little or no Eurasian source. The youngest reported zircon population is 159 Ma which Henderson et al. (2011) suggest is derived from the Indus ophiolites such as Spongtang. In contrast, the Chogdo Formation is dominated by $40-85$ Ma zircons derived from the Ladakh Arc. Chilling Formation shales have high $\mathrm{Cr}$ and Ni concentrations approaching values within the ophiolite source rocks while Chogdo Formation has low concentrations of $\mathrm{Cr}$ and $\mathrm{Ni}$ and is dominated by felsic volcanic and intrusive clasts. Finally, the red conglomerates and shales typical of the Chilling Formation are not present in the Chogdo Formation and on the weight of this evidence Henderson et al. (2011) preferred not to correlate the two formations.

In Tibet, obduction of the ophiolite-arc complexes with the Indian margin is marked by the presence of a distinct conglomerate unit the Luiqu conglomerate (Davis et al., 2004) which is probably equivalent to the Chilling Formation in Ladakh (Baxter et al., 2016). Deposition of this distinct ophiolite + Indian continent derived conglomerate marks the onset of arc-continent collision at $255 \mathrm{Ma}$. The final continent-continent collision is marked by the deposition of the Lower Miocene ( $34 \mathrm{Ma}$ ) Gangrinboche conglomerates in Tibet (Aitchison et al., 2002) and the Indus Group (molasse) in Ladakh (Henderson et al., 2010).

\subsection{Ladakh Batholith (Trans-Himalayan Batholith)}

The Ladakh Batholith occurs as a WNW-ESE trending linear belt, spanning an area approximately $600 \mathrm{~km}$ long and $30-80 \mathrm{~km}$ wide (Singh et al., 2007) and is bound by the Indus Suture Zone to the south and the Shyok Suture Zone along its northern margin (Frank, 1977). The Ladakh Arc is composed of a calc-alkaline intrusive suite, ranging in composition from gabbroic to granitic (Honegger et al., 1982; Singh et al., 2007), and a carapace of andesitic to rhyolitic eruptive rocks assigned to the Khardung Volcanics (Dunlap and Wysoczanski, 2002). Leucogranite intrusions (Reichardt et al., 2010) and andesitic dykes (Heri et al., 2015) are also reported. Locally, the Ladakh Batholith intrudes the Jurassic?-Cretaceous Shyok Volcanics (Borneman et al., 2015) along its northern margin, evident by the numerous basaltic xenoliths within granitoids (Kumar et al., 2016) and minor Jurassic sedimentary rocks of the Tsoltak Formation (Ehiro et al., 2007; Reuber, 1990). Vadlamani and Guha (2002) reported Miocene ( $24 \mathrm{Ma})$ ultrapotassic dykes that intruded the Ladakh Arc and correlated these with post-collisional intrusions in Tibet (Miller et al., 1999; Ravikant, 2006).

The Ladakh Batholith, together with the Kohistan Batholith to the north-west and the Gangdese Batholith to the east, collectively forms the Trans-Himalaya Batholith, which has yielded diachronous magmatic crystallization ages between $103 \mathrm{Ma}$ to $41 \mathrm{Ma}$, younging to the east (Ravikant et al., 2009; Reichardt et al., 2010; Shellnutt et al., 2014; Weinberg and Dunlap, 2000). The Gangdese Batholith displays discrete pulses of magmatism at $205-152, \sim 109-80, \sim 65-41$ Ma (Ji et al., 2009) while the Ladakh Batholith displays distinct magmatic pulses between 66-41 and 85-75 Ma (White et al., 2011) and a smaller 90-110 Ma peak recorded in detrital zircon studies of the Indus Group (Henderson et al., 2010) but no evidence of older pulses. Alternatively, Bouilhol et al. (2013) suggest that the Ladakh-Kohistan Batholith is not part of the Trans-Himalayan Batholith but a separate intraoceanic island arc generated on a separate subduction system to the continuous Gangdese-Karakoram continental arc developing on the southern margin of Eurasia. They include the Spongtang Massif and Dras Arc as different sections of a single arc that includes the Kohistan-Ladakh batholith. While the Kohistan-Ladakh batholiths do display island arc characteristics it is difficult to reconcile the need to separate them from the Gandese Batholith which has the same age range and juvenile $\varepsilon_{\mathrm{Hf}}$ values and is continuous along strike when displacement across the Karakoram Fault $(\sim 150 \mathrm{~km})$ is taken into consideration. The Karakoram Batholith displays more similarities in age and composition (Rex et al., 1988; Searle et al., 1990a; Searle and Tirrul, 1991; Searle et al., 1998) with Mesozoic granites along the Bangong-Nujiang Suture between Qiangtang and Lhasa blocks in Tibet (Liu et al., 2014). Rolland (2002) suggested that the relationship between the Kohistan, Ladakh and Gangdese batholiths might be transitional from purely island arc in the west to continental arc in the east and we suspect it was similar to the modern day Aleutian Arc. In Tibet, the youngest age of the equivalent Gangdese Batholith is $41 \mathrm{Ma}$ (Ji et al., 2009) but detrital zircon studies indicate that magmatic activity may be as young as $37 \mathrm{Ma}$ (Aitchison et al., 2011), suggesting that northward subduction beneath the southern margin of Eurasia (Lhasa Block) continued until late Eocene time and continent-continent collision may not have started until at least after $\sim 37 \mathrm{Ma}$.

\subsection{Tar Group - Ladakh Fore-arc Basin}

The oldest units within the Indus Basin are the Albian-lower Eocene predominantly marine sequence commonly referred to as the Tar Group (Henderson et al., 2010; Searle et al., 1990b; Sinclair and Jaffey, 2001) or Indus Flysch (Fuchs, 1979, 1981). The Tar Group consists of deep marine limestones and turbidites (Khalsi limestone), overlain by Late Cretaceous to Paleocene marine marls, mass flow deposits and shales of the Jurutze Formation. The Tar Group is interpreted as the marine fore-arc basin to the Ladakh Arc, which developed slightly outboard of southern Eurasia prior to the onset of continental collision (Clift et al., 2002b; Garzanti and Van Haver, 1988; Sinclair and Jaffey, 2001; Steck et al., 1993). In Tibet, this fore-arc sequence is well recognized as the Xigaze Group (Aitchison et al., 2011; Dürr, 1996; Einsele et al., 1994; Wang et al., 1999). Henderson et al. (2010) collectively refer to the Tar Group and the conformably overlying, post-early Eocene Indus Group as Indus Basin sedimentary rocks.

The basal contact of the Tar Group has been interpreted differently by different authors. Searle et al. (1990b) considered the oldest units of the Tar Group as disconformable cover to the Cretaceous Nindam Formation. However, our geological mapping indicates that the oldest unit in the Tar Group, the Khaltsi Limestone, is always in fault contact with the Dras-Nindam unit to the south with the contact marked by distinctive serpentinite-matrix mélange (Mongyu mélange). This melange includes blocks of peridotite, gabbro, basalt and volcaniclastic rocks. Clift et al. (2000) assigned the lowermost parts of the Indus Group to the Chogdo Formation and interpreted this unit as a correlative of the Chilling Formation, which they considered, unconformably overlies the Nindam Formation, Lamayaru Complex and Tar Group and contains ophiolitic clasts. This led Clift et al. (2002b) to infer that the age of the Chogdo Formation might constrain the maximum age of collision. Based on this reasoning, they suggested that the well-established Early Eocene (Ypresian) age of the Nummulitic limestone, which overlies the Chogdo Formation, indicates continent-continent collision must have occurred prior to 49 Ma. However, more detailed stratigraphic and provenance studies by Henderson et al. (2011) exclude any possibility that the Chogdo and Chilling formations might be correlatives thus they cannot be used to constrain the timing of collision. The Chogdo Formation is dominated by felsic igneous clasts derived from the Ladakh Arc whereas the Chilling Formation is dominated by ophiolitic and quartzite-limestone clasts of Indian and ophiolite provenance but no Eurasian influence. The Chilling Formation formed on the Indian Plate as Indus ophiolites were being obducted, whereas the Chogdo Formation accumulated in a fore-arc basin associated with the Ladakh Arc on the southern Eurasian margin. 


\subsection{Post-collisional Indus Group (molasse)}

Fore-arc basin sedimentary rocks of the Tar Group are succeeded by post-early Eocene to Miocene molasse of the Indus Group (Baud et al., 1982; Henderson et al., 2010; Searle et al., 1990b; Sinclair and Jaffey, 2001; Tewari, 1964). These high-energy, fluvial conglomerates and sandstones mark the onset of the India-Eurasia continental collision and intra-montane basin development. In places, the Indus Group is deposited unconformably on granites of the Ladakh Batholith (Garzanti and Van Haver, 1988; Searle et al., 1997) indicating considerable uplift and erosion of the Ladakh Arc prior to deposition. Detrital zircon studies of the Indus Group by Henderson et al. (2011) reveals that the youngest detrital zircon population is $41 \mathrm{Ma}$ within sandstones of the upper Nimu Formation suggesting that calc-alkaline magmatism within the Ladakh Arc continued until at least $41 \mathrm{Ma}$. This is significant because the age of the youngest arc-related, calc-alkaline magmatic rocks possibly constrains the timing of terminal continental collision (Searle et al., 1988). In Tibet, detrital, arc-derived zircons as young as $37 \mathrm{Ma}$ from the upper Oligocene post-collisional Gangrinboche conglomerates (Aitchison et al., 2011) indicate that subduction-related convergent margin magmatism continued along the southern margin of Eurasia until at least the Late Eocene before final collision of India and Eurasia. The Indus Group sedimentary rocks have experienced substantial NESW directed compression resulting in $\mathrm{km}$-scale folds and some refolded folds (Henderson et al., 2010; Searle et al., 1990b). This intense deformation and faulting complicates resolution of stratigraphic relations in Ladakh.

\section{Analytical methods}

Standard methods were employed for whole-rock geochemistry including X-ray fluorescence (XRF) for major and trace elements at University of Wollongong (Table 1 ) and inductively coupled plasma mass spectrometry (ICP-MS) for rare-earth elements (REE) at Australian Laboratory Services in Brisbane (Table 1). Methods are described in detail in Appendix 1. U-Pb zircon geochronology (Table 2) was undertaken using the SHRIMP at Hiroshima University and detailed methodology is given in Appendix 2. Zircon Lu-Hf isotope analysis (Table 3) was undertaken using LA-ICP-MS at the Australian National University (ANU) (Appendix 3). Method description along with the complete data for the 6 reference zircons (FC-1 Plesovic, QGNG, Monastery, Mud Tank and R-33) analyzed throughout the analytical session is provided in Appendix 3.

\section{Results}

\subsection{Field relations and petrography}

\subsubsection{Gabbro}

Gabbros collected from Photang valley range in composition and texture from layered olivine-hornblende gabbro (SBST19 - Figs. 3 and 4) to isotropic hornblende gabbro, leucogabbro and pegmatitic hornblende gabbro dykes (Fig. 4). The amphibole-rich gabbro-diorite dykes intrude both the mantle (ultramafic) and mafic volcanic sequences. Leucocratic gabbros are also incorporated as blocks in the basal serpentinite mélange. Leucogabbro sample SBST05 was collected from an outcrop near the base of the klippe along Photoksar valley. The leucogabbro intrudes a larger serpentinised peridotite block and is incorporated into the highly disrupted, basal serpentinite-matrix mélange (Fig. 3). Other samples for this study were collected from the upper reaches of the Photang valley (Fig. 2). Thin (1-3 m) dykes and sills of pegmatitic, amphibole-rich gabbro intrude the massive gabbro and peridotites (Fig. 3). The abundance of amphibole as part of the igneous assemblage in these rocks indicates a hydrous magma.

Thin-section petrography of gabbro samples shows that unaltered, euhedral hornblende phenocrysts are the dominant mineral phase in most samples including the more mafic olivine-hornblende gabbro (SBST19 - Fig. 4A). Igneous hornblende occurs as large euhedral phenocrysts aligned with cumulate layering and displays strong green to light brown pleochroism typical of magmatic hornblende. Isotropic gabbros contain randomly orientated hornblende, pyroxene and plagioclase phenocrysts. Some leucogabbro samples contain kaersutite amphibole identified by its deep yellow/orange to brown pleochroism. The occurrence of rare poikilitic olivine within clinopyroxene phenocrysts is interpreted to demonstrate an early stage of anhydrous, tholeiitic crystallization prior to water saturation (Fig. 4A). Clinopyroxene is present in the more mafic gabbros but is always subordinate to hornblende and commonly rimmed by hornblende. Early stage, subhedral plagioclase phenocrysts are present in most samples but are ubiquitously altered to a semi-opaque, yellow-brown mixture of fine-grained albitesericite-epidote-calcite-quartz (Fig. 4A-H). Accessory igneous minerals include late stage magnetite rimming hornblende, rutile, quartz, ilmenite, zircon, and apatite. Some of the more felsic leucogabbros contain needles of metamorphic actinolite (Fig. 4F) rimming the igneous hornblende. Selective alteration of plagioclase is pronounced such that Carlsbad twinning is rarely observed and distinctly contrasts with neighbouring, unaltered hornblende and clinopyroxene (Fig. 4D). This suggests that crystallization initially followed a typical anhydrous path of olivine-anorthite-clinopyroxene before water saturation of the magma resulted in hornblende crystallization and pervasive alteration of earlier crystallized anorthite. Alternatively, the original anhydrous gabbro may have undergone re-melting at a later stage to generate a hydrous hornblende gabbro. Small lenses and veins of fresh, interlocking quartz, albite and microcline occur in leucogabbro sample SBST16 (Fig. 4D-F) and in the pegmatitic gabbro sample SBST20 (Fig. 4G-H). These do not appear to be a result of high temperature alteration or metamorphism but the partial resorption of the highly altered, early plagioclase may represent small patches of felsic partial melt extracted from the surrounding gabbros.

\subsection{Whole-rock geochemistry}

Gabbros have a narrow $\mathrm{SiO}_{2}$ range between 46 and 54\% (Table 1, Fig. 5). The most mafic layered cumulate sample (SBST19) has relatively high $\mathrm{MgO}$ (11\%), $\mathrm{Mg \#} \mathrm{(67),} \mathrm{CaO}$ (13.8\%), Cr (960 ppm) and $\mathrm{Ni}$ (193 ppm), which is reflected by the presence of normative olivine and clinopyroxene as well as abundant hornblende. This sample is geochemically distinct from the other more evolved gabbro and leucogabbro samples, which have lower $\mathrm{MgO}$ values (4.38-8.36\%), $\mathrm{Mg \#} \mathrm{(31-43),} \mathrm{CaO}$ (6.7-9.9\%), $\mathrm{Cr}$ (50-390 ppm) and $\mathrm{Ni}$ (20-136 ppm). Most of the gabbros are characterized by low $\mathrm{K}_{2} \mathrm{O}$ (0.18-0.84\%) and moderate $\mathrm{TiO}_{2}(0.35-1.25 \%), \mathrm{Fe}_{2} \mathrm{O}_{3}(6-12 \%), \mathrm{P}_{2} \mathrm{O}_{5}$ (0.05-0.15\% but generally $<0.08), \mathrm{Zr}(18-83 \mathrm{ppm}), \mathrm{Nb}(0.2-3 \mathrm{ppm}), \mathrm{Y}$ (6.5-25 ppm). Spongtang Massif gabbros initially follow a trend showing early iron enrichment (Fig. 5C) due to early olivine and pyroxene crystallization until about $52 \% \mathrm{SiO}_{2}(\mathrm{MgO} 12-5 \%$ ) followed by rapid drop in iron content as hornblende dominates the crystallization process along with accessory magnetite. Both $\mathrm{Sr}$ (resident in plagioclase) and $\mathrm{Al}_{2} \mathrm{O}_{3}$ display flat trends when plotted against $\mathrm{MgO}$ indicating that plagioclase was not being crystallized following its early crystallization with olivine and clinopyroxene. This matches petrographic descriptions, which show highly altered plagioclase enveloped in fresh hornblende in most samples (Fig. 4A-E). Fractionation trends involving iron enrichment are typical of ophiolites unrelated to subduction in which anhydrous crystallization of water undersaturated magmas is the norm (Dilek and Furnes, 2011; Miyashiro, 1975). However, the hornblende-rich gabbros from the Spongtang Massif suggest that early crystallization of unsaturated tholeiitic magma shifted to crystallization of water saturated, hydrous melts and is more consistent with the combination of calc-alkaline and tholeiitic magmas found in island arc environments (Miyashiro, 1973; Pearce and Robinson, 2010). 
Table 1

Whole rock geochemistry.

\begin{tabular}{|c|c|c|c|c|c|c|c|c|c|c|c|c|c|c|c|c|c|}
\hline Sample & SBST08 & SBST09 & SBST01 & SBST04 & SBST07 & SBST02 & SBST03 & SBST15 & SBST22 & SBST19 & SBST11 & SBST16 & SBST17 & SBST06 & SBST18 & SBST05 & SBST20 \\
\hline Rock type & Basalt & Basalt & Basalt & Basalt & Basalt & Basalt & Basalt & Basalt dyke & Basalt & Gabbro & Gabbro & Gabbro & Gabbro & Gabbro & Gabbro & Gabbro & Gabbro pegmatite \\
\hline Latitude (N) & 34.05675 & 34.05675 & 34.051733 & 34.051733 & 34.05675 & 34.051733 & 34.051733 & 34.05997 & 34.06513 & 34.05997 & 34.06214 & 34.05997 & 34.05997 & 34.05465 & 34.05997 & 34.05277 & 34.05997 \\
\hline Longitude (E) & 76.77718 & 76.77718 & 76.771300 & 76.771300 & 76.77718 & 76.771300 & 76.771300 & 76.77534 & 76.77939 & 76.77534 & 76.78197 & 76.77534 & 76.77534 & 76.77379 & 76.77534 & 76.77240 & 76.77534 \\
\hline \multicolumn{18}{|l|}{ Majors (\%) } \\
\hline $\mathrm{SiO}_{2}$ & 46.60 & 46.84 & 46.86 & 47.02 & 47.04 & 48.65 & 48.99 & 52.69 & 52.16 & 46.63 & 46.94 & 50.52 & 51.18 & 52.37 & 53.11 & 54.29 & 45.67 \\
\hline $\mathrm{TiO}_{2}$ & 1.59 & 1.96 & 1.94 & 1.49 & 1.33 & 1.69 & 1.71 & 0.97 & 0.74 & 0.35 & 1.25 & 0.73 & 1.01 & 0.60 & 0.80 & 0.87 & 0.15 \\
\hline $\mathrm{Al}_{2} \mathrm{O}_{3}$ & 15.13 & 14.18 & 15.96 & 15.03 & 15.58 & 15.77 & 17.35 & 14.69 & 19.37 & 15.88 & 16.02 & 14.66 & 14.53 & 15.09 & 14.97 & 15.76 & 27.86 \\
\hline $\mathrm{Fe}_{2} \mathrm{O}_{3}$ & 11.83 & 11.34 & 11.55 & 9.47 & 9.77 & 9.20 & 12.62 & 12.18 & 4.60 & 6.08 & 8.55 & 11.24 & 13.81 & 10.69 & 10.11 & 10.63 & 1.29 \\
\hline MnO & 0.18 & 0.19 & 0.24 & 0.14 & 0.15 & 0.14 & 0.15 & 0.18 & 0.13 & 0.12 & 0.14 & 0.18 & 0.19 & 0.18 & 0.15 & 0.16 & 0.02 \\
\hline $\mathrm{MgO}$ & 6.59 & 8.12 & 7.10 & 7.07 & 8.02 & 7.10 & 4.71 & 3.26 & 1.84 & 11.13 & 5.92 & 8.36 & 5.38 & 4.46 & 5.47 & 4.38 & 1.85 \\
\hline $\mathrm{CaO}$ & 11.00 & 8.56 & 8.45 & 10.68 & 10.27 & 10.16 & 4.19 & 9.05 & 3.89 & 13.83 & 9.89 & 7.27 & 6.47 & 10.03 & 8.15 & 6.74 & 13.87 \\
\hline $\mathrm{Na}_{2} \mathrm{O}$ & 3.34 & 3.97 & 3.38 & 3.48 & 3.50 & 3.79 & 5.99 & 5.52 & 6.32 & 1.96 & 4.93 & 3.52 & 4.88 & 3.91 & 5.49 & 5.24 & 4.84 \\
\hline $\mathrm{K}_{2} \mathrm{O}$ & 0.20 & 0.12 & 0.83 & 0.13 & 0.10 & 0.28 & 0.41 & 0.62 & 4.96 & 0.65 & 0.25 & 0.32 & 0.31 & 0.84 & 0.18 & 0.23 & 0.42 \\
\hline $\mathrm{P}_{2} \mathrm{O}_{5}$ & 0.17 & 0.25 & 0.26 & 0.15 & 0.17 & 0.18 & 0.19 & 0.09 & 0.25 & 0.05 & 0.15 & 0.06 & 0.07 & 0.07 & 0.08 & 0.08 & 0.01 \\
\hline $\mathrm{SO}_{3}$ & 0.22 & 1.23 & 0.00 & 0.02 & 0.04 & 0.02 & $<0.01$ & 0.01 & 0.04 & 0.01 & 0.01 & 0.00 & 0.01 & 0.00 & $<0.01$ & $<0.01$ & $<0.01$ \\
\hline LOI & 3.21 & 3.44 & 3.43 & 5.54 & 3.44 & 3.36 & 3.01 & 0.85 & 5.11 & 3.2 & 6.32 & 3.16 & 2.53 & 2.08 & 1.56 & 1.1 & 3.29 \\
\hline Total & 100.06 & 100.06 & 100.00 & 100.22 & 99.43 & 100.34 & 99.32 & 100.10 & 99.43 & 99.43 & 100.36 & 100.01 & 100.37 & 100.32 & 100.06 & 99.47 & 99.47 \\
\hline \multicolumn{18}{|c|}{ Trace elements (ppm) } \\
\hline $\mathrm{Ba}$ & 29.4 & 17.8 & 56.1 & 11 & 14.8 & 17 & 16.4 & 58 & 500 & 25.5 & 27 & 23.8 & 46.3 & 227 & 15.7 & 18.2 & 12.6 \\
\hline $\mathrm{Ce}$ & 103 & 19.9 & 17.9 & 10.6 & 10.3 & 12.1 & 11.7 & 6.9 & 499 & 6.2 & 13.9 & 5.7 & 143 & 4.3 & 5.7 & 6.5 & 1.2 \\
\hline $\mathrm{Cr}$ & 350 & 310 & 320 & 350 & 420 & 360 & 390 & 60 & 40 & 960 & 390 & 100 & 50 & 60 & 150 & 50 & 80 \\
\hline Cs & 0.24 & 0.29 & 0.6 & 0.09 & 0.15 & 0.17 & 0.29 & 0.08 & 15.05 & 0.24 & 0.13 & 0.13 & 0.29 & 0.56 & 0.06 & 0.06 & 0.11 \\
\hline Dy & 5.34 & 6.23 & 5.85 & 5.03 & 4.42 & 5.27 & 6.47 & 3.43 & 3.15 & 2.47 & 3.92 & 2.89 & 2.94 & 2.87 & 3.39 & 3.42 & 0.87 \\
\hline $\mathrm{Er}$ & 3.61 & 3.89 & 3.73 & 3.4 & 2.88 & 3.37 & 4.06 & 2.26 & 1.59 & 1.69 & 2.53 & 2.06 & 2.03 & 2.07 & 2.24 & 2.4 & 0.76 \\
\hline $\mathrm{Eu}$ & 1.26 & 1.57 & 1.6 & 1.27 & 1.22 & 1.26 & 1.47 & 0.85 & 1.74 & 0.55 & 0.99 & 0.64 & 0.76 & 0.55 & 0.73 & 0.76 & 0.2 \\
\hline $\mathrm{Ga}$ & 17.1 & 17 & 17.4 & 15.9 & 16.9 & 15.4 & 15.3 & 16.5 & 38.1 & 12.9 & 14.1 & 14.9 & 18 & 14 & 15.4 & 16.3 & 20.9 \\
\hline Gd & 4.76 & 5.62 & 5.22 & 4.53 & 4.08 & 4.62 & 5.7 & 2.95 & 4.13 & 2.05 & 3.63 & 2.52 & 2.28 & 2.26 & 2.66 & 2.9 & 0.59 \\
\hline $\mathrm{Hf}$ & 2.8 & 3.4 & 3.1 & 2.5 & 2.2 & 2.8 & 2.6 & 1.7 & 29.2 & 0.5 & 1.9 & 1.4 & 1.8 & 1.1 & 1.6 & 1.6 & 0.2 \\
\hline Ho & 1.19 & 1.35 & 1.29 & 1.13 & 0.99 & 1.16 & 1.4 & 0.82 & 0.59 & 0.56 & 0.9 & 0.68 & 0.66 & 0.64 & 0.77 & 0.77 & 0.22 \\
\hline $\mathrm{La}$ & 3.3 & 5.3 & 6.7 & 3.3 & 3.5 & 3.9 & 5.8 & 2.3 & 47.1 & 2.4 & 3.6 & 1.9 & 1.6 & 1.5 & 1.9 & 2.3 & 0.7 \\
\hline Lu & 0.49 & 0.56 & 0.56 & 0.48 & 0.4 & 0.5 & 0.55 & 0.38 & 0.25 & 0.24 & 0.35 & 0.3 & 0.33 & 0.33 & 0.36 & 0.37 & 0.11 \\
\hline $\mathrm{Nb}$ & 1.8 & 3.1 & 6.3 & 2.2 & 2.1 & 2.6 & 2.5 & 1.3 & 529 & 1.4 & 3 & 1 & 0.9 & 0.8 & 1 & 1.2 & 0.2 \\
\hline $\mathrm{Nd}$ & 8.9 & 13.1 & 13.1 & 9.5 & 8.3 & 10.1 & 11.4 & 5.5 & 30.4 & 3.9 & 7.6 & 4.6 & 3.8 & 3.9 & 5.1 & 5.5 & 0.7 \\
\hline $\operatorname{Pr}$ & 1.69 & 2.66 & 2.61 & 1.78 & 1.63 & 1.95 & 2.23 & 1 & 8.69 & 0.82 & 1.48 & 0.85 & 0.71 & 0.67 & 0.95 & 1.03 & 0.12 \\
\hline $\mathrm{Rb}$ & 5.6 & 1.3 & 18.3 & 2.4 & 1.6 & 5 & 6.1 & 6.2 & 125 & 8.2 & 4 & 3 & 7.6 & 8.3 & 1.9 & 3.5 & 11.3 \\
\hline $\mathrm{Sm}$ & 3.03 & 4.31 & 4.04 & 3.16 & 2.83 & 3.52 & 3.74 & 1.99 & 5.06 & 1.27 & 2.74 & 1.57 & 1.41 & 1.49 & 1.81 & 1.94 & 0.24 \\
\hline Sn & 182 & 8 & 2 & 2 & 2 & 2 & 2 & 1 & 786 & 2 & 10 & 1 & 266 & 2 & 2 & 2 & 1 \\
\hline $\mathrm{Sr}$ & 116.5 & 163.5 & 221 & 92.7 & 149.5 & 79.9 & 67.3 & 278 & 321 & 166 & 137.5 & 87.5 & 135.5 & 65.9 & 159 & 100.5 & 89.9 \\
\hline Ta & 0.1 & 0.2 & 0.5 & 0.2 & 0.2 & 0.3 & 0.2 & 0.1 & 43.4 & 0.1 & 0.2 & 0.1 & 0.1 & 0.1 & 0.1 & 0.1 & $<0.1$ \\
\hline $\mathrm{Tb}$ & 0.86 & 1.03 & 0.96 & 0.85 & 0.73 & 0.89 & 1.04 & 0.53 & 0.64 & 0.37 & 0.66 & 0.46 & 0.44 & 0.42 & 0.52 & 0.54 & 0.12 \\
\hline Th & 0.15 & 0.23 & 0.57 & 0.16 & 0.17 & 0.17 & 0.2 & 0.15 & 36.2 & 0.2 & 0.2 & 0.12 & 0.19 & 0.15 & 0.13 & 0.14 & $<0.05$ \\
\hline $\mathrm{Tm}$ & 0.52 & 0.59 & 0.58 & 0.52 & 0.44 & 0.52 & 0.6 & 0.37 & 0.25 & 0.26 & 0.38 & 0.3 & 0.33 & 0.33 & 0.34 & 0.34 & 0.13 \\
\hline U & 0.09 & 0.1 & 0.19 & 0.07 & 0.08 & 0.08 & 0.17 & 0.09 & 4.57 & 0.05 & 0.11 & 0.08 & 0.11 & 0.1 & 0.09 & 0.08 & $<0.05$ \\
\hline V & 293 & 298 & 329 & 278 & 277 & 303 & 290 & 375 & 71 & 201 & 222 & 310 & 530 & 245 & 312 & 296 & 141 \\
\hline W & $<1$ & 1 & $<1$ & $<1$ & 1 & 1 & $<1$ & 2 & $<1$ & 3 & $<1$ & 2 & 3 & 1 & 2 & $<1$ & 1 \\
\hline Y & 33.7 & 39.1 & 36.2 & 31.7 & 28.7 & 33.1 & 42.4 & 21.7 & 13.7 & 15.9 & 24.8 & 19.3 & 18.7 & 19.2 & 21.8 & 22.4 & 6.5 \\
\hline $\mathrm{Yb}$ & 3.24 & 3.61 & 3.47 & 3.04 & 2.75 & 3.18 & 3.71 & 2.36 & 1.61 & 1.42 & 2.3 & 1.92 & 2.02 & 1.93 & 2.27 & 2.31 & 0.71 \\
\hline $\mathrm{Zr}$ & 116 & 152 & 122 & 96 & 89 & 107 & 101 & 57 & 2170 & 18 & 83 & 48 & 65 & 36 & 55 & 57 & 4 \\
\hline
\end{tabular}


Table 2

U-Pb SHRIMP data.

\begin{tabular}{|c|c|c|c|c|c|c|c|c|c|c|c|c|c|c|}
\hline \multirow{2}{*}{$\begin{array}{l}\text { Labels } \\
\text { SBST-12 }\end{array}$} & \multirow[t]{2}{*}{ Site } & \multirow[t]{2}{*}{$\mathrm{U} / \mathrm{ppm}$} & \multirow[t]{2}{*}{ Th/ppm } & \multirow[t]{2}{*}{ Th/U } & \multirow[t]{2}{*}{$\%$ f206 } & \multicolumn{3}{|c|}{${ }^{238} \mathrm{U} /{ }^{206} \mathrm{~Pb}$ (meas) } & \multicolumn{3}{|c|}{${ }^{207} \mathrm{~Pb} /{ }^{206} \mathrm{~Pb}$ (meas) } & \multicolumn{3}{|c|}{ Age ${ }^{206} \mathrm{~Pb} /{ }^{238} \mathrm{U}$ (corr) } \\
\hline & & & & & & & & & & & & & & \\
\hline 1.1 & $\mathrm{~m}, \mathrm{osc}, \mathrm{eq}$ & 375 & 424 & 1.13 & 0.85 & 46.33 & \pm & 1.27 & 0.0556 & \pm & 0.0017 & 136.5 & \pm & 2.7 \\
\hline 2.1 & $\mathrm{~m}, \mathrm{osc} / \mathrm{h}, \mathrm{fr}$ & 188 & 175 & 0.93 & 2.29 & 47.23 & \pm & 1.10 & 0.0619 & \pm & 0.0022 & 132.0 & \pm & 4.0 \\
\hline 3.1 & $\mathrm{~m}, \mathrm{osc} / \mathrm{h}, \mathrm{eq}$ & 348 & 337 & 0.97 & 1.10 & 46.38 & \pm & 0.80 & 0.0556 & \pm & 0.0015 & 136.0 & \pm & 2.0 \\
\hline 4.1 & $\mathrm{~m}, \mathrm{osc}$, eq & 583 & 339 & 0.58 & 0.43 & 46.36 & \pm & 0.59 & 0.0520 & \pm & 0.0009 & 137.0 & \pm & 1.4 \\
\hline 5.1 & m, osc, eq, fr & 313 & 328 & 1.05 & 0.95 & 45.74 & \pm & 1.03 & 0.0564 & \pm & 0.0015 & 138.1 & \pm & 2.5 \\
\hline 6.1 & $\mathrm{e}, \mathrm{osc}, \mathrm{p}, \mathrm{fr}$ & 592 & 376 & 0.64 & 0.43 & 47.80 & \pm & 0.85 & 0.0523 & \pm & 0.0012 & 132.9 & \pm & 1.9 \\
\hline 7.1 & $\mathrm{~m}, \mathrm{osc} / \mathrm{h}, \mathrm{eq}, \mathrm{fr}$ & 409 & 727 & 1.78 & 0.30 & 47.18 & \pm & 1.45 & 0.0511 & \pm & 0.0013 & 134.8 & \pm & 3.4 \\
\hline 8.1 & $\mathrm{e}, \mathrm{osc}, \mathrm{p}, \mathrm{fr}$ & 342 & 529 & 1.55 & 0.73 & 46.56 & \pm & 1.76 & 0.0544 & \pm & 0.0017 & 136.0 & \pm & 3.7 \\
\hline 9.1 & e, osc, p, fr & 217 & 201 & 0.93 & 1.78 & 46.00 & \pm & 0.94 & 0.0626 & \pm & 0.0021 & 136.2 & \pm & 3.1 \\
\hline 10.1 & $\mathrm{e}, \mathrm{osc} / \mathrm{h}, \mathrm{p}$ & 566 & 874 & 1.54 & 0.42 & 47.06 & \pm & 0.73 & 0.0523 & \pm & 0.0011 & 135.0 & \pm & 1.6 \\
\hline 11.1 & $\mathrm{e}, \mathrm{h}, \mathrm{p}$ & 496 & 438 & 0.88 & 0.33 & 47.82 & \pm & 0.94 & 0.0510 & \pm & 0.0013 & 133.0 & \pm & 2.2 \\
\hline 12.1 & $\mathrm{e}, \mathrm{h}, \mathrm{p}$ & 361 & 356 & 0.99 & 0.41 & 45.46 & \pm & 0.78 & 0.0521 & \pm & 0.0011 & 139.7 & \pm & 1.8 \\
\hline \multicolumn{15}{|l|}{ SBST-05 } \\
\hline 1.1 & tiny, h/osc & 846 & 2026 & 2.39 & 1.26 & 47.36 & \pm & 0.96 & 0.0585 & \pm & 0.0035 & 133.0 & \pm & 3.5 \\
\hline 2.1 & tiny, h/osc & 1105 & 2807 & 2.54 & 0.83 & 47.94 & \pm & 1.11 & 0.0550 & \pm & 0.0016 & 132.0 & \pm & 2.2 \\
\hline 3.1 & tiny, h/osc & 1383 & 9802 & 7.09 & 0.21 & 44.81 & \pm & 2.48 & 0.0530 & \pm & 0.0014 & 142.0 & \pm & 5.5 \\
\hline 4.1 & tiny, h/osc & 1488 & 6853 & 4.60 & 1.11 & 57.46 & \pm & 2.22 & 0.0568 & \pm & 0.0014 & 110.0 & \pm & 3.0 \\
\hline
\end{tabular}

Isotopic ratios uncorrected for common $\mathrm{Pb}$.

Spot: $\mathrm{x} . \mathrm{y}=$ grain followed by analysis number.

Site: $\mathrm{m}=$ middle, $\mathrm{e}=$ end, $\mathrm{p}=$ prismatic grain, $\mathrm{eq}=$ equant grain, osc $=$ oscillatory zoned, $\mathrm{h}=$ homogeneous, $\mathrm{fr}=\mathrm{fragment}$.

\% 206: percentage of ${ }^{206} \mathrm{~Pb}$ that is non-radiogenic (common).

Ages corrected for common Pb by the '207' method (modelled as concordant).

All analytical errors are 1 sigma.

Chondrite normalized REE patterns for the Spongtang gabbro are generally flat with a slight depletion in the light rare earth elements (LREE) compared to the flat, and slightly more elevated patterns of the basalts (Fig. 6A). An exception is a pronounced positive Ce anomaly for a gabbro (SBST17) and one basalt sample (SBST08). While negative Ce anomalies reflect the subduction of pelagic sediments and seawater alteration (Hole et al., 1984), positive Ce anomalies are more likely to be associated with the highly oxidizing, fluid-rich nature of the gabbroic melts that formed in a supra-subduction zone setting and had fractionated past silica-saturation and started crystallizing zircon, which unlike most minerals strongly partitions $\mathrm{Ce}^{4+}$ but not $\mathrm{Ce}^{3+}$ (Ballard et al., 2002). Eu anomalies do not occur in any samples indicating that plagioclase was not fractionated during magmatic crystallization (Pallister and Knight, 1981). Middle to heavy REE patterns are flat for gabbros and basalts reflecting early olivine, plagioclase and pyroxene crystallization while the slight, relative depletion in LREE is consistent with a depleted mantle source and/or late stage crystallization of hornblende, which does not retain LREE (Kocak et al., 2005). High-field strength (HFS) element ( $\mathrm{Hf}, \mathrm{Zr}, \mathrm{Ti}, \mathrm{Nb}$ and $\mathrm{Ta}$ ) concentrations are low to moderate, reflecting a slight depletion associated with subduction related magmas (Pearce, 1975). Both gabbro and basalt samples display negative Nb-Ta anomalies (Fig. 6F) due to the compatible and immobile nature of these elements in oxidizing supra-subduction zone conditions (Pearce, 2008). However, there is a distinct enrichment of mobile and incompatible large ion lithophile (LIL) elements (Fig. 6F) typical of subduction-related magmas (Pearce and Robinson, 2010).

Tectonic discrimination plots (Pearce, 1982; Pearce, 2008; Pearce and Cann, 1973; Shervais, 1982) show basalts of the Spongtang Massif generally plot in the N-MORB field. However, basaltic andesites of the Spong Arc plot in the island arc field (Fig. 6A-D). Most Photang Valley gabbro samples plot in the island arc field with a few exceptions plotting in the N-MORB field. The Th/Yb-Nb/Yb plot of Pearce (2008) clearly demonstrates the N-MORB affinities of the Spongtang Massif basalts and the departure to the island arc field for the younger Spong Arc

Table 3

Hf data.

\begin{tabular}{|c|c|c|c|c|c|c|c|c|c|c|c|c|c|c|}
\hline \multirow{2}{*}{$\begin{array}{l}\text { Analysis } \\
1.1\end{array}$} & \multicolumn{3}{|c|}{${ }^{176} \mathrm{Lu} /{ }^{177} \mathrm{Hf}^{\mathrm{a}}$} & \multicolumn{3}{|c|}{ Measured ${ }^{176} \mathrm{Hf} /{ }^{177} \mathrm{Hf}$} & \multicolumn{3}{|c|}{$\begin{array}{l}\varepsilon_{\mathrm{Hf}}(0) \text { in run errors } \\
\text { only }^{\mathrm{b}}\end{array}$} & \multirow{2}{*}{$\begin{array}{l}\text { U-Pb age (Ma) } \\
136.5\end{array}$} & \multirow{2}{*}{$\begin{array}{l}\text { Initial }{ }^{176} \mathrm{Hf} /{ }^{177} \mathrm{Hf} \\
0.28312\end{array}$} & \multicolumn{2}{|l|}{$\varepsilon_{\mathrm{Hf}}(\mathrm{t})$} & \multirow{2}{*}{$\begin{array}{l}\text { 2SE } \\
0.8\end{array}$} \\
\hline & 0.00328 & \pm & 0.00003 & 0.28313 & \pm & 0.00001 & 12.11 & \pm & 0.40 & & & 14.9 & \pm & \\
\hline 2.1 & 0.00639 & \pm & 0.00001 & 0.28319 & \pm & 0.00002 & 14.37 & \pm & 0.60 & 132 & 0.28318 & 16.8 & \pm & 1.20 \\
\hline 3.1 & 0.00595 & \pm & 0.00011 & 0.28317 & \pm & 0.00001 & 13.61 & \pm & 0.49 & 136 & 0.28315 & 16.1 & \pm & 1.0 \\
\hline 4.1 & 0.00544 & \pm & 0.00009 & 0.28315 & \pm & 0.00001 & 13.09 & \pm & 0.51 & 137 & 0.28314 & 15.6 & \pm & 1.0 \\
\hline 5.1 & 0.00571 & \pm & 0.00001 & 0.28320 & \pm & 0.00001 & 14.91 & \pm & 0.44 & 138.1 & 0.28319 & 17.5 & \pm & 0.9 \\
\hline 6.1 & 0.00585 & \pm & 0.00006 & 0.28313 & \pm & 0.00001 & 12.14 & \pm & 0.48 & 132.9 & 0.28311 & 14.6 & \pm & 1.0 \\
\hline 8.1 & 0.00402 & \pm & 0.00001 & 0.28320 & \pm & 0.00001 & 14.66 & \pm & 0.45 & 134.8 & 0.28319 & 17.3 & \pm & 1.0 \\
\hline 9.1 & 0.00285 & \pm & 0.00006 & 0.28315 & \pm & 0.00001 & 12.97 & \pm & 0.39 & 136 & 0.28314 & 15.7 & \pm & 0.8 \\
\hline 10.1 & 0.00738 & \pm & 0.00001 & 0.28320 & \pm & 0.00002 & 14.70 & \pm & 0.59 & 136.2 & 0.28318 & 17.1 & \pm & 1.2 \\
\hline 11.1 & 0.00668 & \pm & 0.00002 & 0.28315 & \pm & 0.00001 & 12.98 & \pm & 0.52 & 135 & 0.28314 & 15.4 & \pm & 1.0 \\
\hline 12.1 & 0.00369 & \pm & 0.00006 & 0.28315 & \pm & 0.00001 & 12.91 & \pm & 0.42 & 133 & 0.28314 & 15.5 & \pm & 0.8 \\
\hline A & 0.01245 & \pm & 0.00039 & 0.28313 & \pm & 0.00002 & 12.36 & \pm & 0.70 & 137 & 0.28310 & 14.3 & \pm & 1.4 \\
\hline B & 0.00675 & \pm & 0.00007 & 0.28325 & $\overline{ \pm}$ & 0.00003 & 16.45 & \pm & 0.93 & 137 & 0.28323 & 18.9 & $\overline{ \pm}$ & 1.8 \\
\hline$C$ & 0.00344 & \pm & 0.00007 & 0.28319 & \pm & 0.00002 & 14.37 & \pm & 0.69 & 137 & 0.28318 & 17.1 & \pm & 1.4 \\
\hline $\mathrm{D}$ & 0.00517 & \pm & 0.00007 & 0.28319 & \pm & 0.00001 & 14.36 & \pm & 0.48 & 137 & 0.28318 & 16.9 & \pm & 1.0 \\
\hline E & 0.00551 & \pm & 0.00004 & 0.28314 & \pm & 0.00001 & 12.73 & \pm & 0.43 & 137 & 0.28313 & 15.3 & \pm & 0.9 \\
\hline $\mathrm{F}$ & 0.00355 & \pm & 0.00006 & 0.28315 & \pm & 0.00001 & 12.81 & \pm & 0.39 & 137 & 0.28314 & 15.5 & \pm & 0.8 \\
\hline G & 0.00648 & \pm & 0.00004 & 0.28318 & \pm & 0.00001 & 14.07 & \pm & 0.47 & 137 & 0.28317 & 16.5 & \pm & 1.0 \\
\hline
\end{tabular}

a Initial ratios are calculated at either the SHRIMP U-Pb age (Table 2) or at the average age of $137 \mathrm{Ma}$. Uncertainty in initial ratios are given as $2 \mathrm{SE}$.

b \&Hf values are calculated using CHUR parameters given in Bouvier et al., 2008. 

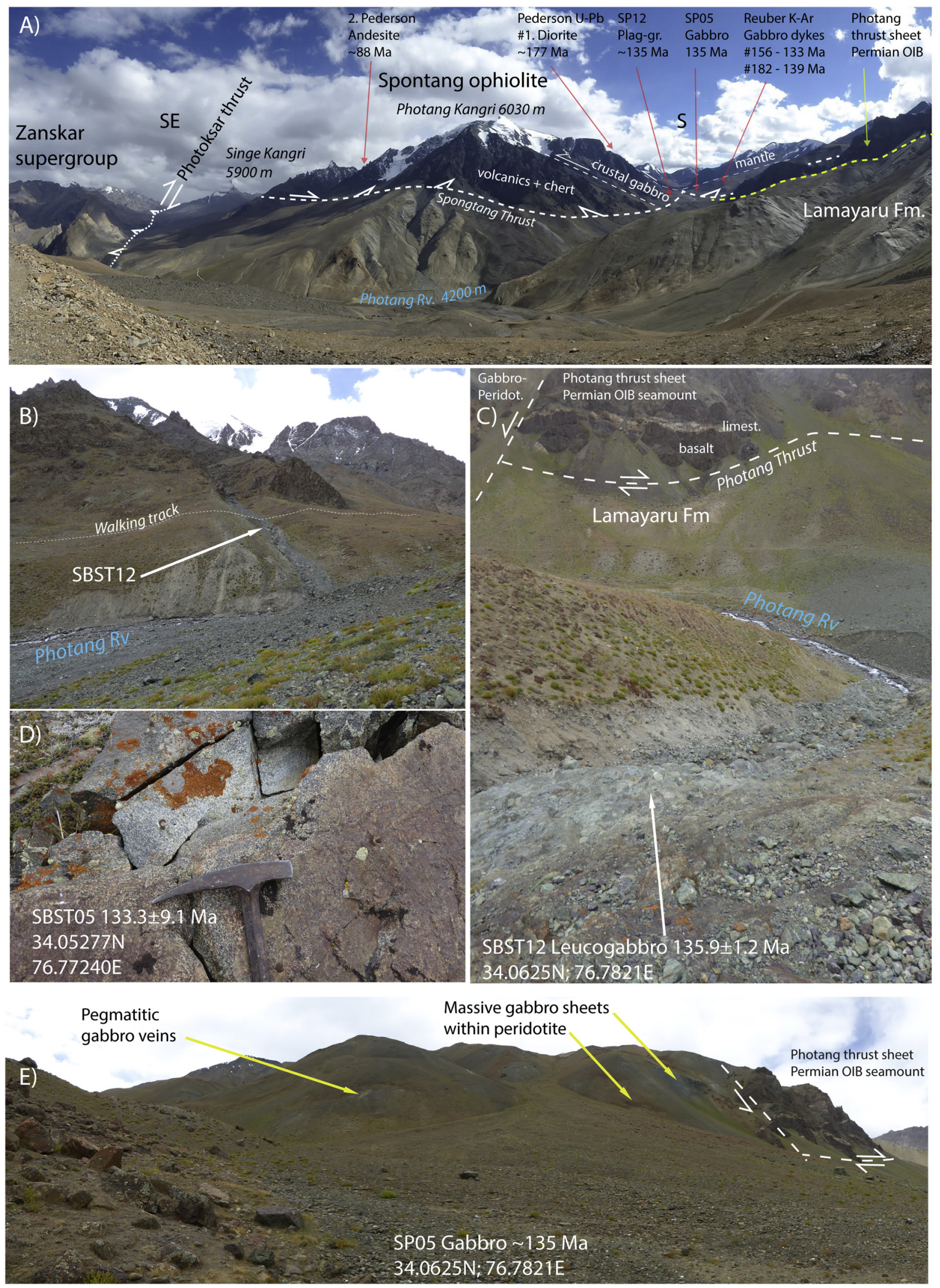

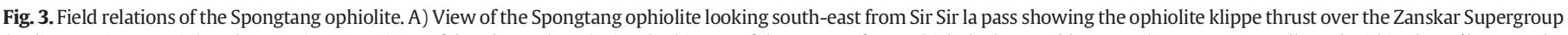

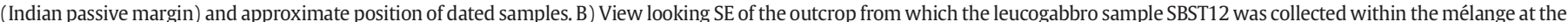

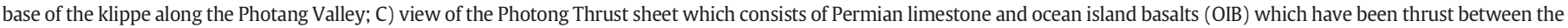

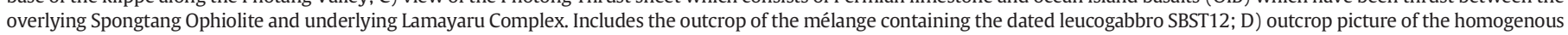
gabbro SBST05; E) view of the pegmatitic gabbro dykes and veins intruding massive gabbro sheets and peridotite in the upper Photang Valley. 

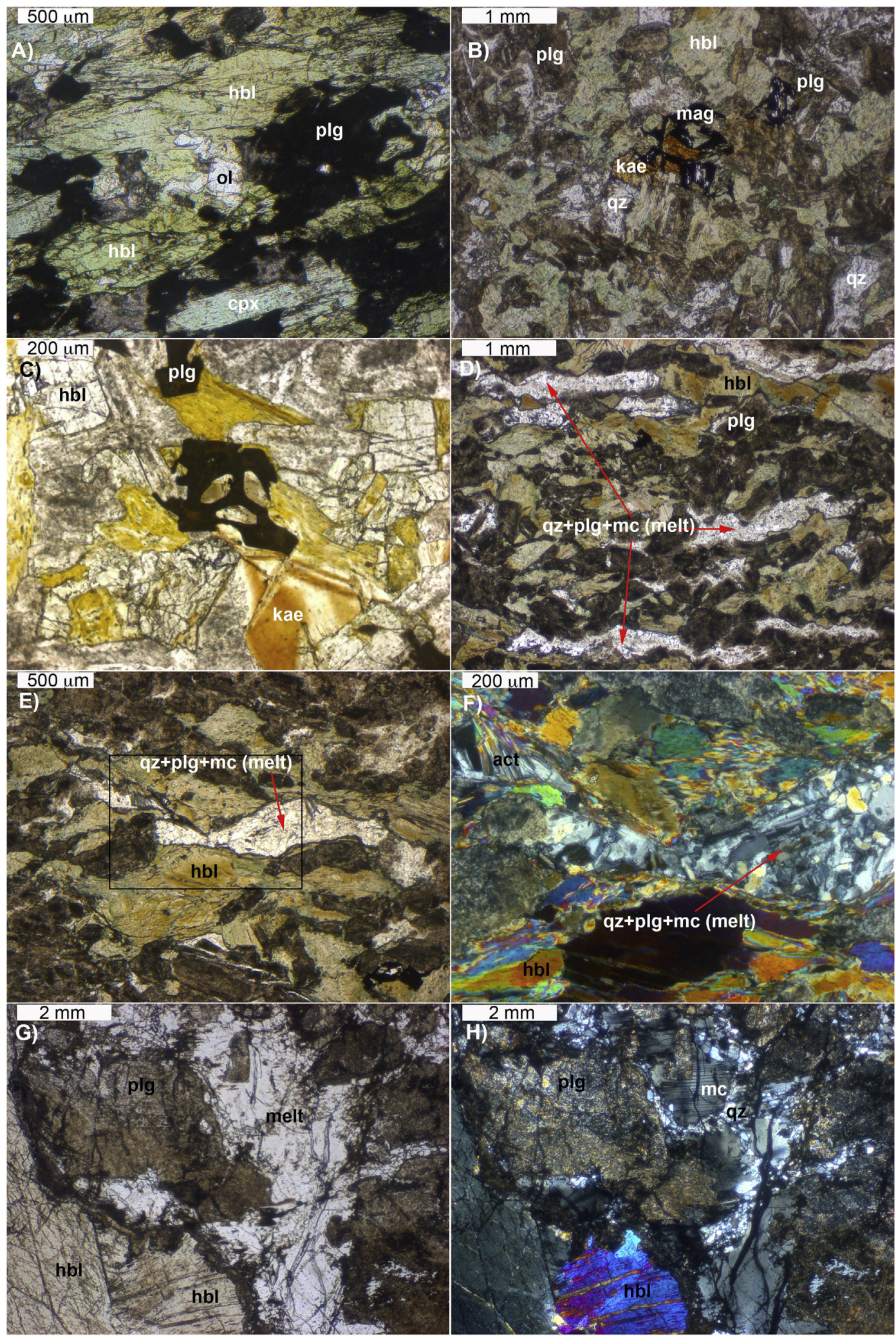

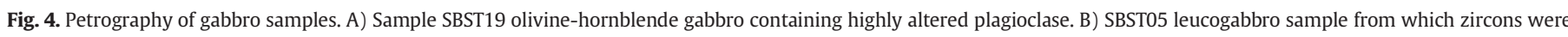

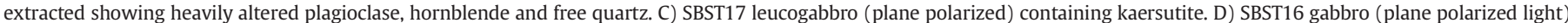

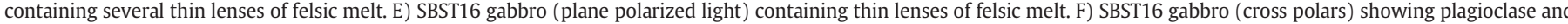

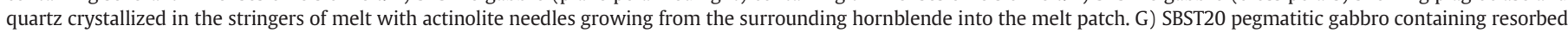
plagioclase, hornblende, kaersutite H) SBST20 pegmatitic gabbro (cross polars) showing the cross-hatch twinning of microcline that has crystallized with quartz in the melt veins.

samples (Fig. 6C). The gabbros collected in this study show a gradual but clear displacement from the basalts falling on the MORB-OIB array towards the island arc field (Fig. 6C). Increasing $\mathrm{Th} / \mathrm{Yb}$ ratio reflects the gradual increase of subducted sediments shed off the evolving island arc and recycled back into the gabbro melt while the slight decrease in $\mathrm{Nb} / \mathrm{Yb}$ ratio reflects the conservative, immobile and compatible nature 
A)

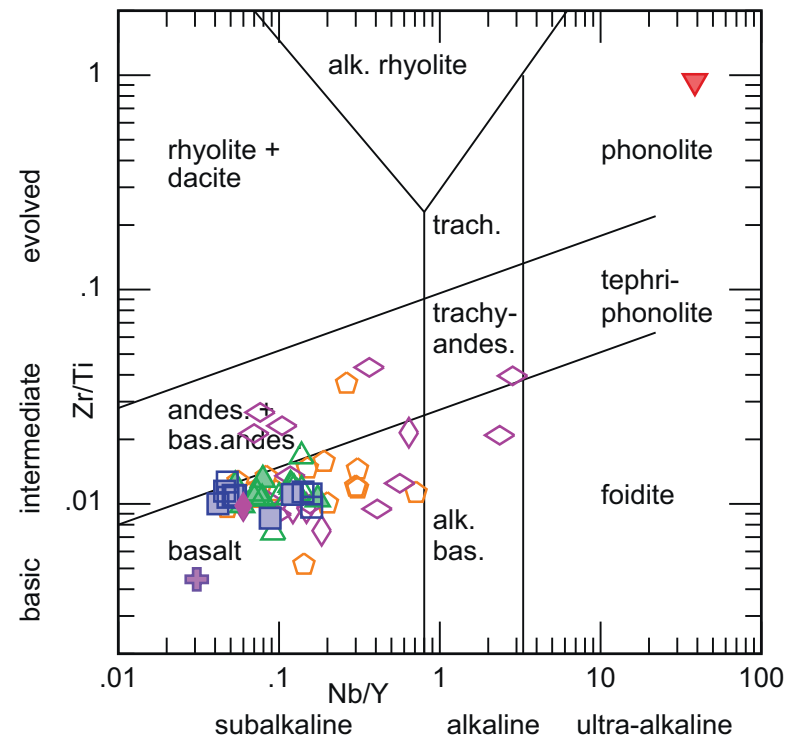

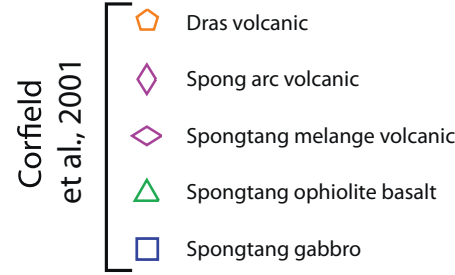

$\begin{array}{lll}\triangle & \text { Spongtang ophiolite basalt } \\ \frac{\sim}{2} & \checkmark & \text { Spongtang dyke } \\ \frac{n}{\models} & \square & \text { Spongtang pegmatitic gabbro } \\ \square & \text { Spongtang gabbro } \\ \nabla & \text { Photang thrust sheet OlB seamount }\end{array}$
B)

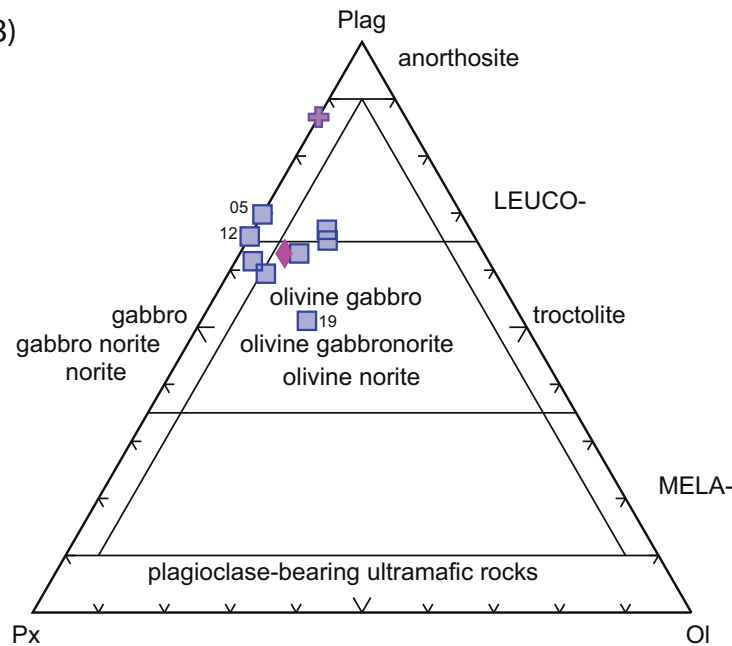

C)

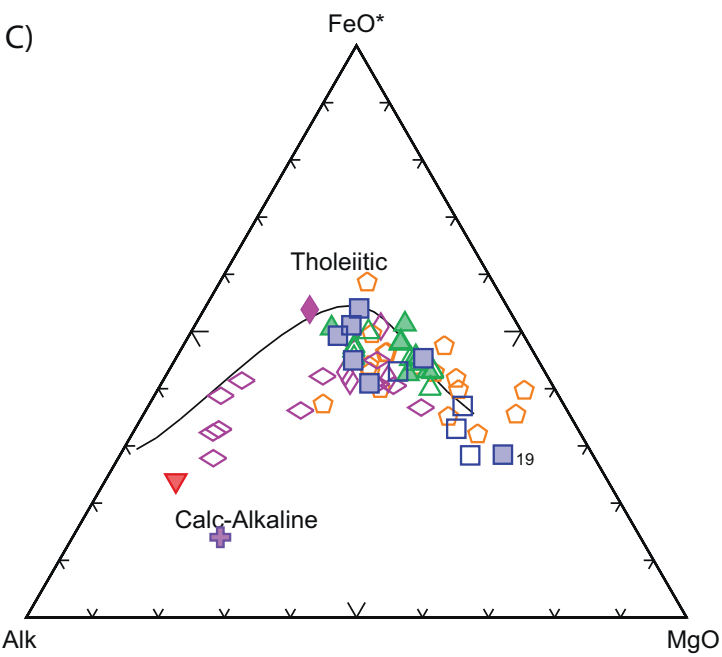

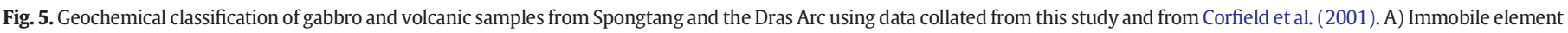
rock classification diagram (Winchester and Floyd, 1977). B) IUGS gabbro classification. C) The tholeiitic-calc-alkaline classification (Irvine and Baragar, 1971).

of $\mathrm{Nb}$ in highly oxidized environments such as subduction zones (Pearce, 2008). The discrimination plots of $\mathrm{V} / \mathrm{Ti} * 1000$ (Shervais, 1982), Ti/Zr (Pearce and Cann, 1973) and Th-Ti-Zr/117 (Wood, 1980) all show a clear discrimination of the N-MORB basalts from the island arc gabbros (Fig. 6A-D). This supports field observations in which gabbro dykes and sills intruded the volcanic rocks and mantle peridotites, and suggests there was a second stage of magmatism associated with island arc development that was superimposed on pre-existing N-MORB type crust or MORB-like fore-arc crust associated with the earliest phase of subduction, for example, Izu-Bonin-Marianas arc basement (Ishizuka et al., 2018).

\subsection{SHRIMP U-Pb zircon results}

Approximately $1-2 \mathrm{~kg}$ of both gabbro samples SBST05 and -12 gave zircon yields of $\sim 50(50-100 \mu \mathrm{m})$ and $\sim 100(100-250 \mu \mathrm{m})$ grains respectively. Sample SBST05 zircons are cloudy yellow, prismatic grains and fragments, in which oscillatory zoning is widely disrupted by recrystallization (Fig. 7). SBST12 zircons are translucent to pale yellow, euhedral, oscillatory zoned and of equant to stubby prismatic habit (Fig. 7). The small size of zircon grains in sample
SBST05 considerably reduced the choice of sites for analysis. Zircons from sample SBST12 are markedly larger, which afforded a greater choice of sites for analysis.

For gabbro sample SBST12, 12 analyses were undertaken on 12 grains. Sites from CL images indicating recrystallization were avoided (Fig. 7, Table 2). The chosen sites have U content of 188-583 ppm, with elevated $\mathrm{Th} / \mathrm{U}$ of $0.55-1.55$. These elevated $\mathrm{Th} / \mathrm{U}$ values are typical of zircons crystallized from intermediate to gabbroic magmas (e.g., Paces and Miller, 1993). Uncorrected for minor amounts of common $\mathrm{Pb}$, all sites yield close to concordant $\mathrm{U}-\mathrm{Pb}$ ages, with all having indistinguishable ${ }^{238} \mathrm{U} /{ }^{206} \mathrm{~Pb}$, but with small dispersion in ${ }^{207} \mathrm{~Pb} /{ }^{206} \mathrm{~Pb}$ (Fig. 8). Those with the highest ${ }^{207} \mathrm{~Pb} /{ }^{206} \mathrm{~Pb}$ show marginally higher amounts of common $\mathrm{Pb}$ (Table 2). After correction for common $\mathrm{Pb}$ by the '207' method (Compston et al., 1984), all analyses yield a weighted mean ${ }^{206} \mathrm{~Pb} /{ }^{238} \mathrm{U}$ age of $135.9 \pm 1.2 \mathrm{Ma}$ (95\% confidence, MSWD = 1.05).

For gabbro sample SBST05, only four analyses were completed due to the small and partially metamict state of the grains (Table 2). Other analyses were attempted but aborted, due to the small size of grains and much higher levels of common $\mathrm{Pb}$ being present (judged from high ${ }^{204} \mathrm{~Pb}$ count rates in the first peak-hop cycle). The SBST05 zircons 
A)

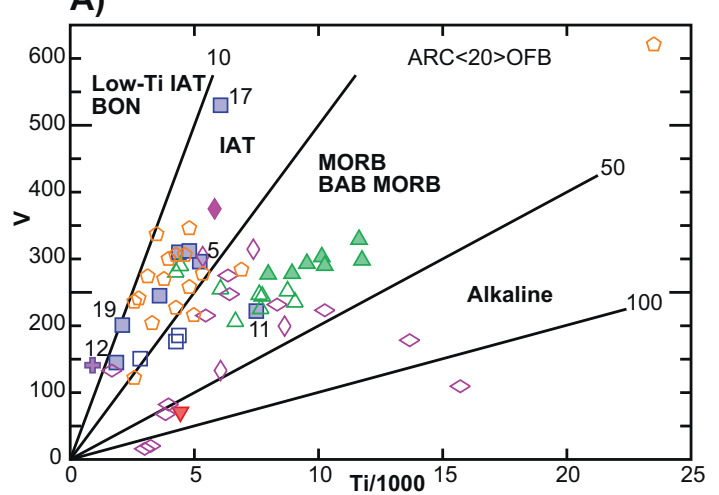

10 C)
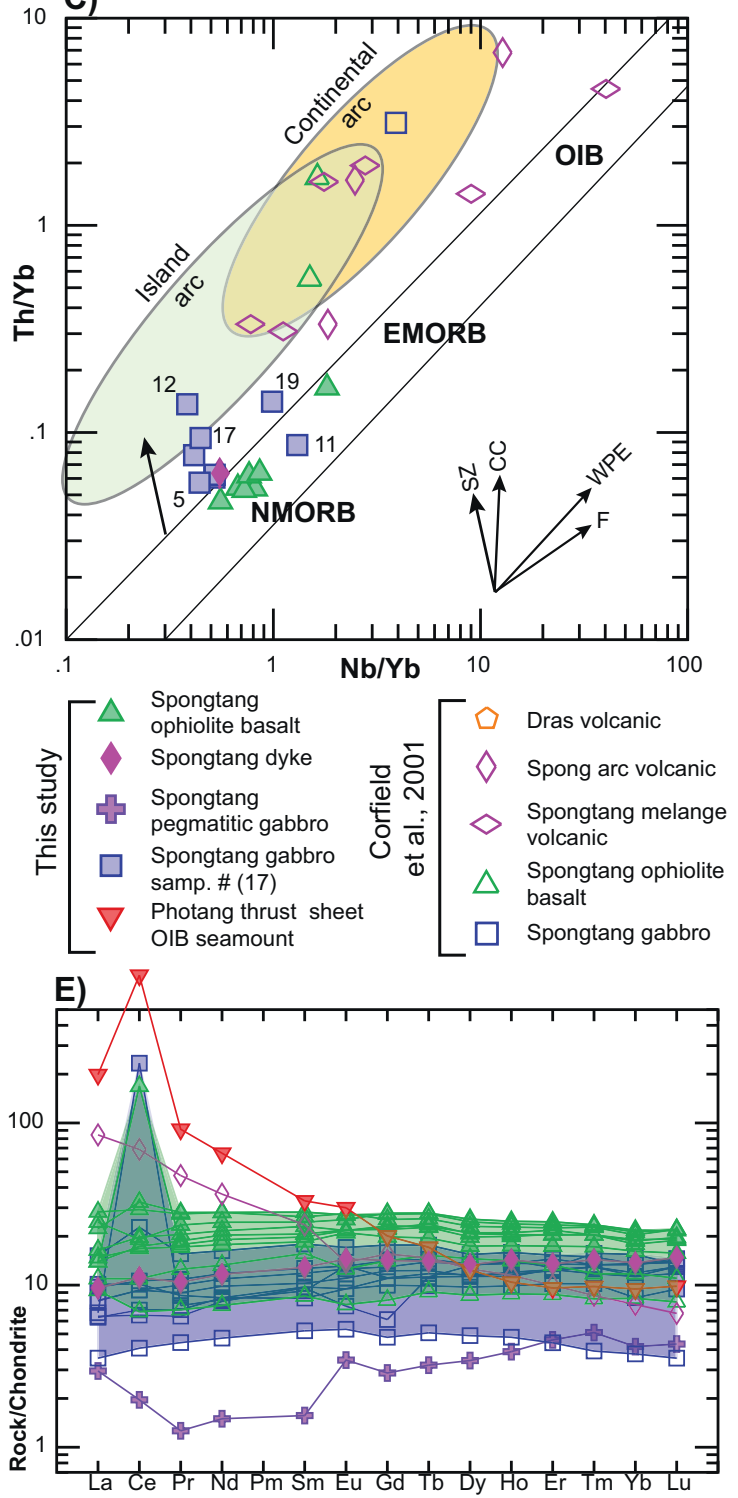

B)

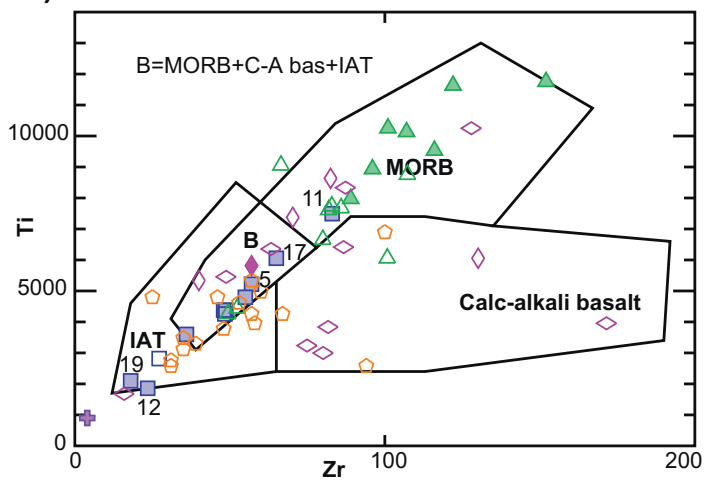

D)

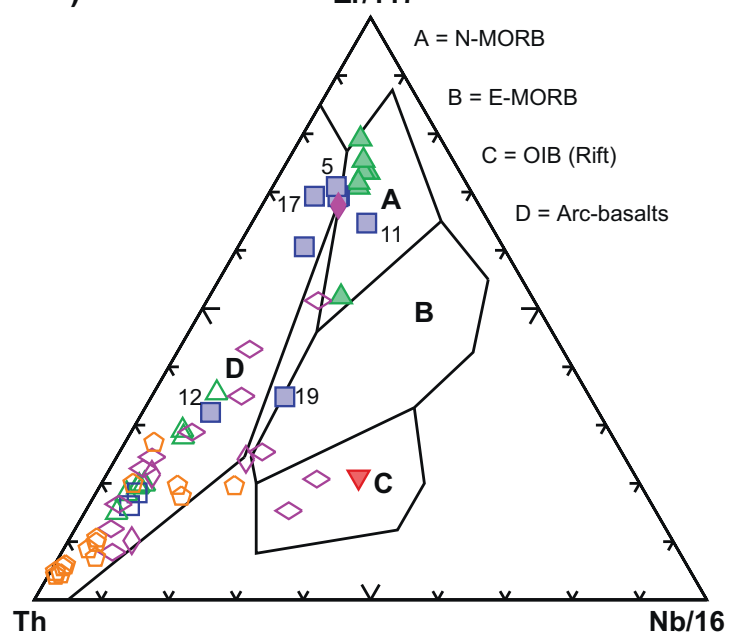

F)

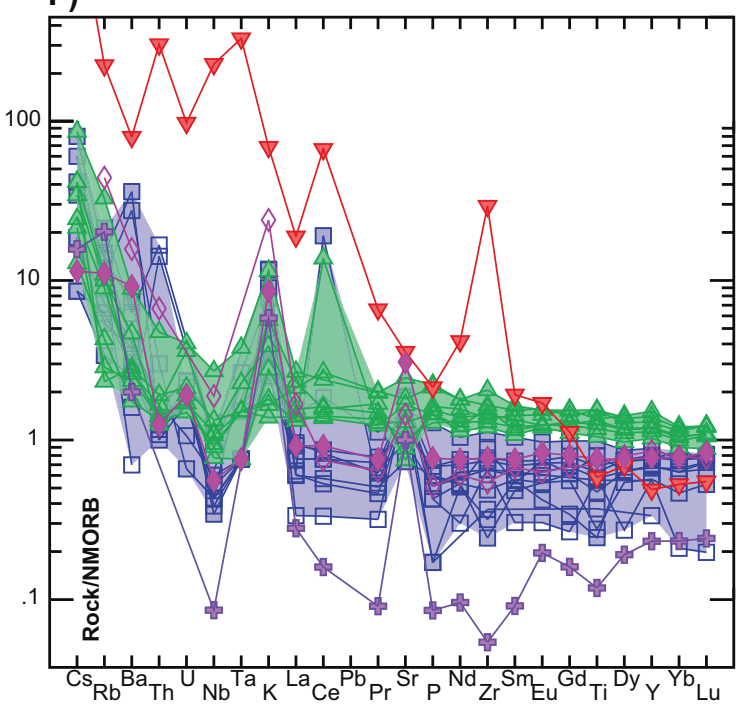

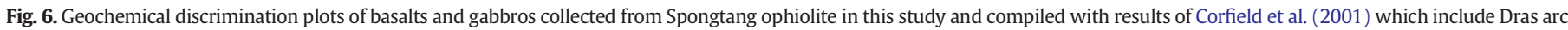

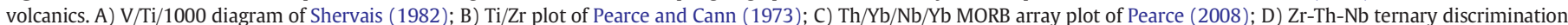
diagram of Wood (1980); E) chondrite normalized and F) NMORB normalized rare-earth plot of Sun and McDonough (1989).

have considerably higher U content (846-1488 ppm), with very high $\mathrm{Th} / \mathrm{U}$ (2.39-7.09). These grains are interpreted to be magmatic in origin, formed from U-enriched melt at the last stage of crystallization of the intermediate-gabbroic magma. The completed analyses have higher common $\mathrm{Pb}$ content than those in sample SBST12, and uncorrected for common $\mathrm{Pb}$ form a slightly discordant population with real dispersion in ${ }^{238} \mathrm{U} /{ }^{206} \mathrm{~Pb}$ and ${ }^{207} \mathrm{~Pb} /{ }^{206} \mathrm{~Pb}$ (Fig. 8). Analysis 4.1 with high $\mathrm{U}$ and high common $\mathrm{Pb}$ gives an apparently younger ${ }^{206} \mathrm{~Pb} /{ }^{238} \mathrm{U}$ age than the 


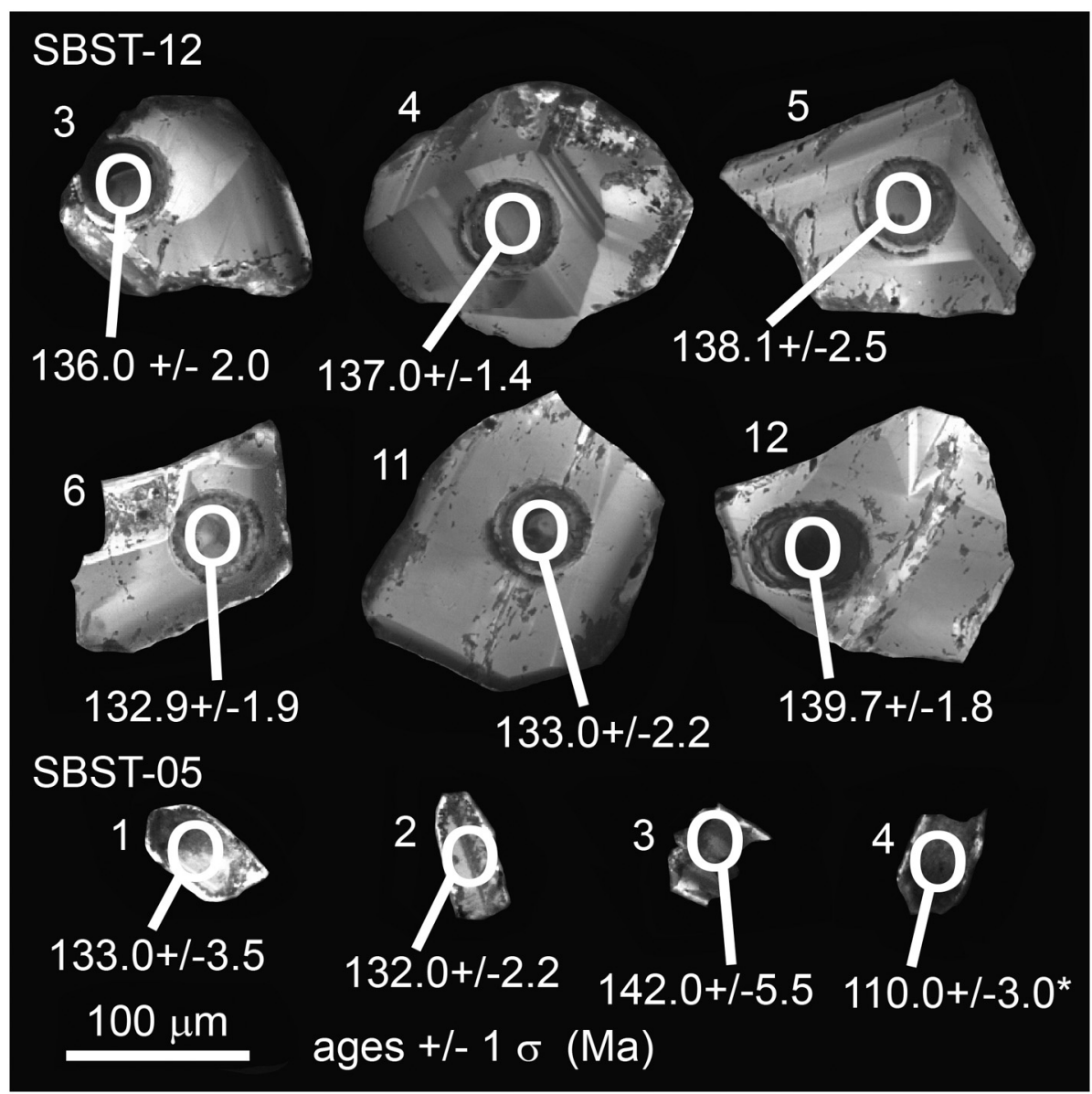

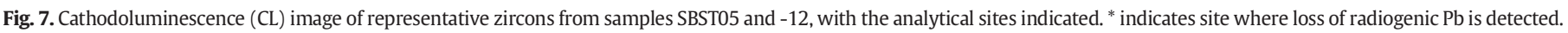

other sites and might have lost some radiogenic $\mathrm{Pb}$ in a recent event. After correction for common $\mathrm{Pb}$ by the '207' method, analyses 1.1 , 2.1 and 3.1 give a weighted mean ${ }^{206} \mathrm{~Pb} /{ }^{238} \mathrm{U}$ age $133.3 \pm 9.1 \mathrm{Ma}$ ( $95 \%$ confidence, MSWD = 4.1). Within its larger error (due to the lesser number of analyses), this is indistinguishable from the age of the SBST12 zircons. These ages indicate magmatic crystallization of samples SBST05 and SBST12 at 136-133 Ma (Early Cretaceous; Hauterivian-Valanginian).

\subsection{LA-ICP-MS Lu-Hf results}

Due to the small size of the SBST05 zircons, LA-MC-ICP-MS analyses were not attempted, and only SBST12 grains were analyzed (Table 3 ). Eleven analyses were undertaken over SHRIMP U-Pb analysis sites, together with an additional seven analyses on sites free of evidence of recrystallization from CL images, but without prior U-Pb analysis. Present day $\varepsilon_{\mathrm{Hf}}$ ranges from +12.1 to +14.9 (all $\varepsilon_{\mathrm{Hf}}$ values are calculated using a present day CHUR composition of ${ }^{176} \mathrm{Hf} /{ }^{177} \mathrm{Hf}=0.282785$ and ${ }^{176} \mathrm{Lu} /{ }^{177} \mathrm{Hf}=0.0336$; Bouvier et al., 2008) with minor spread beyond analytical error, whereas $\varepsilon_{\mathrm{Hf}}$ at $136 \mathrm{Ma}$ - the time of igneous crystallization of the zircons, ranges from +14.3 to +18.9 with a weighted mean value of $16.0 \pm 0.5$ ( $95 \%$ confidence). These values are equal to estimates for depleted MORB source mantle at that time. Hence, there is no evidence of any crustal contamination in the mantle source from subducted older crustal materials or through mixing in the arc roots with older crust, as is seen in most arc suites (as summarized by Jones et al., 2015).

\section{Discussion}

\subsection{Geochronology and $\mathrm{Hf}$ isotope signatures}

Heitz (1986) first reported a whole-rock K-Ar age of $140 \pm 15 \mathrm{Ma}$ for Spongtang Massif basalts. Reuber et al. (1989) obtained several K-Ar ages from basic dykes collected from the Spongtang Massif. They yielded two clusters of amphibole ages - the first between 140 and $125 \mathrm{Ma}$ which they interpreted as the age of dyke intrusions and the second at about 170 Ma which they interpreted as the minimum age of formation of the ophiolite. It should be noted that most of the Spongtang Massif rocks have been metamorphosed to sub-greenschist facies and consequently $\mathrm{K}-\mathrm{Ar}$ dates are unlikely to be reliable given the mobility of K and Ar during metamorphism. Mahéo et al. (2004) undertook ${ }^{40} \mathrm{Ar}^{-39} \mathrm{Ar}$ dating on amphiboles of the Spongtang Massif, which yielded ages between 130 and $110 \mathrm{Ma}$. Pedersen et al. (2001) reported a TIMS single zircon age of a diorite from Spongtang Massif at $177 \pm 1 \mathrm{Ma}$, which they interpreted to be the age of the ophiolite basement before development of the Spong Arc. Whether this Jurassic age represents the original MORB ocean crust or an early phase of supra-subduction zone ophiolite-arc development is difficult to determine. This Jurassic age is similar to the Zedong terrane in Tibet (McDermid et al., 2002) but older than most other ophiolite fragments along the IYTS, such as Xigaze at 126 Ma (Aitchison et al., 2003; Malpas et al., 2003). Pedersen et al. (2001) also reported that zircons from andesitic rocks of the overlying Spong Arc volcaniclastic sequence yielded an age of $88 \pm 5 \mathrm{Ma}$, which they interpreted to constrain the minimum age of subduction initiation beneath the Spongtang Massif. Our samples are 


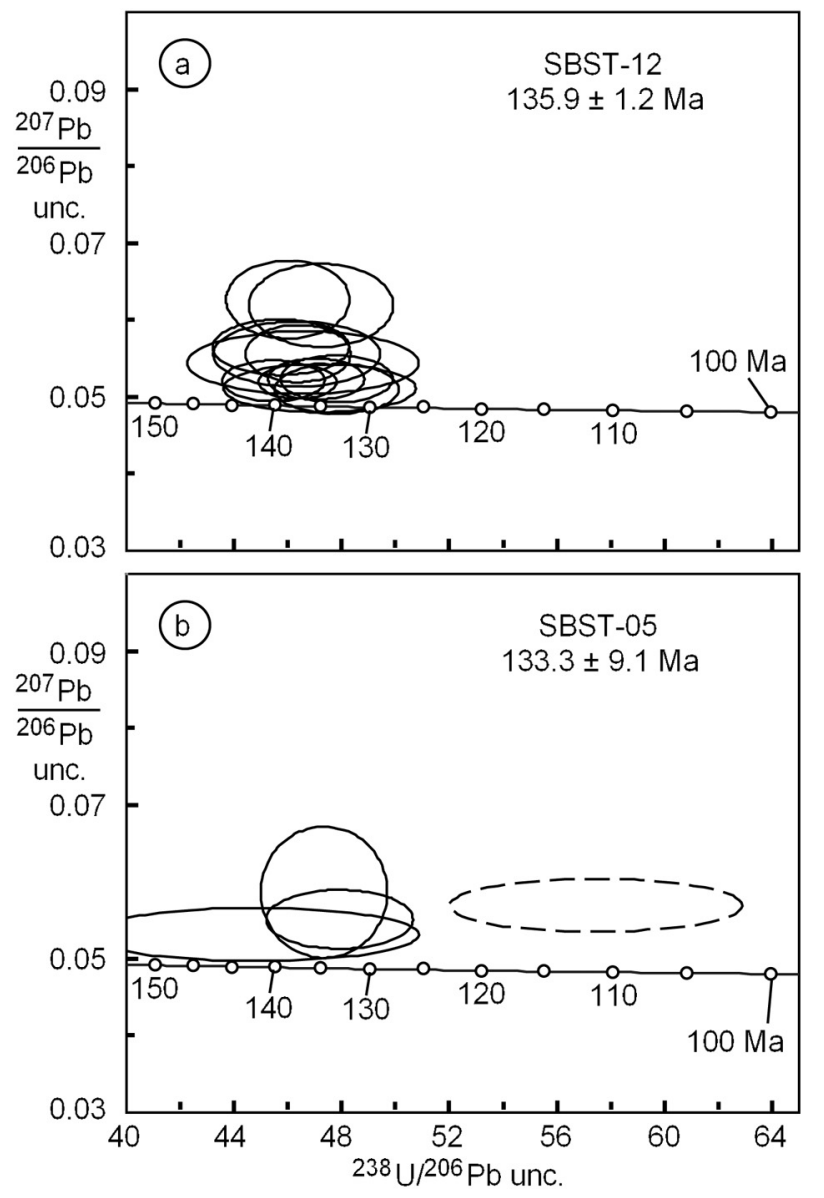

Fig. 8. TW. ${ }^{238} \mathrm{U} /{ }^{206} \mathrm{~Pb}-{ }^{207} \mathrm{~Pb} /{ }^{206} \mathrm{~Pb}$ plot, uncorrected for common $\mathrm{Pb}$. Analytical errors are depicted at the 2 sigma level.

of gabbros with arc-like whole rock geochemistry that yielded magmatic zircons of $136 \mathrm{Ma}$ and $133 \mathrm{Ma}$. The Cretaceous ( 136 Ma) gabbros, which intrude both the mantle and volcanic ophiolite sections have a distinct supra-subduction geochemical signature (Fig. 6) and possibly signify intra-oceanic subduction initiation and fore-arc spreading in $\sim 177 \mathrm{Ma}$ oceanic crust, which led to the development of the Spong island-arc during the Cretaceous. The Hf isotopic data obtained from SBST12 with initial $\varepsilon_{\mathrm{Hf}}$ of +14.3 to +18.9 show that this sample formed in a setting free of detectable contamination by older continental crust (Fig. 9). This shows there was neither distally or locally derived continental trench sediment nor extended older continental crust in the roots of the arc.

The Cretaceous U-Pb zircon age of $\sim 136$ Ma we report here differs from previous Jurassic U-Pb age of $\sim 177 \mathrm{Ma}$ of Pedersen et al. (2001) but is similar to the Ar-Ar ages provided by Mahéo et al. (2004). We suggest this older Jurassic ages relates to fragments of Neotethyan, MORB-like, oceanic crust of the Spongtang ophiolite, on top of which the Spong Arc developed following Early Cretaceous subduction initiation.

Lower Cretaceous (mid-Valanginian-mid-Aptian range) radiolarian faunal assemblages reported by Baxter et al. (2010) provide robust biostratigraphic ages for the ophiolite-arc complex. This Lower Cretaceous assignment correlates with the younger population of K-Ar dates obtained by Reuber (1989) and our U-Pb zircon crystallization age. The similarity between ages for the early Spong Arc igneous rocks and cherts suggests that the cherts are a part of the lower section of the Spong Arc stratigraphy rather than being derived from older Jurassic ophiolite basement or older accreted fragments of the Neotethyan ocean crust scraped off the descending slab beneath the Spong Arc.

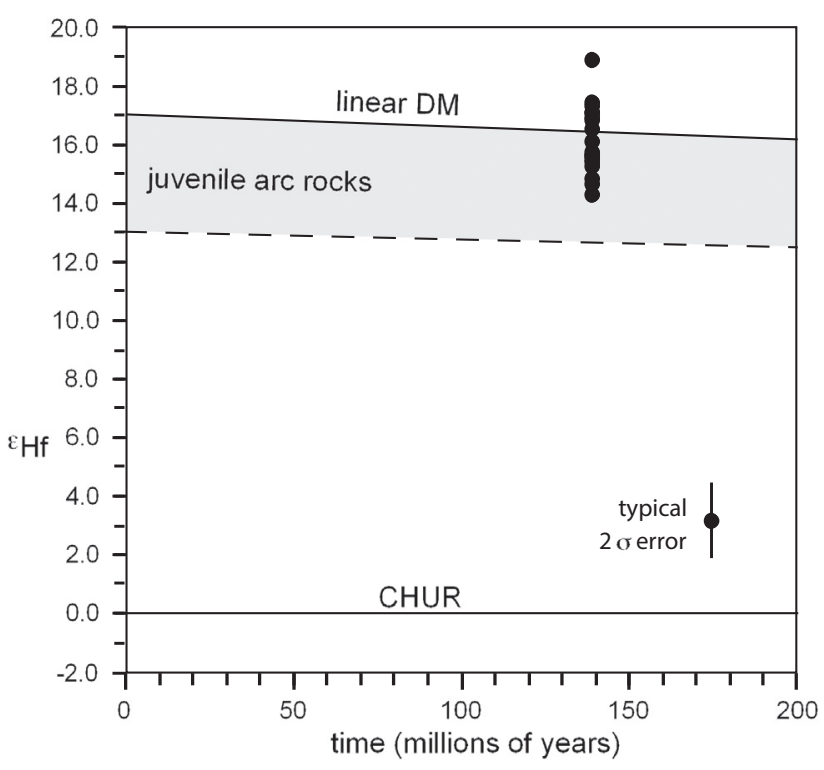

Fig. 9. Hf. $\varepsilon_{\mathrm{Hf}}$-time plot for the SBST12 zircon data. 'Linear DM' (depleted mantle) is a chord between a modern $\varepsilon_{\mathrm{Hf}}=+17$ and $\varepsilon_{\mathrm{Hf}}=0$ at $4560 \mathrm{Ma}$. CHUR is the Chondritic Uniform Reservoir reference. The field 'juvenile arc rocks' is bounded by linear DM and the evolution line of Dhuime et al. (2011) for island arcs - a chord from modern $\varepsilon_{\mathrm{Hf}}=$ +13 and $\varepsilon_{\mathrm{Hf}}=0$ at $4560 \mathrm{Ma}$. A typical $\pm 2 \sigma$ error bar for the data is shown.

Gabbro from the Nidar ophiolite to the east has been dated at 130-110 Ma by the amphibole ${ }^{40} \mathrm{Ar}^{39} \mathrm{Ar}$ method (Mahéo et al., 2004) and $140 \pm 32$ Ma using a nine point mineral-whole rock Sm-Nd isochron with an initial ${ }^{143} \mathrm{Nd} /{ }^{144} \mathrm{Nd}$ of $0.513835 \pm 0.000053\left(E_{\mathrm{Nd}} t=\right.$ +7.4) (Ahmad et al., 2008). Cherts within the overlying volcanosedimentary unit yield Lower Cretaceous (132-127 Ma) radiolarians (Kojima et al., 2001) consistent with Spongtang cherts. This ophiolite structurally overlies the Tso Morari metamorphic complex to the south, which contains subducted portions of the Indian continent including 55 Ma coesite-bearing ultra-high pressure eclogites (de Sigoyer et al., 2000; Donaldson et al., 2013; Leech et al., 2007; St-Onge et al., 2013). de Sigoyer et al. (2004) interpret these eclogites to represent subducted portions of Indian crust beneath Eurasia and therefore the age of continent-continent collision (Leech et al., 2005). However, like Spongtang, the Nidar ophiolite is intra-oceanic in origin (Ahmad et al., 2008; Zyabrev et al., 2008). It is thrust over eclogite-bearing Indian crustal rocks at Tso Morari, indicating it was the first element to collide with India and this event may predate the final continental collision. It is becoming increasingly evident that ophiolites along the Indus-Yarlung-Tsangpo Suture represent an intra-oceanic suprasubduction zone ophiolite/island arc complex that was obducted onto the Indian margin as part of a north-dipping subduction zone that was distinct and separate from the Ladakh-Kohistan Arc that was also active but nearer the southern Eurasian margin (Aitchison et al., 2007a). A second, continental "Andean-type" convergent margin on the southern margin of Eurasia is supported by detrital zircon studies which indicate the Trans-Himalayan Arc was active until at least $41 \mathrm{Ma}$ in the Ladakh Batholith (Henderson et al., 2011) and $37 \mathrm{Ma}$ in the Gandese Batholith (Aitchison et al., 2011), well after collision of the ophiolite-arc complexes with India at $\sim 55 \mathrm{Ma}$. The existence of two north-dipping subduction zones and two separate collisions is supported by the geological evidence. Nowhere along the entire Indus-Yarlung-Tsangpo suture do any of the Trans-Himalayan Arc igneous rocks intrude into the ophiolite-arc complexes as one would expect if they were the basement to a single subduction zone. The contact between the Ladakh Arc and ophiolitic complexes is always faulted and this relationship is the same in Tibet. Indeed, the double subduction zone model is testable and falsifiable by the presence of an intrusive relationship between 
the Ladakh Arc and ophiolite complexes. This relationship would disprove the double subduction-collision model but it hasn't been recorded anywhere along the entire suture.

\subsection{Intra-oceanic geodynamic setting of the Spong Arc}

The transfer of juvenile, intra-oceanic ophiolitic and island-arc crust onto continental margins is an important mechanism for continental growth and the ages of ophiolites and the timing of emplacement onto continental margins are critical to accurate tectonic reconstructions of collision zones. This process is recorded in modern arccontinent collision systems, for example, Oman (Searle and Cox, 1999), New Caledonia (Aitchison et al., 1995), Taiwan (Huang et al., 2000) and Papua New Guinea (Holm et al., 2015) and is the key mechanism for the on-land preservation of dense, oceanic crust that would otherwise be recycled at subduction zones back into the mantle. Arccontinent collisions are responsible for widespread deformation events within ancient accretionary orogens such as eastern Australia (Aitchison and Buckman, 2012) and Central Asia (Buckman and Aitchison, 2004). Notably, these can be very short-lived events as documented by Dewey (2005). Lawsonite-bearing blueschists within the Shergol melange indicate cold subduction took place in an intraoceanic setting beneath the Dras Arc (Groppo et al., 2016) at about $100 \mathrm{Ma}$ (Honegger et al., 1989) and continued until the collision and partial subduction of the northern Indian continental margin to form the Tso Morari eclogites at $\sim 55 \mathrm{Ma}$ (de Sigoyer et al., 2000). Further west in Pakistan, Kakar et al. (2012) extracted zircons from the Muslim Bagh ophiolite which yielded U-Pb crystallization ages of $\sim 80$ Ma while geological relations constrain the emplacement age to between 68 and $48 \mathrm{Ma}$. They correlate the Muslim Bagh ophiolite with the Spong Arc as an intra-oceanic ophiolite-arc complex that first collided with India before the final India-Eurasia continental collision.

The isotopically juvenile nature of the 136 Ma Spong Arc gabbros suggests that they developed far from the influence of any continental derived material and therefore, are unlikely to represent the fore-arc portion of the Trans-Himalayan Arc which grew at the southern Eurasian margin and shows clear isotopic evidence of continental influence within the Gangdese and Ladakh batholiths (Najman et al., 2017). This fits with existing paleomagnetic results indicating that the Ladakh Arc and southern margin of the Lhasa terrane were situated between 15 and $25^{\circ} \mathrm{N}$ throughout the Cretaceous, whereas the Indus-YarlungTsangpo Suture ophiolites (Dazhuqu terrane) formed at equatorial settings some 1000-2500 km further south (Abrajevitch et al., 2005). Thus, the two subduction systems were unrelated.

\subsection{Spong and Dras island arcs}

The flat-lying Spongtang Massif is situated only $19 \mathrm{~km}$ south of the Dras-Nindam unit (Fig. 10), being separated by a pop-up structure consisting of the Zanskar Supergroup that was thrust up and through the Dras-Nindam unit after final continental collision. The close proximity, similar ages and compositions of these rocks suggest that the Spong Arc may be equivalent to the Dras Arc (Fig. 10) and this is consistent with the early cross-section interpretations of Fuchs (1982) who previously classified the Spong Arc volcaniclastic rocks as "Dras Arc". The intra-oceanic island arc affinities of both units hints at their being part of the same subduction system rather than belonging to a separate systems as was suggested by Corfield et al. (2001).

The Spongtang Massif is unlikely to be part of the fore-arc region of the Kohistan-Ladakh-Gangdese Arc on the southern Eurasian margin (Fuchs, 1982; Steck, 2003) as it is more evolved and has a stronger continental influence as evident by the lower initial $\varepsilon_{\mathrm{Hf}}$ values (Bouilhol et al., 2011, 2013; Najman et al., 2017). Many tectonic models for the Indus Suture are heavily influenced by early interpretations that correlate the Kohistan, Ladakh and Dras arc systems as part of the same entity that evolved on the southern margin of Eurasia (Coward et al., 1987;
Honegger et al., 1982; Khan et al., 1993; Searle et al., 1999). Granites of the Kohistan and Ladakh batholiths are of the same composition and age range as the Gandese Batholith in Tibet and therefore, are most likely to be part of the same Trans-Himalayan continental margin magmatic belt (Honegger et al., 1982; White et al., 2011). However, inconsistencies arise when forcing correlations with the Dras Arc and interpretations that it formed either the fore-arc basement of the Kohistan-Ladakh Arc or was an island arc that collided with Eurasia before evolving into the Kohistan-Ladakh-Arc (Fuchs, 1982; Honegger et al., 1982; Najman et al., 2017; Robertson and Degnan, 1994). This interpretation is based on the observation that the Dras volcanics are intruded by the $103 \mathrm{Ma}$ Kargil intrusive rocks (gabbro-norites to granodiorites), which are interpreted by Honegger et al. (1982) as equivalents of the Kohistan-Ladakh Arc and therefore, the Dras Arc had to have collided with Eurasia during the Late Cretaceous prior to intrusion of the Kargil granodiorites and final collision with India.

Corfield et al. (2001) suggested that the Spong and Dras island arcs developed above two separate island-arc subduction systems unrelated to the Ladakh Arc on the southern margin of Eurasia. They suggest that these island-arc terranes only came together during final continental collision. However, zircon studies by Singh et al. (2007), Ravikant et al. (2009) and White et al. (2011) found no evidence for a 100 Ma magmatic phase within the Ladakh Arc that might match the Kargil intrusive rocks. Instead, extensive granite magmatism occurred within the Ladakh Arc between 66 and 46 Ma with minor 85-75 Ma inheritance. This is reflected in detrital zircon studies of Indus Group sedimentary rocks, which show a distinct magmatic peak at 40-60 Ma and a smaller peak at 80-90 Ma but very few older zircons (Henderson et al., 2011). We suggest that correlation of the $103 \mathrm{Ma}$ Kargil intrusive rocks with 66-46 Ma granites of the Ladakh Batholith is not justified and that the Kargil intrusive rocks may represent the plutonic core of the intraoceanic Dras island arc, which is unrelated to the Ladakh Arc that developed off the southern margin of Eurasia. Without the inferred correlation between Kargil intrusive rocks and Ladakh Batholith, there is no reason to support the interpretation of the Dras Arc first colliding with Eurasia and no reason to separate the Dras and Spong arcs as different island-arcs. Instead, we suggest that the Spong and Dras arcs are part of the same island arc complex that first collided with India during the early Eocene.

\subsection{Timing of ophiolite-arc collision with India and closure of the Neotethys}

The timing of collision of the Spongtang Massif with India is highly disputed as outlined in discussions between Garzanti and Searle (Garzanti et al., 2005). Corfield et al. (2001) argue for a Late Cretaceous collision between Spongtang Massif and India while Reuber et al. (1992) and Garzanti et al. (2005) argue for a collision later in the Eocene. The presence of Lower Eocene units (Chulung la Formation and Kong Slate) of the Zanskar Supergroup (India) beneath the Spongtang Massif thrust sheet was used as evidence that the ophiolite was not emplaced onto the Indian margin until post-Early Eocene (Colchen et al., 1987; Fuchs, 1982; Reuber, 1986; Reuber et al., 1992; Reuber et al., 2015). Several tectonic reconstructions place northern India at about $10^{\circ} \mathrm{N}$ (Aitchison et al., 2007a; Dewey et al., 1989; Molnar and Tapponnier, 1975) and at least $1000 \mathrm{~km}$ south of Eurasia at $45 \mathrm{Ma}$ when the Ladakh Arc on the southern margin of Eurasia was at $23^{\circ} \mathrm{N}$ (Klootwijk et al., 1979). However, Corfield et al. (2001) contend that ophiolite obduction occurred much earlier in the Late Cretaceous and that the thrusting of the ophiolite over restricted Eocene marine basins that developed prior to and adjacent to the obducted ophiolite is related to later continent-continent collision. Corfield et al. (2001) interpret the closure of the Neotethys as a three-stage collision process starting with the obduction of the intra-oceanic Spongtang Massif onto India at about the same time that the intra-oceanic Dras-Kohistan Arc collided with the Trans-Himalayan Batholith to the north. This was followed by closure of the remaining Neotethys along southern Asia and final 


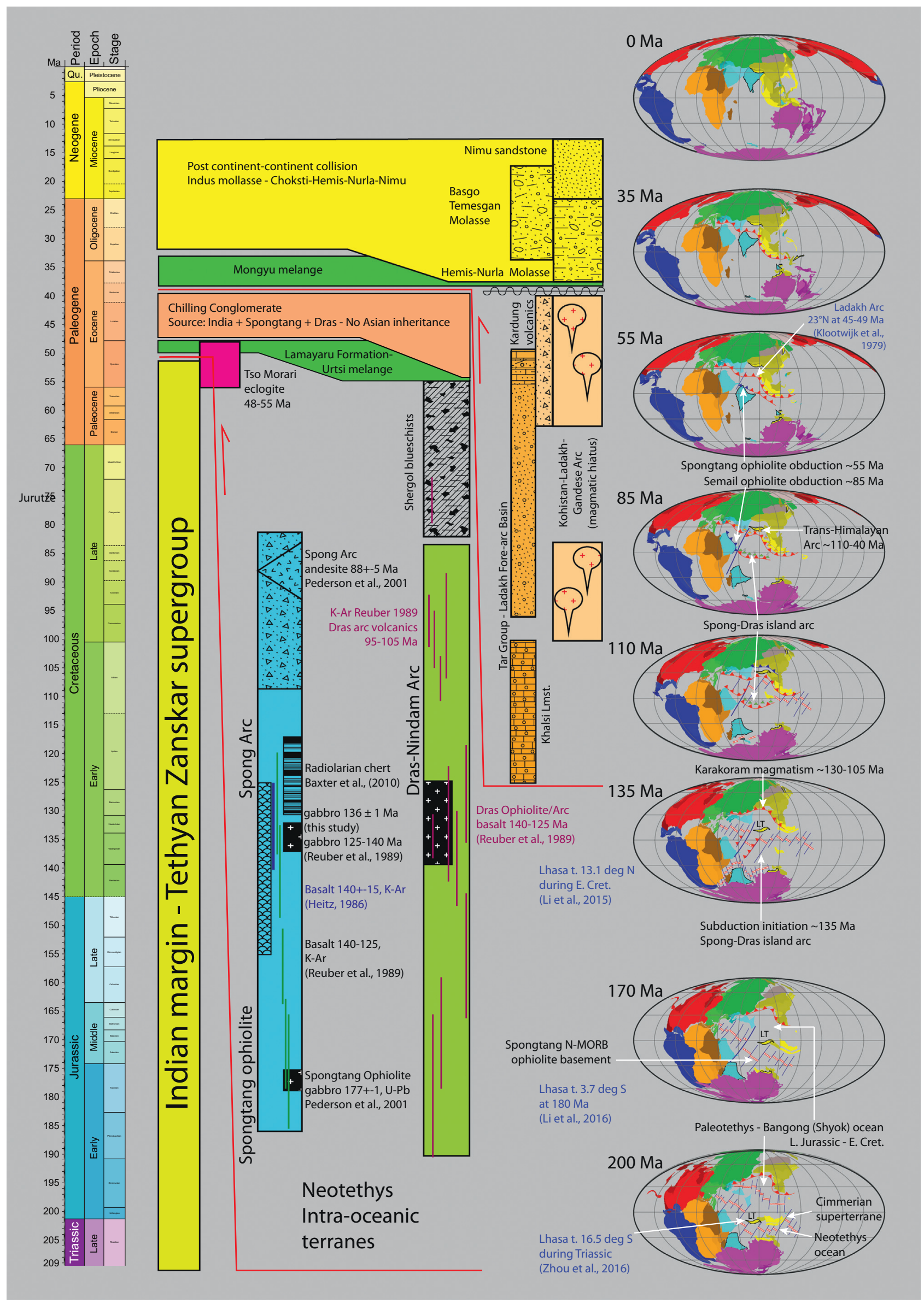


continent-continent collision of India with Eurasia during the Late Cretaceous ( $70 \mathrm{Ma})$. In their model, the Spongtang Massif does not represent the fore-arc basement of the continental margin of Eurasia (Ladakh Batholith) and is a separate subduction system to the intra-oceanic Dras-Kohistan Arc to the north, which first collides with the TransHimalayan continental arc of southern Eurasia before final continental collision of India and Eurasia. The Late Cretaceous ( $70 \mathrm{Ma})$ timing of obduction of the Spongtang Massif onto India is proposed largely to fit with the long-held consensus that the India-Asia continental collision began at $55 \mathrm{Ma}$ (de Sigoyer et al., 2000; Hu et al., 2016) and therefore, Eocene marine sediments ( $<55 \mathrm{Ma}$ ) could not have initially been overthrust by the ophiolite because they post-date the collision age. This model was disputed by Garzanti et al. (2005) on the basis of a lack of evidence for late thrusting. Also, the report of possible Late Eocene cherts within the mélange of the Lamayaru Complex immediately below the ophiolite by Colchen et al. (1987) is difficult to reconcile with the development of a small, shallow marine basins evolving adjacent to an ophiolite that was obducted onto India during the Late Cretaceous. We prefer the simplest explanation that the lower Eocene cherts within the mélange at the base of the Spongtang Massif and the lower Eocene Kong slate and Chulung la Formation below the ophiolite thrust sheet indicate post-early Eocene ophiolite obduction onto the Indian margin.

\subsection{Paleogeography and subduction initiation within Neotethys}

There is mounting evidence that all of the ophiolites along the IYTS formed within the Neotethyan Ocean before being obducted onto the Indian margin prior to final continental collision (Aitchison et al., 2007a). Thus, the position of the Lhasa terrane relative to India, Eurasia and the active arcs including the Kohistan-Ladakh-Gangdese and the Karakorum batholiths is important when interpreting where intra-oceanic terranes were situated before being accreted onto either India or Asia (Fig. 10). Recent paleomagnetic data indicate that the Lhasa terrane originated as a rifted portion of northern Gondwana (India or Australia) and was situated at about $16.5^{\circ}$ south throughout the Triassic (Zhou et al., 2016). It drifted northwards to $3.7^{\circ}$ south of the equator at $180 \mathrm{Ma}$ ( $\mathrm{Li}$ et al., 2016) and docked with the southern margin of Eurasia at about $25^{\circ} \mathrm{N}$ during the Late Cretaceous as constrained by the youngest (Lower Cretaceous) cherts within the Bangong-Nujiang Suture (Baxter et al., 2009). At 45-49 Ma the Ladakh Arc in the western Neotethyan ocean was at $23^{\circ} \mathrm{N}$ (Klootwijk et al., 1979), which marks the position of the southern margin of Eurasia. In contrast, the northern margin of India was only at $\sim 10^{\circ} \mathrm{N}$ around $50 \mathrm{Ma}$, see Acton (1999) and discussions by Aitchison et al. (2007a), indicating about $1500 \mathrm{~km}$ of ocean separated the northern passive margin of India and the southern convergent margin of Eurasia. This is consistent with the collision and obduction of the intraoceanic Spongtang Massif with India at about $55 \mathrm{Ma}$ in a separate event prior to final continental collision with Eurasia.

We follow the suggestion of Aitchison et al. (2012) that subduction may have initiated along major NNE-SSW trending transform faults that developed as the Lhasa terrane rifted away from Gondwana from Triassic to Jurassic (Fig. 10). The orientation of major transform faults in the Neotethys can be constrained by the relative movement (rifting) of Lhasa terrane from Gondwana starting in the Early Permian, that is, they must be parallel to relative plate movement and perpendicular to the rift, as shown in tectonic reconstructions by Stampfli and Borel (2002), Heine et al. (2004), Zahirovic et al. (2012) and Gibbons et al. (2015). The juxtaposition of old and new ocean crust at transform faults has the potential of initiating spontaneous subduction via the process of transform collapse (Stern, 2004). Similar rotations of subduction systems are documented in the Izu-Bonin-Marianas system (Hall, 2002) which originates down near Papua New Guinea then rotates $>90^{\circ}$ to its present positon. The "subduction initiation rule" proposed by Whattam and Stern (2011) predicts that supra-subduction zone (forearc) ophiolites display a sequence of igneous rocks that transition from fertile mantle-derived decompressional melts to increasingly metasomatised depleted mantle melts of boninitic composition to typical calc-alkaline arc magmas. The transition from Jurassic N-MORB Spongtang ophiolite to the Early Cretaceous calc-alkaline Spong Arc displays characteristics similar to the "subduction initiation rule". Thus, the Spongtang Massif has the potential to answer key questions including, A) the time it takes to initiate subduction, B) how initial fore-arc magmatism transitions to arc-like magmatism, C) how long arc magmatism persists and D) how intra-oceanic terranes are emplaced and preserved onto continental margins. Initiation of the Spong arc appears to involve an abrupt pulse of mafic magmatism at 136 Ma, which is about 10 m.y. older than most ophiolites along the Yarlung-Tsangpo suture in Tibet, suggesting that subduction may have initiated in the west and migrated eastward along the length of the transform fault. Supra-subduction zone volcanism occurred sporadically between $136 \mathrm{Ma}$ and $88 \mathrm{Ma}$ (Pedersen et al., 2001). However, in order to accommodate plate convergence, subduction and arc volcanism may have continued until final collision with India at $\sim 55 \mathrm{Ma}$. The youngest detrital zircon population in Kong Slate and Chulung la Formation is $\sim 53 \mathrm{Ma}$ (Najman et al., 2017) suggesting that the youngest sedimentary units on the Indian passive margin may have been receiving zircons from the active Spong Arc right up until final collision. This suggests that the Spong Arc was active for some 80 million years as an intra-oceanic island arc within the Neotethys before collision with India, which is consistent with the timescales of oceanic island arc development in the IzuBonin-Marianas system as outlined by Ishizuka et al. (2011).

The Semail Ophiolite in Oman shows a similar geochemical evolution from N-MORB to supra-subduction (fore-arc, boninitic) magmas but it did not evolve into a well-developed calc-alkaline island arc. This is probably due to the short period of time between formation at 95 Ma and obduction onto the Arabian continental margin at $85 \mathrm{Ma}$ (Searle et al., 2003). To the NW, along the Iraqi segment of the Zagros Suture Zone, ophiolites such as Hasanbag ophiolite complex have an almost identical age to the Semail ophiolite (92 Ma; Ali et al. (2012)), while intraoceanic terranes such as Walash-Naopurdan island arc are as young as 24 Ma (Ali et al., 2013) indicating continental collision started $<24$ million years ago in this region. The Semail and other ophiolites along the Zagros Suture Zone cannot be direct correlatives of the IYTS ophiolites because they were initiated along different subduction zones at different times and they formed either side of the Owen Fracture Zone (Gaina et al., 2015), which separated the rapidly northward moving Indian Plate from the slow-moving Arabian plate (Fig. 10). However, they do represent the last Neotethyan ophiolites to be obducted onto the leading edge of a passive continental margin in events before and separate to terminal continental collision. At Oman, terminal continental collision has yet to occur with a small amount of ocean floor yet to subduct beneath the active continental Makran Arc along the southern margin of Iran. Oman is a good example of an intra-oceanic ophiolite-arc complex colliding and obducting onto a continental margin long before final continental collision and is analogous to the collision of the Spongtang Massif with India well before terminal continental collision.

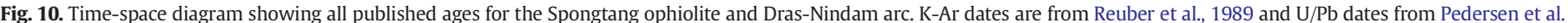

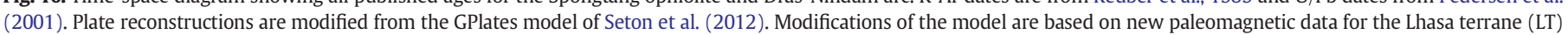

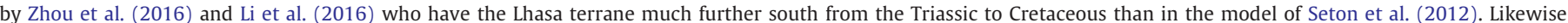

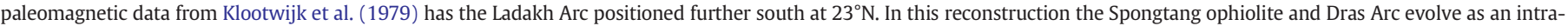

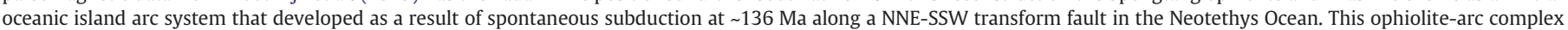

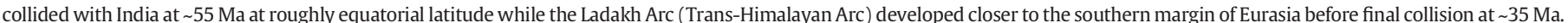




\section{Conclusions}

1) Zircons extracted from gabbro and leucogabbro from the Spongtang Massif both yield U-Pb SHRIMP ages of 136 and $133 \mathrm{Ma}$ (Early Cretaceous). This is younger than previous 177 Ma zircon (TIMS) ages reported by Pedersen et al. (2001).

2) Petrology and geochronology of the gabbro and basalts indicates two distinct mafic magmas are present - 1) N-MORB type volcanic rocks and minor gabbros of $\sim 177 \mathrm{Ma}$ age, 2) island-arc basalts, dolerite dykes and gabbros intruded through and onto the N-MORB volcanic pile. The older Jurassic crust represents the original Neotethys MORB-like crust while the Early Cretaceous gabbros and basalts represent renewed spreading associated with subduction initiation giving rise to the Spong Arc.

3) The depleted mantle-like, highly positive initial $\varepsilon_{\mathrm{Hf}}$ values indicate a purely intra-oceanic setting remote from continental crustal influence. The original Neotethyan Spongtang ophiolitic substrate is $\sim 177 \mathrm{Ma}$ and our acquired $\sim 136 \mathrm{Ma}$ zircon ages of leucogabbro represent intra-oceanic subduction initiation responsible for development of the Spong Arc before its emplacement onto the Indian passive margin as the Spongtang Massif.

4) The timing of collision is constrained by the age of the youngest Indian sedimentary units over which the ophiolite has been obducted. These are the Eocene Kong Slates and Chulung la Formation, which contain detrital zircons as young as $53 \mathrm{Ma}$ (Najman et al., 2017) and are likely to be derived from the final pulses of igneous activity within the Spong Arc before arc-continent collision extinguished any further igneous activity on the leading edge of India.

Supplementary data to this article can be found online at https://doi. org/10.1016/j.gr.2018.07.003.

\section{Acknowledgments}

This project was partially supported by the GeoQuEST Research Centre and a Small Project Grant (2017) of the University of Wollongong. We thank Jigmet Punchok for his assistance in the field. Shane Paxton (Australian National University) is thanked for mineral separation. Les Kinsley (Australian National University) is thanked for technical assistance with the zircon Lu-Hf analyses. We thank Paul Carr for undertaking the XRF analysis and Keira Webb for sample preparation.

\section{Appendix 1. XRF analytical methods}

Nine basalt and eight gabbro samples were collected from the Spongtang ophiolite within the Photang Valley. Samples were crushed using a TEMA chromium ring mill. Fused buttons were made for X-ray fluorescence (XRF) major element analysis. Depending on elemental concentrations estimated in trace element analysis, different types of flux were used. Pure metaborate was used for high silica samples, 57\% tetraborate to $43 \%$ metaborate was used for ultramafic samples, and $12 \%$ tetraborate to $22 \%$ metaborate was used for mafic samples. $400 \mathrm{mg}$ of sample was added to each flux ( $300 \mathrm{mg}$ for pure metaborate). Samples JP22, JP35 and JP44 were oxidized before being fused in the furnace by adding $5 \mathrm{ml}$ of lithium nitrate solution and left at $60{ }^{\circ} \mathrm{C}$ overnight. Pressed pellets for trace element analysis were created by mixing $\sim 5 \mathrm{~g}$ of sample with a polyvinyl acetate (PVA) binder and pressed into an aluminium cup using a hydraulic hand press. Trace element pressed pellets were then oven dried at $60^{\circ} \mathrm{C}$ for $12 \mathrm{~h}$. Whole rock geochemical analysis was conducted using a SPECTRO XEPOS energy dispersive polarization X-ray fluorescence spectrometer at the University of Wollongong. Additional trace elements and the rare-earth element analyses were undertaken at Australian Laboratory Services (ALS) at Brisbane, Australia using inductively coupled plasma-mass spectroscopy (ICP-MS).

\section{Appendix 2. SHRIMP U-Pb analytical method and data appraisal}

Heavy minerals were concentrated using heavy liquid and isodynamic separation techniques at the mineral separation laboratory of the Research School of Earth Sciences, the Australian National University (ANU). Using a binocular microscope, the concentrates were handpicked, and the chosen grains were cast into an epoxy resin disc along with reference Temora zircons (Black et al., 2003). After the epoxy cured, the mount was ground to a mid-section level through the grains and then polished. Cathodoluminescence $(\mathrm{CL})$ imaging was used to document the grains.

$\mathrm{U}-\mathrm{Th}-\mathrm{Pb}$ analyses of the zircons were undertaken on the Hiroshima University SHRIMP 2 instrument (now moved to Nagoya University) following analytical protocols of Williams (1998), with the raw data being reduced using ANU software 'PRAWN' and 'Lead'. Measurements of ${ }^{206} \mathrm{~Pb} /{ }^{238} \mathrm{U}$ in unknown zircons were calibrated using the Temora standard (U-Pb ages concordant at $417 \mathrm{Ma}$; Black et al., 2003). The reference zircon SL13 $(U=238 \mathrm{ppm})$ located in a set-up mount was used to calibrate $U$ and $T h$ abundance in the unknown zircons. The ISOPLOT program (Ludwig, 2003) was used to assess and plot the reduced and calibrated data. The results are plotted in a Tera-Wasserburg Concordia diagram prior to correction for common $\mathrm{Pb}$. The reason for plotting them without correction for common $\mathrm{Pb}$ is to demonstrate that given the small amount of common $\mathrm{Pb}$ in these zircons, the data already have close to concordant $\mathrm{U}-\mathrm{Pb}$ ages, prior to correction. Weighted mean ${ }^{206} \mathrm{~Pb} /{ }^{238} \mathrm{U}$ ages were calculated following the '207' method of Compston et al. (1984) and using the Cumming and Richards (1975) $\mathrm{Pb}$ composition for the likely age of the zircons.

\section{Appendix 3. LA-MC-ICP-MS zircon Lu-Hf isotopes}

Zircon hafnium isotopic compositions were determined over a single analytical session 5-6 May 2016, using the RSES ThermoFinnigan Neptune multi-collector ICPMS coupled to a Lambda Physik ArF, $193 \mathrm{~nm}$ excimer laser system with a 'HelEx' sample cell, following methods described by Hiess et al. (2009). Hf isotope analytical sites coincided with the U-Pb age determination sites where available. NIST 612 glass and a large zircon crystal from the Monastery kimberlite were used to initially tune the mass spectrometer to optimum sensitivity, adjust peak shape and ensure good collector positioning for all isotopes to be measured including those of $\mathrm{Lu}$ and $\mathrm{Yb}$ in addition to $\mathrm{Hf}$. Analyses were made with a $47 \mu \mathrm{m}$ diameter circular spot firing at $5 \mathrm{~Hz}$ with an energy density at the sample surface of $\sim 10 \mathrm{~J} / \mathrm{cm} 2$. For small zircons the spot overlapped onto the epoxy, but there was no detectable blank contribution. ${ }^{171} \mathrm{Yb}$, ${ }^{173} \mathrm{Yb},{ }^{174} \mathrm{Hf},{ }^{175} \mathrm{Lu},{ }^{176} \mathrm{Hf},{ }^{177} \mathrm{Hf},{ }^{178} \mathrm{Hf},{ }^{179} \mathrm{Hf}$ and ${ }^{181} \mathrm{Ta}$ isotopes were simultaneously measured in static-collection mode on 9 Faraday cups with $10^{11} \Omega$ resistors. Analysis of a gas blank and a suite of 6 secondary reference zircons with a range of ${ }^{176} \mathrm{Hf} /{ }^{177} \mathrm{Hf},{ }^{176} \mathrm{Lu} /{ }^{177} \mathrm{Hf}$ ratios ${ }^{176} \mathrm{Yb} /{ }^{177} \mathrm{Hf}$ (Monastery, Mud Tank, Plesovice, QGNG, R33 and FC1) were performed systematically after every 10-12 sample spot analyses throughout the session.

Data were acquired in $1 \mathrm{~s}$ integrations over $60 \mathrm{~s}$ or until the grain burned through. Time slices were later cropped to periods maintaining steady ${ }^{176} \mathrm{Hf} /{ }^{177} \mathrm{Hf}$ signals during data reduction on a custom Excel ${ }^{\mathrm{TM}}$ spreadsheet. Total Hf signal intensity typically fell from $>10$ to $\sim 6 \mathrm{~V}$ during a single analysis. The measured ${ }^{176} \mathrm{Lu} /{ }^{177} \mathrm{Hf},{ }^{176} \mathrm{Hf} /{ }^{177} \mathrm{Hf},{ }^{178} \mathrm{Hf} /{ }^{177} \mathrm{Hf}$ and ${ }^{174} \mathrm{Hf} /{ }^{177} \mathrm{Hf}$ ratios for all standard and sample analyses are presented in Supplementary Table 3. Mass bias was corrected using an exponential law (Chu et al., 2002; Russell et al., 1978; Woodhead et al., 2004) and a composition for ${ }^{179} \mathrm{Hf} /{ }^{177} \mathrm{Hf}$ of 0.732500 (Patchett et al., 1982). Yb and Lu mass bias factors were assumed to be identical and normalized using an exponential correction referenced to a ${ }^{173} \mathrm{Yb} /{ }^{171} \mathrm{Yb}$ ratio of 1.129197 (Vervoort et al., 2004). The intensity of the ${ }^{176} \mathrm{Hf}$ peak was determined accurately by removing isobaric interferences from ${ }^{176} \mathrm{Lu}$ and ${ }^{176} \mathrm{Yb}$. Interference-free ${ }^{175} \mathrm{Lu}$ and ${ }^{173} \mathrm{Yb}$ were measured and the interference peaks subtracted according to reported 
${ }^{176} \mathrm{Lu} /{ }^{175} \mathrm{Lu}$ and ${ }^{176} \mathrm{Yb} /{ }^{173} \mathrm{Yb}$ isotopic abundances of Vervoort et al. (2004). As a quality check of this procedure, ${ }^{178} \mathrm{Hf} /{ }^{177} \mathrm{Hf}$ and ${ }^{174} \mathrm{Hf} /{ }^{177} \mathrm{Hf}$ ratios for all zircon reference materials and samples were monitored. In particular the ${ }^{174} \mathrm{Hf} /{ }^{177} \mathrm{Hf}$ ratio is a sensitive monitor of the efficacy of $\mathrm{Yb}$ corrections owing to the relatively large ${ }^{174} \mathrm{Yb}$ isotopic interference (31.83\% abundance) that must be removed from the small ${ }^{174} \mathrm{Hf}$ isotope (0.16\% abundance). The mean ${ }^{178} \mathrm{Hf} /{ }^{177} \mathrm{Hf}$ and ${ }^{174} \mathrm{Hf} /{ }^{177} \mathrm{Hf}$ ratios for all standards and samples lie within uncertainty of the values published by Thirlwall and Anczkiewicz (2004) and Vervoort et al. (2004); Supplementary Table 3, regardless of zircon REE content demonstrating the accuracy of $\mathrm{Yb}$ corrections. Furthermore, no correlation exists between ${ }^{176} \mathrm{Hf} /{ }^{177} \mathrm{Hf}$ and ${ }^{178} \mathrm{Hf} /{ }^{177} \mathrm{Hf},{ }^{174} \mathrm{Hf} /{ }^{177} \mathrm{Hf},{ }^{176} \mathrm{Lu} /{ }^{177} \mathrm{Hf}$ ratios for any zircon reference materials, including higher $\mathrm{Lu} / \mathrm{Hf}$ standards FC1 and R33. Based on results from the six reference zircons, which were all within error of accepted solution values reported by Woodhead et al. (2004), no additional external corrections to the measured ${ }^{176} \mathrm{Hf} /{ }^{177} \mathrm{Hf}$ were required.

Zircon ${ }^{176} \mathrm{Lu} /{ }^{177} \mathrm{Hf}$ ratios must be accurately determined by LA-MCICPMS, to enable corrections for in-growth of radiogenic ${ }^{176} \mathrm{Hf}$. Average measured ${ }^{176} \mathrm{Lu} /{ }^{177} \mathrm{Hf}$ ratios within reference zircons with a range of reported compositions based on solution analyses are in good agreement with the measured laser analyses The mean ${ }^{176} \mathrm{Hf} /{ }^{177} \mathrm{Hf}$ ratios for the six reference zircons deviate from published solution values by +0.0 (Plesovice); +0.30 (Mud Tank), +0.33 (QGNG), +0.15 (FC-1), -0.24 (TEM-2), -0.16 (R33) and -0.08 (Monastery) $\varepsilon_{\mathrm{Hf}}$ units (Supplementary Table 3 ).

The segmental processing of the laser ablation data means that any down-hole variation in $\mathrm{Lu} / \mathrm{Hf}$ and ${ }^{176} \mathrm{Hf} /{ }^{177} \mathrm{Hf}$ ratio can be detected and tracked. In all sample spot analyses Lu/Hf and ${ }^{176} \mathrm{Hf} /{ }^{177} \mathrm{Hf}$ ratios were uniform throughout data acquisition.

\section{References}

Abrajevitch, A.V., Ali, J.R., Aitchison, J.C., Badengzhu, Davis, A.M., Liu, J.B., Ziabrev, S.V., 2005. Neotethys and the India-Asia collision: insights from a palaeomagnetic study of the Dazhuqu ophiolite, southern Tibet. Earth and Planetary Science Letters 233, 87-102.

Acton, G.D., 1999. Apparent polar wander of India since the Cretaceous with implications for regional tectonics and true polar wander. In: Radhakrishna, T., Piper, J.D.A. (Eds.) The Indian Subcontinent and Gondwana: A Palaeomagnetic and Rock Magnetic Perspective. Geological Society of India, Bangalore, pp. 129-175.

Ahmad, T., Tanaka, T., Sachan, H.K., Asahara, Y., Islam, R., Khanna, P.P., 2008. Geochemical and isotopic constraints on the age and origin of the Nidar Ophiolitic Complex, Ladakh, India: implications for the Neo-Tethyan subduction along the Indus suture zone. Tectonophysics 451, 206-224

Aitchison, J.C., Buckman, S., 2012. Accordion vs. quantum tectonics: insights into continental growth processes from the Paleozoic of eastern Gondwana. Gondwana Research 22, 674-680.

Aitchison, J.C., Clarke, G.L., Meffre, S., Cluzel, D., 1995. Eocene arc-continent collision in New-Caledonia and implications for regional southwest Pacific tectonic evolution. Geology 23, 161-164.

Aitchison, J.C., Badengzhu, Davis, A.M., Liu, J.B., Luo, H., Malpas, J.G., McDermid, I.R.C., Wu H.Y. Ziabrev, S.V., Zhou, M.F. 2000. Remnants of a Cretaceous intra-oceanic subduction system within the Yarlung-Zangbo suture (southern Tibet). Earth and Planetary Science Letters 183, 231-244

Aitchison, J.C., Davis, A.M., Badengzhu, B., Luo, H., 2002. New constraints on the India-Asia collision: the Lower Miocene Gangrinboche conglomerates, Yarlung Tsangpo suture zone, SE Tibet. Journal of Asian Earth Sciences 21, 251-263.

Aitchison, J.C., Davis, A.M., Abrajevitch, A.V., Ali, J.R., Badengzhu, Liu, J., Luo, H., McDermid, I.R.C., Zyabrev, S.V., 2003. Stratigraphic and sedimentological constraints on the age and tectonic evolution of the Neotethyan ophiolites along the Yarlung Tsangpo suture zone, Tibet. In: Dilek, Y., Robinson, P.T. (Eds.), Ophiolites in Earth History, pp. 147-164.

Aitchison, J.C., Ali, J.R., Davis, A.M., 2007a. When and where did India and Asia collide? Journal of Geophysical Research 112.

Aitchison, J.C., McDermid, I.R.C., Ali, J.R., Davis, A.M., Zyabrev, S.V., 2007b. Shoshonites in southern Tibet record Late Jurassic rifting of a Tethyan intraoceanic island arc. The Journal of Geology 115, 197-213.

Aitchison, J.C., Xia, X.P., Baxter, A.T., Ali, J.R., 2011. Detrital zircon U-Pb ages along the Yarlung-Tsangpo suture zone, Tibet: implications for oblique convergence and collision between India and Asia. Gondwana Research 20,691-709.

Aitchison, J., Gibbons, A., Müller, R., Whittaker, J., 2012. Birth of a Neotethyan intraoceanic arc. AGU Fall Meeting Abstracts.

Ali, S.A., Buckman, S., Aswad, K.J., Jones, B.G., Ismail, S.A., Nutman, A.P., 2012. Recognition of Late Cretaceous Hasanbag ophiolite-arc rocks in the Kurdistan Region of the Iraqi
Zagros suture zone: a missing link in the paleogeography of the closing Neotethys Ocean. Lithosphere 4, 395-410.

Ali, S.A., Buckman, S., Aswad, K.J., Jones, B.G., Ismail, S.A., Nutman, A.P., 2013. The tectonic evolution of a Neo-Tethyan (Eocene-Oligocene) island-arc (Walash and Naopurdan groups) in the Kurdistan region of the Northeast Iraqi Zagros Suture Zone. Island Arc 22, 104-125.

Ballard, J.R., Palin, J.M., Campbell, I.H., 2002. Relative oxidation states of magmas inferred from $\mathrm{Ce}(\mathrm{IV}) / \mathrm{Ce}(\mathrm{III})$ in zircon: application to porphyry copper deposits of northern Chile. Contributions to Mineralogy and Petrology 144, 347-364.

Bassoullet, J.P., Colchen, M., Juteau, T., Marcoux, J., Mascle, G., 1980. Structure of the Zanskar Nappes, Ladakh, Himalaya. Comptes Rendus Hebdomadaires Des Seances De L Academie Des Sciences Serie D 290, 389-392.

Baud, A., Arn, R., Bugnon, P., Crisinel, A., Dolivo, E., Escher, A., Hammerschlag, J., Marthaler, M., Masson, H., Steck, A., 1982. Le contact Gondwana-péri-Gondwana dans le Zanskar oriental (Ladakh, Himalaya). Bulletin de la Société Géologique de France 24, 341-361.

Baxter, A.T., Aitchison, J.C., Zyabrev, S.V., 2009. Radiolarian age constraints on Mesotethyan ocean evolution, and their implications for development of the Bangong-Nujiang suture, Tibet. Journal of the Geological Society of London 166, 689-694.

Baxter, A.T., Aitchison, J.C., Ali, J.R., Zyabrev, S.V., 2010. Early Cretaceous radiolarians from the Spongtang massif, Ladakh, NW India: implications for Neo-Tethyan evolution. Journal of the Geological Society of London 167, 511-517.

Baxter, A.T., Aitchison, J.C., Ali, J.R., Chan, J.S.L., Chan, G.H.N., 2016. Detrital chrome spinel evidence for a Neotethyan intra-oceanic island arc collision with India in the Paleocene. Journal of Asian Earth Sciences 128, 90-104.

Bédard, É., Hébert, R., Guilmette, C., Lesage, G., Wang, C.S., Dostal, J., 2009. Petrology and geochemistry of the Saga and Sangsang ophiolitic massifs, Yarlung Zangbo Suture Zone, Southern Tibet: evidence for an arc-back-arc origin. Lithos 113, 48-67.

Black, L.P., Kamo, S.L., Allen, C.M., Aleinikoff, J.N., Davis, D.W., Korsch, R.J., Foudoulis, C., 2003. TEMORA 1: a new zircon standard for Phanerozoic U-Pb geochronology. Chemical Geology 200, 155-170.

Borneman, N.L., Hodges, K.V., van Soest, M.C., Bohon, W., Wartho, J.-A., Cronk, S.S., Ahmad, T., 2015. Age and structure of the shyok suture in the ladakh region of northwestern India: Implications for slip on the karakoram fault system. Tectonics 34, 2011-2033.

Bouilhol, P., Schaltegger, U., Chiaradia, M., Ovtcharova, M., Stracke, A., Burg, J.P., Dawood, H., 2011. Timing of juvenile arc crust formation and evolution in the Sapat Complex (Kohistan-Pakistan). Chemical Geology 280, 243-256.

Bouilhol, P., Jagoutz, O., Hanchar, J.M., Dudas, F.O., 2013. Dating the India-Eurasia collision through arc magmatic records. Earth and Planetary Science Letters 366, 163-175.

Bouvier, A., Vervoort, J.D., Patchett, P.J., 2008. The Lu-Hf and Sm-Nd isotopic composition of CHUR: constraints from unequilibrated chondrites and implications for the bulk composition of terrestrial planets. Earth and Planetary Science Letters 273, 48-57.

Brookfield, M.E., Andrews-Speed, C.P., 1984. Sedimentology, petrography and tectonic significance of the shelf, flysch and molasse clastic deposits across the Indus suture zone, Ladakh, NW India. Sedimentary Geology 40, 249-286.

Buckman, S., Aitchison, J.C., 2004. Tectonic evolution of Paleozoic terranes in West Junggar, Xinjiang, northwest China. In: Malpas, J.G., Fletcher, C.J.N., Ali, J.R., Aitchison, J.C (Eds.), Aspects of the Tectonic Evolution of China. Geological Society of London, pp. 101-130.

Chu, N.C., Taylor, R.N., Chavagnac, V., Nesbitt, R.W., Boella, R.M., Milton, J.A., German, C.R., Bayon, G., Burton, K., 2002. Hf isotope ratio analysis using multi-collector inductively coupled plasma mass spectrometry: an evaluation of isobaric interference corrections. Journal of Analytical Atomic Spectrometry 17, 1567-1574.

Clift, P.D., Degnan, P.J., Hannigan, R., Blusztajn, J., 2000. Sedimentary and geochemical evolution of the Dras forearc basin, Indus suture, Ladakh Himalaya, India. Geological Society of America Bulletin 112, 450-466.

Clift, P.D., Shimizu, N., Layne, G.D., Blusztajn, J., 2001. Tracing patterns of erosion and drainage in the Paleogene Himalaya through ion probe $\mathrm{Pb}$ isotope analysis of detrital K-feldspars in the Indus Molasse, India. Earth and Planetary Science Letters 188, 475-491.

Clift, P., Hannigan, R., Blusztajn, J., Draut, A.E., 2002a. Geochemical evolution of the DrasKohistan Arc during collision with Eurasia: evidence from the Ladakh Himalaya, India. The Island Arc 11, 255-273.

Clift, P.D., Carter, A., Krol, M., Kirby, E., 2002b. Constraints on India-Eurasia collision in the Arabian Sea region taken from the Indus Group, Ladakh Himalaya, India. Tectonic and Climatic Evolution of the Arabian Sea Region 195, 97-116.

Colchen, M., Reuber, I., Bassoullet, J.P., Bellier, J.-P., Blondeau, A., Lys, M., De Wever, P., 1987. Donnees biostratigraphiques sur les melanges ophiolitiques du Zanskar, Himalaya du Ladakh (Biostratigraphic data on the Zanskar ophiolitic melanges, Ladakh-Himalaya (India)). Comptes Rendus de l'Academie des Sciences Serie II 305, 403-406.

Compston, W., Williams, I., Meyer, C., 1984. U-Pb geochronology of zircons from lunar breccia 73217 using a sensitive high mass-resolution ion microprobe. Journal of Geophysical Research - Solid Earth 89.

Corfield, R.I., Searle, M.P., 2000. Crustal shortening estimates across the north Indian continental margin, Ladakh, NW India. Tectonics of the Nanga Parbat Syntaxis and the Western Himalaya vol. 170, pp. 395-410.

Corfield, R.I., Searle, M.P., Green, O.R., 1999. Photang thrust sheet: an accretionary complex structurally below the Spontang ophiolite constraining timing and tectonic environment of ophiolite obduction, Ladakh Himalaya, NW India. Journal of the Geological Society of London 156, 1031-1044.

Corfield, R.I., Searle, M.P., Pedersen, R.B., 2001. Tectonic setting, origin, and obduction history of the spontang ophiolite, Ladakh Himalaya, NW India. Journal of Geology 109, 715-736. 
Coward, M.P., Butler, R.W.H., Khan, M.A., Knipe, R.J., 1987. The tectonic history of Kohistan and its implications for Himalayan structure. Journal of the Geological Society of London 144, 377-391.

Cumming, G.L., Richards, J.R., 1975. Ore lead isotope ratios in a continuously changing Earth. Earth and Planetary Science Letters 28, 155-171.

Davis, A.M., Aitchison, J.C., Hui, L., 2004. Conglomerates record the tectonic evolution of the Yarlung-Tsangpo suture zone in southern Tibet. Geological Society - Special Publications 226, 235-246.

de Sigoyer, J., Chavagnac, V., Blichert-Toft, J., Villa, I.M., Luais, B., Guillot, S., Cosca, M., Mascle, G., 2000. Dating the Indian continental subduction and collisional thickening in the northwest Himalaya: multichronology of the Tso Morari eclogites. Geology 28, 487.

de Sigoyer, J., Guillot, S., Dick, P., 2004. Exhumation of the ultrahigh-pressure Tso Morari unit in eastern Ladakh (NW Himalaya): a case study. Tectonics 23 (n/a-n/a).

Dewey, J.F., 2005. Orogeny can be very short. Proceedings of the National Academy of Sciences of the United States of America 102, 15286-15293.

Dewey, J.F., Cande, S., Pitman, W.C., 1989. Tectonic evolution of the India Eurasia collision zone. Eclogae Geologicae Helvetiae 82, 717-734.

Dhuime, B., Hawkesworth, C., Cawood, P., 2011. Geochemistry. When continents formed. Science 331, 154-155.

Dilek, Y., Furnes, H., 2011. Ophiolite genesis and global tectonics: geochemical and tectonic fingerprinting of ancient oceanic lithosphere. Geological Society of America Bulletin $123,387-411$

Donaldson, D.G., Webb, A.A.G., Menold, C.A., Kylander-Clark, A.R.C., Hacker, B.R., 2013. Petrochronology of Himalayan ultrahigh-pressure eclogite. Geology 41, 835-838.

Dubois-Cote, V., Hebert, R., Dupuis, C., Wang, C.S., Li, Y.L., Dostal, J., 2005. Petrological and geochemical evidence for the origin of the Yarlung Zangbo ophiolites, southern Tibet. Chemical Geology 214, 265-286.

Dunlap, W.J., Wysoczanski, R., 2002. Thermal evidence for early Cretaceous metamorphism in the Shyok suture zone and age of the Khardung volcanic rocks, Ladakh, India. Journal of Asian Earth Sciences 20, 481-490.

Dupuis, C., Hebert, R., Dubois-Cote, V., Guilmette, C., Wang, C.S., Li, Y.L., Li, Z.J., 2005 The Yarlung Zangbo Suture Zone ophiolitic melange (southern Tibet): new insights from geochemistry of ultramafic rocks. Journal of Asian Earth Sciences 25, 937-960.

Dürr, S.B., 1996. Provenance of Xigaze fore-arc basin clastic rocks (Cretaceous, South Tibet). Geological Society of America Bulletin 108, 669-684.

Ehiro, M., Kojima, S., Sato, T., Ahmad, T., Ohtani, T., 2007. Discovery of Jurassic ammonoids from the Shyok suture zone to the northeast of Chang La Pass, Ladakh, northwest India and its tectonic significance. Island Arc 16, 124-132.

Einsele, G., Liu, B., Dürr, S., Frisch, W., Liu, G., Luterbacher, H.P., Ratschbacher, L., Ricken, W., Wendt, J., Wetzel, A., Yu, G., Zheng, H., 1994. The Xigaze forearc basin: evolution and facies architecture (Cretaceous, Tibet). Sedimentary Geology 90, 1-32.

Frank, W., 1977. Geological observations in the Ladakh area (Himalayas). A preliminary report. Schweizerische Mineralogische und Petrographische Mitteilungen 57, 89-113.

Fuchs, G., 1979. On the geology of western Ladakh. Jahrbuch der Geologischen Bundesanstalt A 122, 513-540.

Fuchs, G., 1981. Outline of the geology of the Himalaya. Mitteilungen. Österreichische Geologische Gesellschaft 74/75, 101-127.

Fuchs, G., 1982. The geology of Western Zanskar. 125, 1-50.

Fuchs, G., 1986. Geology of the Markha-Khurnak Region in Ladakh (India). Jahrbuch der Geologischen Bundesanstalt A 128, 403-437.

Fuchs, G., Willems, H., 1990. The final stages of sedimentation in the Tethyan Zone of Zanskar and their geodynamic significance (Ladakh-Himalaya). Jahrbuch der Geologischen Bundesanstalt 133, 259-273.

Gaetani, M., Garzanti, E., 1991. Multicyclic history of the northern India continentalmargin (northwestern Himalaya). AAPG Bulletin-American Association of Petroleum Geologists 75, 1427-1446.

Gaina, C., van Hinsbergen, D.J.J., Spakman, W., 2015. Tectonic interactions between India and Arabia since the Jurassic reconstructed from marine geophysics, ophiolite geology, and seismic tomography. Tectonics 34, 875-906.

Gansser, A., 1964. The Geology of the Himalayas. Wiley-Interscience, New York (289 pp.).

Garzanti, E., Van Haver, T., 1988. The indus clastics: forearc basin sedimentation in the Ladakh Himalaya (India). Sedimentary Geology 59, 237-249.

Garzanti, E., Baud, A., Mascle, G., 1987. Sedimentary record of the northward flight of India and its collision with Eurasia (Ladakh Himalaya, India). Geodinamica Acta 1, 297-312.

Garzanti, E., Sciunnach, D., Gaetani, M., Corfield, R.I., Watts, A.B., Searle, M.P., 2005. Discussion on subsidence history of the north Indian continental margin, Zanskar-Ladakh Himalaya, NW India. Journal of the Geological Society of London 162, 889-892.

Gibbons, A.D., Zahirovic, S., Muller, R.D., Whittaker, J.M., Yatheesh, V., 2015. A tectonic model reconciling evidence for the collisions between India, Eurasia and intraoceanic arcs of the central-eastern Tethys. Gondwana Research 28, 451-492.

Girardeau, J., Marcoux, J., Yougong, Z., 1984. Lithologic and tectonic environment of the Xigaze ophiolite (Yarlung Zangbo suture zone, Southern Tibet, China), and kinematics of its emplacement. Eclogae Geologicae Helvetiae 77, 153-170.

Girardeau, J., Mercier, J.C.C., Yougong, Z., 1985a. Origin of the Xigaze ophiolite, Yarlung Zangbo suture zone, Southern Tibet. Tectonophysics 119, 407-433.

Girardeau, J., Mercier, J.C.C., Zao, Y.O., 1985b. Structure of the Xigaze ophiolite, Yarlung Zangbo suture zone, Southern Tibet, China - genetic-implications. Tectonics 4, 267.

Groppo, C., Rolfo, F., Sachan, H.K., Rai, S.K., 2016. Petrology of blueschist from the Western Himalaya (Ladakh, NW India): exploring the complex behavior of a lawsonitebearing system in a paleo-accretionary setting. Lithos $252,41-56$.
Hall, R., 2002. Cenozoic geological and plate tectonic evolution of SE Asia and the SW Pacific: computer-based reconstructions, model and animations. Journal of Asian Earth Sciences 20, 353-431.

Hébert, R., Bezard, R., Guilmette, C., Dostal, J., Wang, C.S., Liu, Z.F., 2012. The Indus-Yarlung Zangbo ophiolites from Nanga Parbat to Namche Barwa syntaxes, southern Tibet: first synthesis of petrology, geochemistry, and geochronology with incidences on geodynamic reconstructions of Neo-Tethys. Gondwana Research 22, 377-397.

Heine, C., Muller, R.D., Gaina, C., 2004. Reconstructing the Lost Eastern Tethys Ocean Basin: convergence history of the SE Asian margin and marine gateways. Geophysical Monograph Series 149, 37-54.

Heitz, A., 1986. Datations par méthode K/Ar sur filons du massif ophiolitique de Spongtang, rapport Strasbourg. p. 28.

Henderson, A.L., Najman, Y., Parrish, R., BouDagher-Fadel, M., Barford, D., Garzanti, E. Ando, S., 2010. Geology of the Cenozoic Indus Basin sedimentary rocks: paleoenvironmental interpretation of sedimentation from the western Himalaya during the early phases of India-Eurasia collision. Tectonics 29.

Henderson, A.L., Najman, Y., Parrish, R., Mark, D.F., Foster, G.L., 2011. Constraints to the timing of India-Eurasia collision; a re-evaluation of evidence from the Indus Basin sedimentary rocks of the Indus-Tsangpo Suture Zone, Ladakh, India. Earth-Science Reviews 106, 265-292.

Heri, A.R., Aitchison, J.C., King, J.A., Villa, I.M., 2015. Geochronology and isotope geochemistry of Eocene dykes intruding the Ladakh Batholith. Lithos 212-215, 111-121.

Hiess, J., Bennett, V.C., Nutman, A.P., Williams, I.S., 2009. In situ U-Pb, O and Hf isotopic compositions of zircon and olivine from Eoarchaean rocks, West Greenland: new insights to making old crust. Geochimica et Cosmochimica Acta 73, 4489-4516.

Hole, M.J., Saunders, A.D., Marriner, G.F., Tarney, J., 1984. Subduction of pelagic sediments: implications for the origins of Ce-anomolous basalts from the Marianas islands. Journal of the Geological Society, London 141, 453-472.

Holm, R.J., Spandler, C., Richards, S.W., 2015. Continental collision, orogenesis and arc magmatism of the Miocene Maramuni arc, Papua New Guinea. Gondwana Research 28, 1117-1136.

Honegger, K., Dietrich, V., Frank, W., Gansser, A., Thoni, M., Trommsdorff, V., 1982. Magmatism and Metamorphism in the Ladakh Himalayas (the Indus-Tsangpo Suture Zone). Earth and Planetary Science Letters 60, 253-292.

Honegger, K., Le Fort, P., Mascle, G., Zimmermann, J.L., 1989. The blueschists along the Indus suture zone in Ladakh, NW Himalaya. Journal of Metamorphic Geology 7, 57-73.

Hu, X.M., Garzanti, E., Wang, J.G., Huang, W.T., An, W., Webb, A., 2016. The timing of IndiaAsia collision onset - facts, theories, controversies. Earth-Science Reviews 160, 264-299.

Huang, C.Y., Yuan, P.B., Lin, C.W., Wang, T.K., Chang, C.P., 2000. Geodynamic processes of Taiwan arc-continent collision and comparison with analogs in Timor, Papua New Guinea, Urals and Corsica. Tectonophysics 325, 1-21.

Huang, C.Y., Chien, C.W., Yao, B., Chang, C.P., 2008. The Lichi Mélange: a collision mélange formation along early arcward backthrusts during forearc basin closure, Taiwan arccontinent collision. In: Draut, A.E., Clift, P.D., Scholl, D.W. (Eds.), Formation and Applications of the Sedimentary Record in Arc Collision Zones, pp. 127-154.

Irvine, T.N., Baragar, W.R.A., 1971. A guide to the chemical classification of the common volcanic rocks. Canadian Journal of Earth Sciences 8, 523-548.

Ishizuka, O., Tani, K., Reagan, M.K., Kanayama, K., Umino, S., Harigane, Y., Sakamoto, I., Miyajima, Y., Yuasa, M., Dunkley, D.J., 2011. The timescales of subduction initiation and subsequent evolution of an oceanic island arc. Earth and Planetary Science Letters 306, 229-240

Ishizuka, O., Hickey-Vargas, R., Arculus, R.J., Yogodzinski, G.M., Savov, I.P., Kusano, Y., McCarthy, A., Brandl, P.A., Sudo, M., 2018. Age of Izu-Bonin-Mariana arc basement. Earth and Planetary Science Letters 481, 80-90.

Jan, M.Q., Windley, B.F., Khan, A., 1985. The Waziristan ophiolite, Pakistan - general geology and chemistry of chromite and associated phases. Economic Geology 80, 294-306.

Ji, W.Q., Wu, F.Y., Chung, S.L., Li, J.X., Liu, C.Z., 2009. Zircon U-Pb geochronology and Hf isotopic constraints on petrogenesis of the Gangdese batholith, southern Tibet. Chemical Geology 262, 229-245.

Jones, R.E., Kirstein, L.A., Kasemann, S.A., Dhuime, B., Elliott, T., Litvak, V.D., Alonso, R. Hinton, R., Facility, E.I.M., 2015. Geodynamic controls on the contamination of Cenozoic arc magmas in the southern Central Andes: insights from the $\mathrm{O}$ and Hf isotopic composition of zircon. Geochimica et Cosmochimica Acta 164, 386-402.

Kakar, M.I., Collins, A.S., Mahmood, K., Foden, J.D., Khan, M., 2012. U-Pb zircon crystallization age of the Muslim Bagh ophiolite: enigmatic remains of an extensive preHimalayan arc. Geology 40, 1099-1102.

Kelemen, P.B., Sonnenfeld, M.D., 1983. Stratigraphy, structure, petrology and local tectonics, central Ladakh, NW Himalaya. Schweizerische Mineralogische und Petrographische Mitteilungen 63, 267-287.

Khan, M.A., Jan, M.Q., Weaver, B.L., 1993. Evolution of the lower arc crust in Kohistan, N. Pakistan; temporal arc magmatism through early, mature and intra-arc rift stages. In: Treloar, P.J., Searle, M.P. (Eds.), Himalayan Tectonics. Geological Society of London, London, United Kingdom, pp. 123-138.

Klootwijk, C., Sharma, M.L., Gergan, J., Tirkey, B., Shah, S.K., Agarwal, V., 1979. The extent of Greater India, II. Palaeomagnetic data from the Ladakh Intrusives at Kargil, northwestern Himalayas. Earth and Planetary Science Letters 44, 47-64.

Kocak, K., Isik, F., Arslan, M., Zedef, V., 2005. Petrological and source region characteristics of ophiolitic hornblende gabbros from the Aksaray and Kayseri regions, central Anatolian crystalline complex, Turkey. Journal of Asian Earth Sciences 25, 883-891.

Kojima, S., Ahmad, T., Tanaka, T., Bagati, T.N., Mishra, M., Kumar, R., Islam, R., Khanna, P., 2001. Early Cretaceous radiolarians from the Indus suture zone, Ladakh, northern India. News of Osaka Micropaleontologists. 12, pp. 257-270.

Kumar, S., Bora, S., Sharma, U.K., 2016. Geological appraisal of Ladakh and Tirit granitoids in the Indus-Shyok Suture Zones of Northwest Himalaya, India. Journal of the Geological Society of India 87, 737-746. 
La Touche, D., 1888. Rediscovery of nummulites in Zanskar. Records of the Geological Survey of India 21, 160-162.

Leech, M.L., Singh, S., Jain, A.K., Klemperer, S.L., Manickavasagam, R.M., 2005. The onset of India-Asia continental collision: early, steep subduction required by the timing of UHP metamorphism in the western Himalaya. Earth and Planetary Science Letters 234 83-97.

Leech, M.L., Singh, S., Jain, A.K., 2007. Continuous metamorphic zircon growth and interpretation of U-Pb SHRIMP dating: an example from the western Himalaya. International Geology Review 49, 313-328.

Li, Z.Y., Ding, L., Lippert, P.C., Song, P.P., Yue, Y.H., van Hinsbergen, D.J.J., 2016. Paleomagnetic constraints on the Mesozoic drift of the Lhasa terrane (Tibet) from Gondwana to Eurasia. Geology 44, 727-730.

Liu, J.B., Aitchison, J.C., 2002. Upper Paleocene radiolarians from the Yamdrok melange south Xizang (Tibet), China. Micropaleontology 48, 145-154.

Liu, D., Huang, Q., Fan, S., Zhang, L., Shi, R., Ding, L., 2014. Subduction of the BangongNujiang Ocean: constraints from granites in the Bangong Co area, Tibet. Geological Journal 49, 188-206.

Ludwig, K.R., 2003. Isoplot 3.00. A Geochronological Toolkit for Microsoft Excel. vol. 4. Berkeley Geochronology Center, Berkeley, California (70 pp.).

Lydekker, R., 1880. Geology of Ladakh and neighbouring districts. Records of the Geological Survey of India 13, 26-59.

Lydekker, R., 1883. The geology of the Kashmir and Chamba territories and the districts of Khagan. Memoirs - Geological Survey of India 22, 211-224.

MacMahon, C., 1901. Petrological notes on some peridotites, serpentines, gabbros, and associated rocks, from Ladakh, north-western Himalaya. Mere. Geol. Soc. India 31, 303-329.

Mahéo, G., Bertrand, H., Guillot, S., Villa, I.M., Keller, F., Capiez, P., 2004. The South Ladakh ophiolites (NW Himalaya, India): an intra-oceanic tholeiitic arc origin with implication for the closure of the Neo-Tethys. Chemical Geology 203, 273-303.

Mahéo, G., Fayoux, X., Guillot, S., Garzanti, E., Capiez, P., Mascle, G., 2006. Relicts of an intraoceanic arc in the Sapi-Shergol mélange zone (Ladakh, NW Himalaya, India): implications for the closure of the Neo-Tethys Ocean. Journal of Asian Earth Sciences 26 , 695-707.

Malpas, J., Zhou, M.-F., Robinson, P.T., Reynolds, P.H., 2003. Geochemical and geochronological constraints on the origin and emplacement of the Yarlung Zangbo ophiolites, Southern Tibet. Geological Society, London, Special Publications 218, 191-206.

McDermid, IR.C., Aitchison, J.C. Davis, A.M., Harrison, T.M., Grove, M., 2002. The Zedong terrane: a Late Jurassic intra-oceanic magmatic arc within the Yarlung-Tsangpo suture zone, southeastern Tibet. Chemical Geology 187, 267-277.

Miller, C., Schuster, R., Klotzli, U., Frank, W., Purtscheller, F., 1999. Post-collisional potassic and ultrapotassic magmatism in SW Tibet: geochemical and $\mathrm{Sr}-\mathrm{Nd}-\mathrm{Pb}-\mathrm{O}$ isotopic constraints for mantle source characteristics and petrogenesis. Journal of Petrology 40, 1399-1424.

Miller, C., Thoni, M., Frank, W., Schuster, R., Melcher, F., Meisel, T, Zanetti, A., 2003. Geochemistry and tectonomagmatic affinity of the Yungbwa ophiolite, SW Tibet. Lithos $66,155-172$.

Miyashiro, A., 1973. The Troodos ophiolitic complex was probably formed in an island arc Earth and Planetary Science Letters 19, 218-224.

Miyashiro, A., 1975. Classification, characteristics, and origin of ophiolites. Journal of Geology 83, 249-281.

Molnar, P., Tapponnier, P., 1975. Cenozoic tectonics of Asia: effects of a continental collision: features of recent continental tectonics in Asia can be interpreted as results of the India-Eurasia collision. Science 189, 419-426.

Moores, E.M., Roeder, D.H., Abbas, S.G., Ahmad, Z., 1980. Geology and emplacement of the Muslim Bagh ophiolite complex. In: Panayiotou, A. (Ed.), Ophiolites; Proceedings, International ophiolite symposium. Cyprus Minist. Agric. Nat. Resour. Geol. Surv. Dep., Nicosia, Cyprus, pp. 424-429.

Najman, Y., Jenks, D., Godin, L., Boudagher-Fadel, M., Millar, I., Garzanti, E., Horstwood, M., Bracciali, L., 2017. The Tethyan Himalayan detrital record shows that India-Asia terminal collision occurred by $54 \mathrm{Ma}$ in the Western Himalaya. Earth and Planetary Science Letters 459, 301-310.

Nicolas, A., Girardeau, J., Marcoux, J., Dupre, B., Wang, X.B., Cao, Y.G., Zheng, H.X., Xiao, X.C. 1981. The Xigaze ophiolite (Tibet) - a peculiar oceanic lithosphere. Nature 294 414-417.

Paces, J.B., Miller, J.D., 1993. Precise U-Pb ages of Duluth complex and related mafic intrusions, northeastern Minnesota: geochronological insights to physical, petrogenetic, paleomagnetic, and tectonomagmatic processes associated with the $1.1 \mathrm{Ga}$ midcontinent rift system. Journal of Geophysical Research - Solid Earth 98, 13997-14013.

Pallister, J.S., Knight, R.J., 1981. Rare-earth element geochemistry of the Samail ophiolite near Ibra, Oman. In: Coleman Robert, G., Hopson Clifford, A. (Eds.), Oman Ophiolite. American Geophysical Union, Washington, DC, United States, pp. 2673-2697.

Patchett, P.J., Kouvo, O., Hedge, C.E., Tatsumoto, M., 1982. Evolution of continental crust and mantle heterogeneity: evidence from Hf isotopes. Contributions to Mineralogy and Petrology 78, 279-297.

Pearce, J.A., 1975. Basalt geochemistry used to investigate past tectonic environments on Cyprus. Tectonophysics 25, 41-67.

Pearce, J.A., 1982. Trace element characteristics of lavas from destructive plate boundaries. In: Thorpe, R.S. (Ed.), Orogenic Andesites and Related Rocks. John Wiley and Sons, Chichester, England, pp. 528-548.

Pearce, J.A., 2008. Geochemical fingerprinting of oceanic basalts with applications to ophiolite classification and the search for Archean oceanic crust. Lithos 100, 14-48.

Pearce, J.A., Cann, J.R., 1973. Tectonic setting of basic volcanic-rocks determined using trace-element analyses. Earth and Planetary Science Letters 19, 290-300.

Pearce, J.A., Robinson, P.T., 2010. The Troodos ophiolitic complex probably formed in a subduction initiation, slab edge setting. Gondwana Research 18, 60-81.

Pedersen, R.B., Searle, M.P., Corfield, R.I., 2001. U-Pb zircon ages from the Spontang Ophiolite, Ladakh Himalaya. Journal of the Geological Society of London 158, 513-520.
Ravikant, V., 2006. Utility of Rb-Sr geochronology in constraining Miocene and Cretaceous events in the eastern Karakoram, Ladakh, India. Journal of Asian Earth Sciences 27, 534-543.

Ravikant, V., Wu, F.Y., Ji, W.Q., 2009. Zircon U-Pb and Hf isotopic constraints on petrogenesis of the Cretaceous-Tertiary granites in eastern Karakoram and Ladakh, India. Lithos 110, 153-166.

Reibel, G., Reuber, I., 1982. The Peculiar Spongtang-Photaksar Ophiolitic Nappe (Ladakh, Himalaya). Comptes Rendus de L Academie des Sciences Serie II 294, 557.

Reichardt, H., Weinberg, R.F., Andersson, U.B., Fanning, C.M., 2010. Hybridization of granitic magmas in the source: the origin of the Karakoram Batholith, Ladakh, NW India. Lithos 116, 249-272.

Reuber, I., 1986. Geometry of accretion and oceanic thrusting of the Spongtang Ophiolite, Ladakh-Himalaya. Nature 321, 592-596.

Reuber, I., 1989. The Dras Arc - 2 successive volcanic events on eroded oceanic-crust. Tectonophysics 161, 93-106.

Reuber, I., 1990. The Shyok ophiolite in Ladakh-Himalaya: relics of the Tethyan oceanic crust overlain by volcanosedimentary arc series of mid-Cretaceous age. Comptes Rendus de l'Academie des Sciences Serie II 310, 1255-1262.

Reuber, I., Montigny, R., Thuizat, R., Heitz, A., 1989. K-Ar Ages of ophiolites and arc volcanics of the Indus suture zone - clues on the early evolution of the Neo-Tethys. Eclogae Geologicae Helvetiae 82, 699-715.

Reuber, I., Colchen, M., Mevel, C., 1992. The Spongtang ophiolite and ophiolitic melanges of the Zanskar, NW Himalaya, tracing the evolution of the closing Tethys in the Upper Cretaceous to the early Tertiary. In: Sinha, A.K. (Ed.), Himalayan Orogen and Global Tectonics. International Lithosphere Programme, Publication 197. Balkema, pp. 235-266.

Reuber, I., Colchen, M., Mevel, C., 2015. The geodynamic evolution of the South-Tethyan, margin in Zanskar, NW-Himalaya, as revealed by the Spongtang ophiolitic melanges. Geodinamica Acta 1, 283-296.

Rex, A.J., Searle, M.P., Tirrul, R., Crawford, M.B., Prior, DJ. Rex, D.C., Barnicoat, A.C., 1988. The geochemical and tectonic evolution of the central Karakoram, North Pakistan. In: Shackleton, R.M., Dewey, J.F., Windley, B.F. (Eds.), Tectonic Evolution of the Himalayas and Tibet. Royal Society of London, London, United Kingdom, pp. 229-255.

Robertson, A.H.F., Degnan, P.J., 1993. Sedimentology and tectonic implications of the Lamayuru Complex - deep-water facies of the Indian passive margin, Indus Suture Zone, Ladakh Himalaya. Himalayan Tectonics 74, 299-321.

Robertson, A., Degnan, P., 1994. The Dras arc complex - lithofacies and reconstruction of a Late Cretaceous oceanic volcanic arc in the Indus suture zone, Ladakh-Himalaya. Sedimentary Geology 92, 117-145.

Rolland, Y., 2002. From intra-oceanic convergence to post-collisional evolution: example of the India-Asia convergence in NW Himalaya, from Cretaceous to present. In: Rosenbaum, G., Lister, G.S. (Eds.), Journal of the Virtual Explorer, pp. 185-208.

Russell, W., Papanastassiou, D., Tombrello, T., 1978. Ca isotope fractionation on the Earth and other solar system materials. Geochimica et Cosmochimica Acta 42, 1075-1090.

Ryan, W.B.F., Carbotte, S.M., Coplan, J.O., O'Hara, S., Melkonian, A., Arko, R., Weissel, R.A., Ferrini, V., Goodwillie, A., Nitsche, F., Bonczkowski, J., Zemsky, R., 2009. Global multi-resolution topography synthesis. Geochemistry, Geophysics, Geosystems 10 (n/a-n/a)

Sarwar, G., 1992. Tectonic setting of the Bela Ophiolites, southern Pakistan. Tectonophysics 207, 359-381.

Searle, M.P., 1986. Structural evolution and sequence of thrusting in the High Himalayan, Tibetan-Tethys and Indus suture zones of Zanskar and Ladakh, Western Himalaya. Journal of Structural Geology 8, 923.

Searle, M., Cox, J., 1999. Tectonic setting, origin, and obduction of the Oman ophiolite. Geological Society of America Bulletin 111, 104-122.

Searle, M.P., Tirrul, R., 1991. Structural and thermal evolution of the Karakoram crust. Journal of the Geological Society, London 148, 65-82.

Searle, M.P., Cooper, D.J.W., Rex, A.J., 1988. Collision tectonics of the Ladakh Zanskar Himalaya. Philosophical Transactions of the Royal Society A: Mathematical, Physical and Engineering Sciences 326, 117.

Searle, M.P., Parrish, R.R., Tirrul, R., Rex, D.C., 1990a. Age of crystallization and cooling of the K2 gneiss in the Baltoro Karakoram. Journal of the Geological Society, London 147, 603-606.

Searle, M.P., Pickering, K.T., Cooper, D.J.W., 1990b. Restoration and evolution of the intermontane Indus molasse basin, Ladakh Himalaya, India. Tectonophysics 174, 301-314.

Searle, M., Corfield, R.I., Stephenson, B., McCarron, J., 1997. Structure of the North Indian continental margin in the Ladakh-Zanskar Himalayas: implications for the timing of obduction of the Spontang ophiolite, India-Asia collision and deformation events in the Himalaya. Geological Magazine 134, 297-316.

Searle, M.P., Weinberg, R.F., Dunlap, W.J., 1998. Transpressional tectonics along the Karakoram fault zone, northern Ladakh: constraints on Tibetan extrusion. In: Holdsworth, R.E., Strachan, R.E., Dewey John, F. (Eds.), Continental Transpressional and Transtensional Tectonics. Geological Society, London, pp. 307-326.

Searle, M., Khan, M.A., Fraser, J., Gough, S., Jan, M.Q., 1999. The tectonic evolution of the Kohistan-Karakoram collision belt along the Karakoram Highway transect, north Pakistan. Tectonics 18, 929-949.

Searle, M.P., Warren, C.J., Waters, D.J., Parrish, R.R., 2003. Subduction zone polarity in the Oman Mountains: implications for ophiolite emplacement. Ophiolites in Earth History $218,467-480$.

Seton, M., Müller, R.D., Zahirovic, S., Gaina, C., Torsvik, T., Shephard, G., Talsma, A., Gurnis, M., Turner, M., Maus, S., Chandler, M., 2012. Global continental and ocean basin reconstructions since 200 Ma. Earth-Science Reviews 113, 212-270.

Shellnutt, J.G., Lee, T.Y., Brookfield, M.E., Chung, S.L., 2014. Correlation between magmatism of the Ladakh Batholith and plate convergence rates during the IndiaEurasia collision. Gondwana Research 26, 1051-1059.

Shervais, J.W., 1982. Ti-V plots and the petrogenesis of modern and ophiolitic lavas. Earth and Planetary Science Letters 59, 101-118. 
Sinclair, H.D., Jaffey, N., 2001. Sedimentology of the Indus Group, Ladakh, northern India: implications for the timing of initiation of the palaeo-Indus River. Journal of the Geological Society of London 158, 151-162.

Singh, S., Kumar, R., Barley, M.E., Jain, A.K., 2007. SHRIMP U-Pb ages and depth of emplacement of Ladakh Batholith, Eastern Ladakh, India. Journal of Asian Earth Sciences 30, 490-503.

Stampfli, G.M., Borel, G.D., 2002. A plate tectonic model for the Paleozoic and Mesozoic constrained by dynamic plate boundaries and restored synthetic oceanic isochrons. Earth and Planetary Science Letters 196, 17-33.

Steck, A., 2003. Geology of the NW Indian Himalaya. Eclogae Geologicae Helvetiae 96, 147-U113.

Steck, A., Spring, L., Vannay, J.C., Masson, H., Bucher, H., Stutz, E., Marchant, R., Tieche, J.C., 1993. The tectonic evolution of the northwestern Himalaya in eastern Ladakh and Lahul, India. In: Treloar, J., Searle (Eds.), Himalayan Tectonics. Geological Society of London, London, United Kingdom, pp. 265-276.

Stern, R.J., 2004. Subduction initiation: spontaneous and induced. Earth and Planetary Science Letters 226, 275-292.

Sterne, E.J., 1979. Report on Geological Traverses Across the Indus-Tsangpo Suture Zone in Ladakh, Northern India. Harvard University.

St-Onge, M.R., Rayner, N., Palin, R.M., Searle, M.P., Waters, D.J., 2013. Integrated pressuretemperature-time constraints for the Tso Morari dome (Northwest India): implications for the burial and exhumation path of UHP units in the western Himalaya. Journal of Metamorphic Geology 31, 469-504.

Sun, S.S., McDonough, W.F., 1989. Chemical and isotopic systematics of oceanic basalts: implications for mantle composition and processes. Geological Society, London, Special Publications 42, 313-345.

Tewari, A., 1964. On the Upper Tertiary deposits of Ladakh Himalayas and correlation of various geotectonic units of Ladakh with those of the Kumaon-Tibet Region. Geol. Congr. New Delhi, Sect. II, pp. 37-58.

Thirlwall, M.F., Anczkiewicz, R., 2004. Multidynamic isotope ratio analysis using MC-ICP$\mathrm{MS}$ and the causes of secular drift in $\mathrm{Hf}, \mathrm{Nd}$ and $\mathrm{Pb}$ isotope ratios. International Journal of Mass Spectrometry 235, 59-81.

Vadlamani, R., Guha, D., 2002. Ultrapotassic post-collisional dyke from the Ladakh batholith, Northwest Himalaya. Journal of the Geological Society of India 59, 473-476.

Vervoort, J.D., Patchett, P.J., Söderlund, U., Baker, M., 2004. Isotopic composition of Yb and the determination of Lu concentrations and $\mathrm{Lu} / \mathrm{Hf}$ ratios by isotope dilution using MC-ICPMS. Geochemistry, Geophysics, Geosystems 5.

Wang, X.B., Bao, P.S., Xiao, X.C., 1987. Ophiolites of the Yarlung Zangbo (Tsangbo) River, Xizang (Tibet). Publishing House of Surveying and Mapping (118 pp., plus foldout Geological Map of the Ophioilte Zone along the Middle Yarlung Zangbo (Tsangbo) River, Xizang (Tibet), Beijing).

Wang, C.S., Liu, Z.F., et al., 1999. Xigaze Forearc Basin and Yarlung Zangbo Suture Zone, Tibet. Geological Publishing House, Beijing.

Wang, J.G., Hu, X.M., Garzanti, E., An, W., Liu, X.C., 2017. The birth of the Xigaze forearc basin in southern Tibet. Earth and Planetary Science Letters 465, 38-47.

Weinberg, R.F., Dunlap, W.J., 2000. Growth and Deformation of the Ladakh Batholith, Northwest Himalayas: implications for timing of continental collision and origin of calc-alkaline batholiths. Journal of Geology 108, 303-320.

Whattam, S.A., Stern, R.J., 2011. The 'subduction initiation rule': a key for linking ophiolites, intra-oceanic forearcs, and subduction initiation. Contributions to Mineralogy and Petrology 162, 1031-1045.

White, L.T., Ahmad, T., Ireland, T.R., Lister, G.S., Forster, M.A., 2011. Deconvolving episodic age spectra from zircons of the Ladakh Batholith, northwest Indian Himalaya. Chemical Geology 289, 179-196.

Williams, I.S., 1998. U-Th-Pb geochronology by ion microprobe. Reviews in Economic Geology 7, 1-35.

Winchester, J.A., Floyd, P.A., 1977. Geochemical discrimination of different magma series and their differentiation products using immobile elements. Chemical Geology 20, 325-343.

Wood, D.A., 1980. The application of a ThHfTa diagram to problems of tectonomagmatic classification and to establishing the nature of crustal contamination of basaltic lavas of the British Tertiary Volcanic Province. Earth and Planetary Science Letters 50, 11-30.

Woodhead, J., Hergt, J., Shelley, M., Eggins, S., Kemp, R., 2004. Zircon Hf-isotope analysis with an excimer laser, depth profiling, ablation of complex geometries, and concomitant age estimation. Chemical Geology 209, 121-135.

Xia, B., Yu, H.X., Chen, G.W., Qi, L., Zhao, T.P., Zhou, M.F., 2003. Geochemistry and tectonic environment of the Dagzhuka ophiolite in the Yarlung-Zangbo suture zone, Tibet. Geochemical Journal 37, 311-324.

Yang, T.S., Ma, Y.M., Zhang, S.H., Bian, W.W., Yang, Z.Y., Wu, H.C., Li, H.Y., Chen, W.W., Ding, J.K., 2015. New insights into the India-Asia collision process from Cretaceous paleomagnetic and geochronologic results in the Lhasa terrane. Gondwana Research 28, 625-641.

Zahirovic, S., Muller, R.D., Seton, M., Flament, N., Gurnis, M., Whittaker, J., 2012. Insights on the kinematics of the India-Eurasia collision from global geodynamic models. Geochemistry, Geophysics, Geosystems 13.

Zaigham, N.A., Mallick, K.A., 2000. Bela ophiolite zone of southern Pakistan: tectonic setting and associated mineral deposits. Geological Society of America Bulletin 112, 478-489.

Zhou, M.F., Robinson, P.T., Malpas, J., Li, Z.J., 1996. Podiform chromitites in the Luobusa ophiolite (southern Tibet): implications for melt-rock interaction and chromite segregation in the upper mantle. Journal of Petrology 37, 3-21.

Zhou, Y.N., Cheng, X., Yu, L., Yang, X.F., Su, H.L., Peng, X.M., Xue, Y.K., Li, Y.Y., Ye, Y.K., Zhang, J., Li, Y.Y., Wu, H.N., 2016. Paleomagnetic study on the Triassic rocks from the Lhasa Terrane, Tibet, and its paleogeographic implications. Journal of Asian Earth Sciences 121, 108-119.

Zyabrev, S.V., Kojima, S., Ahmad, T., 2008. Radiolarian biostratigraphic constraints on the generation of the Nidar ophiolite and the onset of Dras arc volcanism: tracing the evolution of the closing Tethys along the Indus-Yarlung-Tsangpo suture. Stratigraphy 5, 99-112.

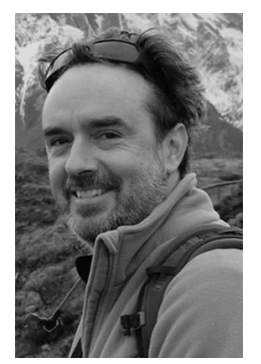

Solomon Buckman is a Senior Lecturer in economic and field geology at the School of Earth and Environmental Sciences at the University of Wollongong in Australia. Prior to that he was a geology lecturer at the University of South Australia. He undertook a post-doc position at James Cook University investigating the Cannington $\mathrm{Ag}-\mathrm{Pb}-\mathrm{Zn}$ deposit after completing his PhD at the University of Hong Kong in 2000, which was a study of the tectonic evolution of West Junggar within the Central Asian Orogenic Belt of NW China. Solomon worked as an exploration geologist across Australia for two years after completing his B.Sc. (Hons) at the University of Sydney in 1993. He is a field geologist with interests and expertise in the tectonic evolution of ophiolites and island arcs in eastern Australia (New England and Lachlan orogens), the Himalayas, China and South America. His interests are in convergent margin tectonics and in particular, mechanisms of continental growth involving the addition of juvenile, oceanic terranes to continental margins.

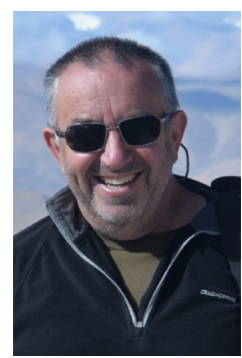

Jonathan C. Aitchison is Professor and Head of the School of Earth and Environmental Sciences at The University of Queensland, Australia. Prior to that he was at The University of Sydney and the University of Hong Kong. He received his $\mathrm{PhD}$ from the University of New England, NSW Australia in 1989 and studied for his BSc (Hons) and MSc at the University of Otago in New Zealand and studied in Japan at Niigata and Kochi universities. His research interests include the evolution of convergent plate margins and collisional orogens especially arc-continent collisions worldwide. In particular, he has concentrated on the SW Pacific, Japan, eastern Australia, New Caledonia and the Philippines. His primary interest is the India-Asia collision particularly in Tibet, where he has worked for two decades. As an additional research theme, he also works on radiolarians to provide the biostratigraphic age constraints necessary for unravelling wicked tectonic problems.

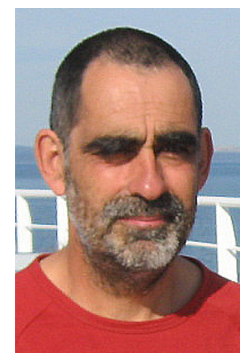

Allen Nutman is a professor and current head of school at the University of Wollongong. He works mostly on early Precambrian basement rocks of Greenland, Australia, China, Brazi and Russia with occasional excursions into the Phanerozoic orogens of eastern Australia. He integrates his own mapping and field observations with U-Pb zircon geochronology. He has 25 years of experience working on SHRIMP ion microprobe $\mathrm{U}-\mathrm{Pb}$ geochronology on 8 different instruments (I, IIs and RG) in Australia, China, Japan and Korea. His research interests include evolution of the Eoarchaean Earth and the appearance of earliest life.

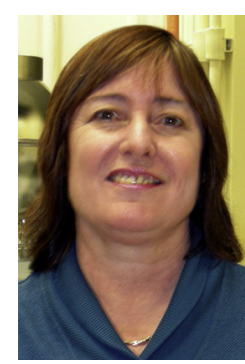

Vickie Bennett is a professor and associate director at the Research School of Earth Sciences, the Australian National University, Canberra, Australia and head of the SPIDE $_{2} \mathrm{R}$ isotope laboratory. Her research focuses on the development and application of radiogenic isotopic methods to understanding the origin and evolution of Earth's continental crust and mantle reservoirs, early planetary differentiation, and geospherebiosphere interactions in deep time. She is co-editor of the 1 st (2007) and 2ed (2018) editions of the major reference book "Earth's Oldest Rocks". Bennett is a Geochemical Fellow and a Fellow of the Geological Society of America and is currently Vice-President (President-elect) of the Geochemical Society.

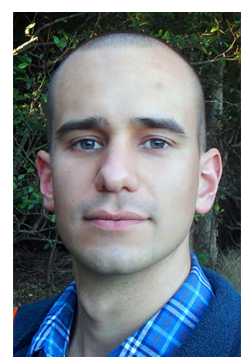

Wanchese M. Saktura is a PhD candidate at University of Wollongong, Australia. He obtained B.Sc. (Adv) Honours 1st Class for his research on tectonic evolution of the Beishan Orogen, China. His current research is focused on the Himalayan Orogeny, more precisely, tectonic accretionary processes and paleogeography along Tethyan oceans prior to the final continentcontinent collision. Additional interests include application of thermochronology in orogenic settings, especially, use of optically stimulated luminescence (OSL) technique in geology to understand most recent neotectonic processes and evolution of mountainous relief. 


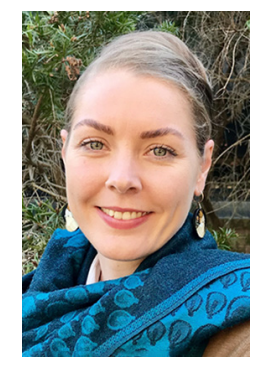

Jessica Walsh completed a Bachelor of Science (Honours) at the University of Wollongong (UOW), Australia in 2015, which focused on multiple magmatic related Mo-Cu systems in the Qinling Orogenic Belt, Central China. She is currently completing her $\mathrm{PhD}$ at the same institute, with her research focusing on the geotectonic evolution of the Himalaya, with specific reference to arc-continent collision prior to final continentcontinent collision of India and Eurasia. Broadly, her research interests include tectonic evolution of orogenic belts and continental growth processes.

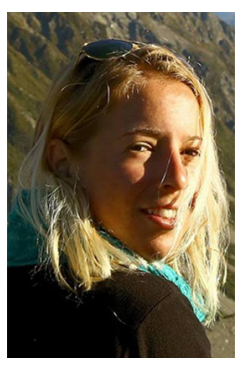

Sarah Kachovich is a $\mathrm{PhD}$ candidate at The University of Queensland. She specialises in radiolarian biostratigraphy, with her research focusing on linking microfossils to tectonic problems, such as the Himalayan collision and the tectonic evolution of the New England Orogen, eastern Australia.

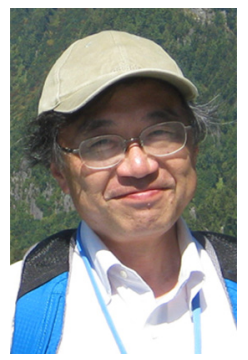

Hiroshi Hidaka is a foundation researcher of the Hiroshima SHRIMP group - the first SHRIMP large ion microprobe instrument to be sold outside of Australia and which was installed in 1997. He is an isotope geochemist with a wide range of interests, both from the perspective of geology and also nuclear science. Concerning the latter, he has worked extensively on the natural (Palaeoproterozoic) nuclear reactor from Oklo in West Africa. In this, his studies involve both the use of conventional IDTIMS methods and the use of SHRIMP to document the distribution of fission products on the mineral-grain scale. From the geological perspective he has undertaken extensive U-Pb zircon dating using SHRIMP, both on Japanese projects and in collaboration with overseas researchers. One overseas link has been with co-author on this paper Allen Nutman, mostly investigating early crustal evolution via ancient rocks in Greenland. Prof Hidaka is currently a faculty member at Nagoya University. 